K. Yamato

Nagoya Math. J.

Vol. 49 (1973), 155-229

\title{
QUALITATIVE THEORY OF CODIMENSION-ONE FOLIATIONS
}

\author{
KAZUO YAMATO
}

Introduction. The object of the present paper is to give a method of studying the topological properties of integral manifolds defined by a completely integrable one-form.

Our method is differential-topological. Through the singular points of the variation equation of the given one-form, we investigate the qualitative properties of the integral manifolds.

The plan of this paper is as follows. In $\S 1$, we state our main theorems, which assert that under certain conditions, foliated structures are classified into three groups, "bundle foliations", "Reeb foliations", "hyperbolic foliations". In $\S 2$, which is one of the most important parts in our theory, we introduce the concept of a vein. A vein is a leaf of a certain codimension-two foliation associated with the given foliation. Proposition 2.2.1 is concerned with the existence of compact veins. In $\S 3$, we study precisely the distance between two leaves along a curve contained in one of them. "Admissible tangential curves" and their "lifts" are the fundamental tools in the proofs of the main theorems. In $\S 4$, we introduce a special Riemannian structure convenient for the proof of Proposition 4.2.1 from which we prove three fundamental lemmas 4.1.2-4.1.4. Using these lemmas, we prove our theorems I, II, III, in $\S \S 5,6,7$, respectively. $\S 8$ is devoted to the proofs of Propositions 4.1.1 and 4.2.1. In the appendix, we prove that our condition (T) is "generic".

The main results of this paper have been announced in [8].

The author wishes to express his gratitude for the guidance and encouragement received from Professor Y. Shikata.

Notation. $\boldsymbol{R}$ denotes the field of real numbers, and $\boldsymbol{R}^{m}$ denotes the real $m$-space, regarded as a real vector space or as a smooth manifold. $S^{m}$ denotes an ordinary $m$-dimensional sphere. By an $m$-manifold, we

Received September 28, 1972. 
mean an $m$-dimensional manifold with or without boundary, of class $C^{3}$. By a closed $m$-manifold, we mean a compact $m$-manifold without boundary. All functions, maps, curves, and vector fields are assumed to be of class $C^{1}$ unless otherwise stated. All Riemannian structures are assumed to be of class $C^{2}$. We denote by $\mathscr{L}_{X}$ the Lie derivative with respect to a vector field $X$. A one-form $\alpha$ on a manifold $M$ is to be understood a map of the tangent bundle $T(M)$ into $R$, which induces, at each point $x$, a linear map $\alpha_{x}$ of the tangent space $T_{x}(M)$ at $x$ into $\boldsymbol{R}$. For two subsets $A, B$ of a Riemannian manifold, we shall write dis $(A, B)$ for the distance of $A$ and $B$, and $\operatorname{diam}(A)$ for the diameter of $A$. For a map $f$ of a manifold $M$ into another manifold $M^{\prime}$, we write $f_{*}$ for the bundle map of $T(M)$ into $T\left(M^{\prime}\right)$ induced by $f$. For a curve $c$ on a manifold, we denote by $\dot{c}(t)$ the tangent vector at a point $c(t)$. If a curve $c$ is defined on an interval $[a, b]$, we write $\int_{c[a, u]} \alpha$ for the integral of $\alpha$ over $[a, u] \subset[a, b]$, i.e.,

$$
\int_{c[a, u]} \alpha=\int_{a}^{u} \alpha(\dot{c}(t)) d t
$$

For a piecewise $C^{1}$ curve, the same integral as above can be defined. We write $(M, \alpha)$ for a manifold $M$ with a specified one-form $\alpha$, and $(M$, $\alpha, \mathfrak{g})$ for $(M, \alpha)$ with a specified Riemannian structure $\mathfrak{g}$. We shall say that $(M, \alpha)$ is a foliated manifold if $\alpha$ is completely integrable, i.e., $\alpha \wedge d \alpha=0$. If $\alpha$ is nonsingular, a maximal connected integral manifold of a foliated manifold $(M, \alpha)$ will be called a leaf (of $(M, \alpha)$ or of $M$ ). Given a foliated manifold $(M, \alpha)$ by "almost every leaf", we mean except for leaves whose union has measure zero in $M$. It is clear that for a finite set $A$ in a foliated manifold, almost every leaf does not intersect $A$.

\section{§1. Statement of the main theorems}

Suppose that we are given a connected, closed $(n+1)$-manifold $V^{n+1}$, $n \geq 1$, of class $C^{r+1}$ with a nonsingular, completely integrable one-form $\omega$ of class $C^{r}$. Throughout this paper, we denote by $(V, \omega)$ this foliated manifold and assume, for simplicity, that $r$ is sufficiently large, e.g., $r \geq 21$. (From the proofs, one will see that our main theorems hold if $r \geq 4$.)

1.1. The critical cycle $\Sigma$. Since $\omega$ is nonsingular and completely 
integrable, it is well known that for each point $p$ of $V$ there is a local coordinate system $\left(x^{1}, \cdots, x^{n+1}\right)$ of class $C^{r}$ in a neighborhood $U$ of $p$ such that $\omega \mid U=f d x^{n+1}$ for some positive-valued $C^{r-1}$ function $f$ defined on $U$. Then the set $\left(U, f ; x^{1}, \cdots, x^{n+1}\right)$ is called an $\mathscr{F}$-chart $(a t p)$. Now, let $\Sigma$ be the set of zeros of the exterior derivative of $\omega$, i.e.,

$$
\Sigma=\left\{p \in V \mid(d \omega)_{p}=0\right\} .
$$

For a point $p$ of $\Sigma$, we define the type of $p$ as follows. Let $\left(U, f ; x^{1}\right.$, $\cdots, x^{n+1}$ ) be an $\mathscr{F}$-chart at $p$, and consider two matrices

$$
\begin{aligned}
\left\|\partial^{2} f(x)\right\| & =\left(f_{i j}(x) ; \begin{array}{l}
i \downarrow 1, \cdots, n \\
j \rightarrow 1, \cdots, n
\end{array}\right), \\
\left\|\partial^{3} f(x)\right\| & =\left(f_{i j}(x), \frac{\partial}{\partial x^{i}} \operatorname{det}\left\|\partial^{2} f(x)\right\| ; \begin{array}{l}
i \downarrow 1, \cdots, n+1 \\
j \rightarrow 1, \cdots, n
\end{array}\right),
\end{aligned}
$$

where $f_{i j}(x)=\partial^{2} f(x) / \partial x^{i} \partial x^{j}$.

Let $\lambda=0,1, \cdots, n$. The point $p$ is said to be of type $(\lambda)$ if the matrix $\left\|\partial^{2} f(p)\right\|$ is nonsingular and if the number of negative eigenvalues of $\left\|\partial^{2} f(p)\right\|$ is equal to $\lambda$. We say that $p$ is of type $(*)$ if $\left\|\partial^{2} f(p)\right\|$ is singular. It is obvious that the type of a point of $\Sigma$ is well defined independently of the choice of $\mathscr{F}$-charts. For $t=0,1, \ldots, n$ or $*$, let $\Sigma_{t}$ be the set of points of type $(t)$. Then we have

$$
\Sigma=\Sigma_{*} \cup \Sigma_{0} \cup \Sigma_{1} \cup \cdots \cup \Sigma_{n} \text { (disjoint union). }
$$

We shall assume that $\omega$ satisfies the following condition:

For any point $p$ of $\Sigma_{*}$, there is an $\mathscr{F}$-chart $\left(U, f ; x^{1}, \cdots, x^{n+1}\right)$ at $p$ such that $\left\|\partial^{3} f(p)\right\|$ is nonsingular.

One sees then that the same condition holds for any $\mathscr{F}$-chart at $p \in \Sigma_{*}$. One sees also that Condition (T) implies that $\Sigma$ is a closed one-manifold. Hence if $\omega$ satisfies (T), then $\Sigma$ will be called the critical cycle (of ( $V$, $\omega)$ ). In the appendix, it will be proved that Condition ( $\mathrm{T}$ ) is generic.

1.2. The main theorems. Assume that $\omega$ satisfies Condition ( $T$ ). Then we have the following three theorems.

THEOREM I. If $\Sigma_{0} \neq \emptyset$ and $\Sigma_{1}=\emptyset$, then there exists a $C^{r}$ fibre bundle $B^{n+1}$ over $S^{1}$ and a $C^{r}$ diffeomorphism $h: B^{n+1} \rightarrow V^{n+1}$ such that

(i) the fibre of $B^{n+1}$ is a connected, simply connected, closed $n$ manifold of class $C^{r}$. 
(ii) for each fibre $M^{n}$ of $B^{n+1}$, the diffeomorphism $h$ induces a $C^{r}$ diffeomorphism of $M^{n}$ onto a leaf of $V$.

THEOREM II. If $\Sigma_{n} \neq \emptyset, \Sigma_{n-1}=\emptyset$, and $\Sigma_{0}=\emptyset$, then for any point $p$ of $\Sigma_{n}$, there exists a $C^{r}$ fibre bundle $R^{n+1}$ over $S^{1}$, and a $C^{r}$ imbedding $h: R^{n+1} \rightarrow V^{n+1}$ such that

(i) the fibre of $R^{n+1}$ is a connected, simply connected, noncompact n-manifold without boundary.

(ii) for each fibre $N^{n}$ of $R^{n+1}$, the imbedding $h$ induces a $C^{r}$ diffeomorphism of $N^{n}$ onto a leaf of $V$.

(iii) the set $h\left(R^{n+1}\right) \cap \Sigma_{n}$ coincides with the connected component of $\Sigma$ containing $p$.

(iv) if $\mathrm{Cl}\left(\Sigma_{1} \cap h\left(R^{n+1}\right)\right) \subset h\left(R^{n+1}\right)$, then there exist a finite number of compact leaves $K_{1}, \cdots, K_{m}$ such that

$$
K_{1} \cup \cdots \cup K_{m}=\mathrm{Cl} h\left(R^{n+1}\right)-h\left(R^{n+1}\right)=\mathrm{Cl} L-L
$$

for any leaf $L$ in $h\left(R^{n+1}\right)$.

THEOREM III. If $\Sigma_{n}=\emptyset$, then there exists an open, dense subset $V_{0}$ of $V$ such that for any $p \in V_{0}$, the leaf through $p$ is locally dense in the sense of Reeb (see 7.1).

\section{§. The veined structure}

2.1. The veined structure. Let $X$ be a vector field on $V$ such that $\omega(X)=1$, and put $\omega^{\prime}=-\mathscr{L}_{X} \omega$.

Lemma 2.1.1. For an $\mathscr{F}$-chart $\left(U, f ; x^{1}, \ldots, x^{n+1}\right)$, we have

$$
\omega^{\prime} \mid U=\sum_{i=1}^{n}\left(\partial \log f / \partial x^{i}\right) d x^{i}+\left(-X(f)+\partial \log f / \partial x^{n+1}\right) d x^{n+1} .
$$

Proof. This is an easy consequence of the following elementary formulas:

$$
\begin{gathered}
\left(\mathscr{L}_{X} \omega\right)(Y)=X(\omega(Y))-\omega([X, Y]), \\
{[g Y, h Z]=g h[Y, Z]+g(Y h) Z-h(Z g) Y}
\end{gathered}
$$

for vector fields $Y, Z$ and functions $g, h$ on $V$, where [,] denotes the bracket.

By this lemma, we know that the map $\omega^{\prime} \mid \omega^{-1}(0): \omega^{-1}(0) \rightarrow \boldsymbol{R}$ is defined independently of the choice of $X$, where $\omega^{-1}(0)$ denotes the subbundle of 
$T(V)$ defined by $\omega=0$. Furthermore, we see that the one-form $\omega^{\prime} \mid L$ on each leaf $L$ is closed. Hence, the following definition makes sense.

DEFINITION 2.1.1. The veined structure of the foliated manifold $(V, \omega)$ is the "codimension-two" foliation on $V$ defined by $\omega=\omega^{\prime}=0$. The leaves of this "codimension-two" foliation will be called the veins of $(V, \omega)$.

More precisely, a vein of $(V, \omega)$ is a nonempty subset $J$ in $V$ having the following properties:

(i) any two points $x, y \in J$ can be joined by a piecewise $C^{1}$ curve $c:[0, \tau] \rightarrow V$ such that $\omega(\dot{c}(t))=\omega^{\prime}(\dot{c}(t))=0$ for all $t \in[0, \tau]$ at which $c$ is differentiable ;

(ii) any point $x \in V$ which can be joined to a point $\in J$ by a piecewise $C^{1}$ curve $c$ satisfying the same condition as in (i), belongs to $J$.

A vein $J$ will be said to be nonsingular if $J$ does not contain any singular point of $\omega^{\prime} \mid \omega^{-1}(0)$, i.e., any point $x \in V$ such that $\omega_{x}^{\prime}=0$ on $\omega_{x}^{-1}(0)$. Clearly, a nonsingular vein is a connected $(n-1)$-submanifoldwithout-boundary (not necessarily closed) in $V$, of class $C^{r}$.

2.2. Closed one-forms and Morse theory. Let $M^{n}$ be a connected, complete Riemannian $n$-manifold of class $C^{3}$, without boundary. Let $\alpha$ be a closed one-form of class $C^{2}$. Denote by $\alpha^{*}$ the dual vector field of $\alpha$ and denote by \|\| the norm of tangent vectors or cotangent vectors of $M$. For a singular point $p$ of $\alpha$, the index of $p$ is defined to be the number of negative eigenvalues of the Jacobian matrix of $\alpha$ at $p$. The one-form $\alpha$ is said to be proper if every singular point is nondegenerate and the vector field $\alpha^{*}$ is complete, and if there exist two families $\left\{E_{i}\right\}_{i \in I},\left\{\tilde{E}_{i}\right\}_{i \in I}$ of open sets of $M$ satisfying the following conditions:

(i ) $E_{i} \subset \tilde{E}_{i}$ for each $i \in I$, and $\tilde{E}_{i} \cap \tilde{E}_{j}=\emptyset$ for every $i, j, i \neq j$;

(ii) for each singular point $p$ of $\alpha$, there is $i \in I$ such that $p \in E_{i} \subset \tilde{E}_{i}$;

(iii) there exist three positive constants $a_{0}, b_{0}, c_{0}$ such that (a) $\left\|\alpha_{x}\right\|>a_{0}$ for all $x \in M-\cup_{i \in I} E_{i}$, (b) $\operatorname{dis}\left(E_{i}, M-\tilde{E}_{i}\right)>b_{0}$ for all $i \in I$, and (c) $\operatorname{diam}\left(\tilde{E}_{i}\right)<c_{0}$ for all $i \in I$.

Proposition 2.2.1. Suppose that $\alpha$ is proper and has at least one singular point of index 0 . If $\alpha$ has no singular point of index 1 , then the following hold:

(i) There exists a $C^{3}$ function $f: M \rightarrow \boldsymbol{R}$ which is proper, i.e., every 
inverse image of compact sets is compact, such that $\alpha=d f$.

(ii) $M^{n}$ is simply connected.

(iii) If $\alpha$ has no singular point of index $n$, then $M^{n}$ is noncompact.

(iv) The number of the singular points of index 0 , is equal to one.

(v) For any $y \in M$, there exists a piecewise $C^{1}$ curve $\mathfrak{a}:[0, \tau] \rightarrow M$ such that $\mathfrak{a}(\tau)=y$ and $\mathfrak{a}(0)$ is the singular point of index 0 , and such that for any $x=\mathfrak{a}(t)$ at which the vector field $\alpha^{*}$ does not vanish, the tangent vector $\dot{\mathfrak{a}}(t)$ coincides with $\alpha_{x}^{*} /\left\|\alpha_{x}^{*}\right\|$.

This will be proved in 2.3. The following proposition can be proved similarly (cf. [9]).

Proposition 2.2.2. Suppose that $\alpha$ is proper. Suppose that there exists a closed $(n-1)$-manifold $J^{n-1}$ and an immersion $i: J \rightarrow M$ such that for any $x \in J$, the subspace $i_{*}\left(T_{x}(J)\right)$ of $T_{i(x)}(M)$ does not contain the vector $\alpha_{i(x)}^{*}$, and such that the cohomology class $\left[i^{*} \alpha\right] \in H^{1}(J ; R)$ is trivial, where $i_{*}, i^{*}$ are the induced maps. If $\alpha$ has no singular point of index 1 and no singular point of index $n-1$, then $\alpha$ is rational, i.e., for any $x \in M$, there exists an open subset $U$ of $M$ containing $x$ and a function. $f: U \rightarrow \boldsymbol{R}$ of class $C^{3}$ such that $\alpha=d f$ on $U$ and each level manifold $f^{-1}(h)$, $h \in \operatorname{Im} f$, is compact.

Remark 2.2.1. If $V$ is Riemannian and if $\omega$ satisfies Condition (T), then for any leaf $L$ of $(V, \omega)$ which does not contain any point of $\Sigma_{*}$, hence for almost every leaf $L$, the closed one-forms $\pm \omega^{\prime} \mid L$ on the manifold $L$ with the induced Riemannian structure, are proper in our sense. This may be seen as follows. Condition (T) implies that the critical cycle $\Sigma$ is a closed one-manifold $S^{1} \cup \cdots \cup S^{1}$ and is tangent to the leaves, at the finite set $\Sigma_{*}$. For each $p \in \Sigma_{*}$, choose an $\mathscr{F}$-chart $\left(U, f ; x^{1}, \cdots\right.$, $\left.x^{n+1}\right)$ at $p$ such that $x^{1}(p)=\cdots=x^{n+1}(p)=0$ and put

$$
\begin{aligned}
& U(p)=\left\{q \in U|| x^{i}(q) \mid<\varepsilon / 2, i=1, \cdots, n+1\right\}, \\
& \tilde{U}(p)=\left\{q \in U|| x^{i}(q)|<\varepsilon, i=1, \cdots, n ;| x^{n+1}(q) \mid<\varepsilon / 2\right\},
\end{aligned}
$$

where $\varepsilon$ is a small positive number such that $U(p), \tilde{U}(p)$ are homeomorphic to the open sets

$$
\begin{aligned}
& \left\{x \in \boldsymbol{R}^{n+1}|| x^{i} \mid<\varepsilon / 2, i=1, \cdots, n+1\right\}, \\
& \left\{x \in \boldsymbol{R}^{n+1}|| x^{i}|<\varepsilon, i=1, \cdots, n ;| x^{n+1} \mid<\varepsilon / 2\right\},
\end{aligned}
$$

by $\varphi=\left(x^{1}, \cdots, x^{n+1}\right)$, respectively. Let $T, \tilde{T}$ be two sufficiently small 
tubular neighborhoods of $\Sigma$ in $V$ such that $\mathrm{Cl} T \subset \tilde{T}$, and put

$$
E=U\left(p_{1}\right) \cup \cdots \cup U\left(p_{s}\right) \cup T, \quad \tilde{E}=\tilde{U}\left(p_{1}\right) \cup \cdots \cup \tilde{U}\left(p_{s}\right) \cup \tilde{T},
$$

where $\left\{p_{1}, \cdots, p_{s}\right\}=\Sigma_{*}$. Then, for a leaf $L$ which does not contain any point of $\Sigma_{*}$, it is easily verified that the forms $\pm \omega^{\prime} \mid L$ on $L$ have only nondegenerate singular points (by Lemma 2.1.1.) and are proper, with the families $\left\{E_{i}\right\}_{i \in I},\left\{\tilde{E}_{i}\right\}_{i \in I}$ of open sets $E_{i}, \tilde{E}_{i}$ defined by the following conditions: $\bigcup_{i \in I} E_{i}=L \cap E, \cup_{i \in I} \tilde{E}_{i}=L \cap \tilde{E}$ (disjoint unions), and $E_{i}$, $\tilde{E}_{i}$ are connected components of $L \cap E, L \cap \tilde{E}$, respectively.

2.3. Proof of Proposition 2.2.1. We begin with some definitions. For a $C^{1}$ curve $c:[a, b] \rightarrow M$, the integral of $\alpha$ on $c$ is defined to be $\int_{a}^{b} \alpha(\dot{c}(t)) d t$ and will be denoted by $\int_{c(t), a \leq t \leq b} \alpha$. A compact leaf of $(M, \alpha)$ is defined to be a nonempty, arcwise connected, compact subset $J$ in $M$ satisfying the following condition: for each $x \in J$, there exists a neighborhood $U$ of $x$ in $M$ and a function $f: U \rightarrow R$ such that $\alpha=d f$ on $U$ and $J \cap U=$ $f^{-1}(f(x))$. A compact leaf $J$ is said to be singular if $J$ contains any singular points of $\alpha$. Clearly, a nonsingular, compact leaf is a connected, closed $(n-1)$-submanifold in $M$. Let $\left\{\psi_{t}\right\}$ be the one-parameter group of transformations generated by $\alpha^{*}$. The proof of Proposition 2.2.1 will be preceded by six lemmas, 2.3.1-2.3.6.

2.3.1. There exist positive constants $d_{1}, h_{1}$ satisfying the following condition: for $x \in M$ and $\tau>0$, if $\operatorname{dis}\left(x, \psi_{\tau}(x)\right)>d_{1}$, then the integral of $\alpha$ on the curve $\psi_{t}(x), 0 \leq t \leq \tau$, is greater than $h_{1}$.

Proof. Let $\left\{E_{i}\right\},\left\{\tilde{E}_{i}\right\}, a_{0}, b_{0}, c_{0}$ be as in the definition of "proper". Put $d_{1}=\max \left(b_{0}, c_{0}\right), h_{1}=a_{0} b_{0}$, and let the curve $\psi_{t}(x), 0 \leq t \leq \tau$, satisfy $\operatorname{dis}\left(x, \psi_{\tau}(x)\right)>d_{1}$. First, consider the case where the curve $\psi_{t}(x), 0 \leq t \leq \tau$, does not intersect $E_{i}$ for any $i \in I$. Then, $\left\|\alpha_{\psi_{t}(x)}^{*}\right\|=\left\|\alpha_{\psi_{t}(x)}\right\|>a_{0}$, hence we have

$$
\begin{aligned}
\int_{\psi_{t}(x), 0 \leq t \leq \tau} \alpha & =\int_{0}^{\tau} \alpha\left(\alpha_{\psi_{t}(x)}^{*}\right) d t=\int_{0}^{\tau}\left\|\alpha_{\psi_{t}(x)}^{*}\right\|^{2} d t \\
& >a_{0} \int_{0}^{\tau}\left\|\alpha_{\psi_{t}(x)}^{*}\right\| d t \geq a_{0} \operatorname{dis}\left(x, \psi_{\tau}(x)\right)>a_{0} d_{1} \geq h_{1} .
\end{aligned}
$$

Next, in the case where the curve $\psi_{t}(x), 0 \leq t \leq \tau$, intersects some $E_{i}$, since $\operatorname{dis}\left(x, \psi_{\tau}(x)\right)>d_{1}$, dis $\left(E_{i}, M-\tilde{E}_{i}\right)>b_{0}$, and $\operatorname{diam}\left(\tilde{E}_{i}\right)<c_{0}$, we see 
that there exist real numbers $t_{1}, t_{2}, 0 \leq t_{1}<t_{2} \leq \tau$, such that the curve $\psi_{t}(x), t_{1}<t<t_{2}$, is contained in $\tilde{E}_{i}-E_{i}$, and such that the length of $\psi_{t}(x), t_{1} \leq t \leq t_{2}$, is greater than $b_{0}$. Since $\left\|\alpha_{\psi_{t}(x)}^{*}\right\|>a_{0}$ for $t \in\left[t_{1}, t_{2}\right]$, similarly to the preceding case, we have

$$
\int_{\psi_{t}(x), t_{1} \leq t \leq t_{2}} \alpha>a_{0} b_{0}=h_{1} .
$$

Hence, we obtain

$$
\int_{\psi_{t}(x), 0 \leq t \leq \tau} \alpha \geq \int_{\psi_{t}(x), t_{1} \leq t \leq t_{2}} \alpha>h_{1},
$$

which proves 2.3.1.

2.3.2. Suppose that there exists a connected, compact n-submanifold $W^{n}$ in $M^{n}$ such that the boundary $\partial W$ is the finite union of nonsingular, compact leaves of $(M, \alpha)$, and such that for any $x \in \partial W$, the vector $\alpha_{x}^{*}$ is directed toward the outside of $W$. Suppose further that there exists a function $f: W \rightarrow \boldsymbol{R}$ such that $\alpha=d f$ on $W$. Let $J$ be a connected component of $\partial W$, and let $\delta$ be a positive constant satisfying the following condition: for each $x \in J$, there is a positive number $\tau(x)$ such that the integral of $\alpha$ on the curve $\psi_{t}(x), 0 \leq t \leq \tau(x)$, is equal to $\delta$. Then, the set $\tilde{W}=W \cup\left\{\psi_{t}(x) \mid x \in J, 0 \leq t \leq \tau(x)\right\}$ is a connected, compact $n$-submanifold in $M$ such that the boundary $\partial \tilde{W}$ is the finite union of nonsingular, compact leaves of $(M, \alpha)$, and such that for any $x \in \tilde{W}$, the vector $\alpha_{x}^{*}$ is directed toward the outside of $\tilde{W}$. Furthermore, there exists a function $\tilde{f}: \tilde{W} \rightarrow \boldsymbol{R}$ such that $f=\tilde{f}$ on $W$ and $\alpha=d \tilde{f}$ on $\tilde{W}$.

This is easily verified.

2.3.3. Let $p$ be a nonsingular point of $\alpha$. Suppose that there is a positive number $\delta_{0}$ having the following properties:

(i) for any $\delta \in\left(0, \delta_{0}\right)$, there is a positive number $\tau(\delta)$ such that the integral of $\alpha$ on the curve $\psi_{t}(p), 0 \leq t \leq \tau(\delta)$, is equal to $\delta$;

(ii) for any $\tau>0$, the integral of $\alpha$ on the curve $\psi_{t}(p), 0 \leq t \leq \tau$, is smaller than $\delta_{0}$.

Then $\lim _{t \rightarrow \infty} \psi_{t}(p)$ exists and is a singular point of $\alpha$.

Proof. Using 2.3.1, we know that the subset $\cup_{t \geq 0} \psi_{t}(p)$ is bounded in $M$. In fact, this set is contained in the compact set

$$
\left\{x \in M \mid \operatorname{dis}(p, x) \leq d_{1}\left(\delta_{0} h_{1}^{-1}+1\right)\right\},
$$


where $d_{1}, h_{1}$ are the same constants as in 2.3.1. Therefore, since $M$ is complete, there is an infinite sequence $t_{1}<t_{2}<\cdots, t_{i} \rightarrow \infty$, such that $\lim _{i \rightarrow \infty} \psi_{t_{i}}(p)$ exists in $M$. It is easy to verify that the limit point is a singular point of $\alpha$. Since every singular point of $\alpha$ is nondegenerate, the limit point is uniquely determined. This proves 2.3.3.

2.3.4. Suppose that there is a nonsingular, compact leaf $J$ of $(M, \alpha)$. Let $\delta_{0}$ be a positive number satisfying the following condition: for any $\delta \in\left(0, \delta_{0}\right)$ and any $x \in J$, there exists a positive number $\tau(x, \delta)$ such that the integral of $\alpha$ on $\psi_{t}(x), 0 \leq t \leq \tau(x, \delta)$, is equal to $\delta$. Let $S$ be the subset of $J$ consisting of those points $x$ such that for any $\tau>0$, the integral of $\alpha$ on $\psi_{t}(x), 0 \leq t \leq \tau$, is smaller than $\delta_{0}$. If $S \neq \emptyset$, then $S_{0}=\left\{\lim _{t \rightarrow \infty} \psi_{t}(p) \mid p \in S\right\}$ is a finite set, whose elements are singular points of $\alpha$.

Proof. By 2.3.3, for each $p \in S$, the limit point of $\psi_{t}(p)(t \rightarrow \infty)$ exists and is singular. Similarly to the proof of 2.3.3, using 2.3.1, we see that the set $S_{0}$ is bounded in $M$. Since $M$ is complete, and every singular point of $\alpha$ is nondegenerate, we can conclude that $S_{0}$ is finite.

2.3.5. Under the same hypotheses and notations as in 2.3.4, if $S \neq \emptyset$ and if $S_{0}$ contains no singular point of index 1 , then the set

$$
\tilde{J}=S_{0} \cup\left\{\psi_{\tau(x)}(x) \mid x \in J-S\right\},
$$

where $\tau(x)$ is a positive number satisfying $\int_{\psi_{t}(x), 0 \leq t \leq \tau(x)} \alpha=\delta_{0}$, is a singular, compact leaf of $(M, \alpha)$.

This is proved in [9].

2.3.6. Let a singular, compact leaf $J$ of $(M, \alpha)$ which contains no singular point of index 1, be given. Then, there exists a connected, compact $n$-submanifold $W^{n}$ in $M^{n}$ containing $J$ and there exists a function $f: W \rightarrow \boldsymbol{R}$ satisfying $\alpha=d f$ on $W$, such that the boundary $\partial W$ is the finite union of nonsingular, compact leaves of $(M, \alpha)$, and such that the subset $\partial_{-} W$ of $\partial W$ consisting of those points $x$ at which the vector $\alpha_{x}^{*}$ is directed toward the inside of $W$, is connected (therefore $\partial_{-} W$ is a nonsingular, compact leaf if $\partial_{-} W \neq \emptyset$ ).

This is also proved in [9].

Proof of Proposition 2.2.1. Fix a singular point $p$ of index 0. By 
Morse's lemma, there exists an imbedded $n$-disk $D^{n}$ in $M^{n}$ containing $p$ such that the boundary is a leaf of $(M, \alpha)$, and there exists a function $f: D^{n} \rightarrow \boldsymbol{R}$ such that $\alpha=d f$ on $D$. Using this fact and 2.3.1, and applying $2.3 .2,2.3 .5$, and 2.3 .6 , we can easily construct a function $f: M \rightarrow \boldsymbol{R}$ of class $C^{3}$ which is proper and satisnies $\alpha=d f$. Applying Morse theory to the proper function $f: M \rightarrow \boldsymbol{R}$, we see that $M$ is simply connected and the number of the singular points of index 0 is equal to one. Part (iii) is obvious. In order to prove (v), it suffices to verify the following.

2.3.7. Let $U$ be an open set in $\boldsymbol{R}^{n}$ with a Riemannian structure $\mathfrak{g}$ of class $C^{1}$, and let $f: U \rightarrow \boldsymbol{R}$ be a function of class $C^{2}$ with a nondegenerate critical point $p$. Denote by grad $f$ the gradient of $f$ with respect to g. Suppose that there is an integral curve $c:[0, \infty) \rightarrow U$ of grad $f$ such that $c(t) \rightarrow p$ as $t \rightarrow \infty$. Then, there exists a $C^{1}$ curve $d:[0, \tau] \rightarrow U$ such that $d(0)=c(0), d(\tau)=p$, and for every $t \in[0, \tau]$, the tangent vector $\dot{d}(t)$ coincides with $\operatorname{grad} f /\|\operatorname{grad} f\|$ at $d(t)$.

This lemma is a consequence of [1, Th. 6.1 (p. 242), Cor. 16.4 (p. 314)]. This completes the proof of Proposition 2.2.1.

\section{§. Tangential curves and their lifts}

Fix a Riemannian structure $g$ on $V$ and a vector field $X$ of class $C^{3}$ on $V$ such that $\omega(X)=1$. Let $\omega^{\prime}=-\mathscr{L}_{X} \omega$, and $\left\{\varphi_{s}\right\}$ the one-parameter group of transformations generated by $X$.

\subsection{Tangential curves and their lifts.}

Definition 3.1.1. A continuous curve $c$ in $V$ is called tangential if the image of $c$ is contained in a leaf. For a tangential curve $c:[0, \tau] \rightarrow V$ and $\eta \in \boldsymbol{R}$, suppose that there is a continuous function $\sigma:[0, \tau] \rightarrow \boldsymbol{R}$ such that $\eta=\sigma(0)$ and such that the curve $\delta:[0, \tau] \rightarrow V$ defined by $\delta(t)=\varphi_{\sigma(t)}(c(t))$, is tangential. Then $\mathfrak{D}$ is called the $\eta$-lift of $\mathfrak{c}$, and $\sigma$ is called the height parameter of the $\eta$-lift of $c$.

Let $\kappa$ be a positive number such that the inequality

$$
\kappa>\frac{1}{2}\left|\frac{\partial^{2}}{\partial s^{2}} \omega\left(\varphi_{s, *}(v)\right)\right|
$$

holds for all $v \in T_{1}(V)$ and all $s$ satisfying $|s| \leq 1$, where $T_{1}(V)$ denotes the tangent sphere bundle of $V$. This $\kappa$ has the following property. 
LEMMA 3.1.1. Let $\mathfrak{c}:[0, \tau] \rightarrow V$ be a tangential, piecewise $C^{1}$ curve such that $\|\dot{\mathfrak{c}}(t)\|=1, \omega^{\prime}(\dot{\mathfrak{c}}(t)) \leq 0$ for every $t \in[0, \tau]$ at which $\mathfrak{c}$ is differentiable. Let $\eta$ be a real number such that $0<|\eta|<1$. If the inequality

$$
\int_{0}^{\tau}\left(\exp \int_{c[0, u]} \omega^{\prime}\right) d u<\frac{1-|\eta|}{\kappa|\eta|}
$$

holds, then $\mathfrak{c}$ has the $\eta$-lift, whose height parameter $\sigma$ satisfies

$$
\sigma^{-}(t)<\sigma(t)<\sigma^{+}(t) \quad \text { for all } t \in(0, \tau],
$$

where

$$
\sigma^{ \pm}(t)=\left(\eta \exp \int_{c[0, t]} \omega^{\prime}\right) /\left(1 \mp \kappa \eta \int_{0}^{t}\left(\exp \int_{c[0, u]} \omega^{\prime}\right) d u\right)
$$

This lemma will be proved in 3.2.

Corollary 3.1.2. Let $\kappa$ be as above. Let $\mathrm{c}:[0, \tau] \rightarrow V$ be a tangential, piecewise $C^{1}$ curve such that $\|\dot{\mathfrak{c}}(t)\|=1, \omega^{\prime}(\dot{\mathfrak{c}}(t))=0$ for every $t \in[0, \tau]$ at which $\mathfrak{c}$ is differentiable. If a real number $\eta$ satisfies

$$
|\eta|<1 /(\kappa \tau+1)
$$

then $\mathrm{c}$ has the $\eta$-lift.

Proof. Since every tangential curve always has the 0 -lift, we may assume $\eta \neq 0$. The inequality $|\eta|<1 /(\kappa \tau+1)$ implies that $|\eta|<1$ and $\tau<(1-|\eta|) /(\kappa|\eta|)$. Therefore, since $\int_{c[0, u]} \omega^{\prime}=0$, the curve $c$ satisfies the assumption of Lemma 3.1.1. Hence $\mathfrak{c}$ has the $\eta$-lift.

3.2. Proof of Lemma 3.1.1. The proof will be preceded by four lemmas, 3.2.1.-3.2.4.

Lemma 3.2.1. Let $\eta \in \boldsymbol{R}$. Suppose that a tangential, $C^{1}$ curve c has the $\eta$-lift. Then the height parameter $s=\sigma(t)$ satisfies the differential equation

$$
\frac{d s}{d t}=-\omega\left(\varphi_{s, *}(\dot{c}(t))\right)
$$

with initial condition $s(0)=\eta$.

Proof. For the curve c: $[0, \tau] \rightarrow V$, consider the map $F:[0, \tau] \times \boldsymbol{R} \rightarrow V$ defined by $F(t, s)=\varphi_{s}(\mathfrak{c}(t)), t \in[0, \tau], s \in \boldsymbol{R}$. Let $\gamma$ be the vector field on 
$[0, \tau] \times \boldsymbol{R}$ defined by $\gamma(t, s)=\partial / \partial t-\omega\left(\varphi_{s, * \dot{c}}(t)\right) \partial / \partial s$ where $(t, s)$ is the canonical coordinates on $[0, \tau] \times \boldsymbol{R}$. It can be checked directly that $\left(F^{*} \omega\right) \gamma=0$. Let $\sigma:[0, \tau] \rightarrow \boldsymbol{R}$ be the height parameter of the $\eta$-lift of $c$. Let $d:[0, \tau]$ $\rightarrow[0, \tau] \times \boldsymbol{R}$ be the curve (of class $C^{1}$ ) defined by $d(t)=(t, \sigma(t))$. Since, by definition, the set $F \circ d([0, \tau])$ is contained in some leaf, we see that $\left(F^{*} \omega\right) \dot{d}(t)=0$ for all $t \in[0, \tau]$. Hence, we see that for each $t$, the two vectors $\gamma_{d(t)}, \dot{d}(t)$ are linearly dependent, because the one-form $F^{*} \omega$ is nonsingular. Using the identity $\dot{d}(t)=\partial / \partial t+(d \sigma / d t) \partial / \partial s$, we conclude that $\gamma_{d(t)}$ and $\dot{d}(t)$ coincide, and therefore the function $\sigma$ satisfies the differential equation $d \sigma / d t=-\omega\left(\varphi_{\sigma, *} \dot{c}(t)\right)$ with initial condition $\sigma(0)=\eta$.

LEMMA 3.2.2. Let $\kappa$ be the same positive number as in 3.1. Then the following inequalities hold:

$$
\omega^{\prime}(v) s-\kappa s^{2}<-\omega\left(\varphi_{s, *} v\right)<\omega^{\prime}(v) s+\kappa s^{2}
$$

for any nonzero $s$ with $|s| \leq 1$ and any $v \in T_{1}(V) \cap \omega^{-1}(0)$, i.e., any unit tangent vector $v$ such that $\omega(v)=0$.

Proof. Regarding $-\omega\left(\varphi_{s, *} v\right)$ as a function of $s$, by Taylor's formula, we have

$$
-\omega\left(\varphi_{s, *} v\right)=-\omega(v)-\left(\left(\frac{d}{d s}\right)_{s=0} \omega\left(\varphi_{s, *} v\right)\right) s-\frac{1}{2}\left(\left(\frac{d}{d s}\right)_{s=\xi}^{2} \omega\left(\varphi_{s, *} v\right)\right) s^{2},
$$

where $\xi \in[0, s]$. Since, by definition,

$$
\left(\frac{d}{d s}\right)_{s=0} \omega\left(\varphi_{s, *} v\right)=\left(\mathscr{L}_{X} \omega\right)(v)=-\omega^{\prime}(v)
$$

we have

$$
-\omega\left(\varphi_{s, *} v\right)=\omega^{\prime}(v) s-\frac{1}{2}\left(\left(\frac{d}{d s}\right)_{s=\xi}^{2} \omega\left(\varphi_{s, *} v\right)\right) s^{2}
$$

for $v \in \omega^{-1}(0)$. Hence we obtain the desired inequalities.

The following is a direct consequence of a classical result, due to Ricatti, on ordinary differential equations.

Lemma 3.2.3. Let $\kappa, \eta \in \boldsymbol{R} . \quad$ Let $\mathrm{c}:[0, \tau] \rightarrow V$ be a $C^{1}$ curve. Consider the differential equations

$$
\frac{d s}{d t}=\omega^{\prime}(\dot{\mathfrak{c}}(t)) s \pm \kappa s^{2}
$$


Then the solutions $s=\sigma^{ \pm}(t)$ with $\sigma^{ \pm}(0)=\eta$ are given by

$$
\sigma^{ \pm}(t)=\left(\eta \exp \int_{c[0, t]} \omega^{\prime}\right) /\left(1 \mp \kappa \eta \int_{0}^{t}\left(\exp \int_{c[0, u]} \omega^{\prime}\right) d u\right) \text {. }
$$

LEMMA 3.2.4. Let $\kappa$ be the same positive number as in 3.1. Let $\eta$ be a real number such $0<|\eta|<1$. Let $c:[0, \tau] \rightarrow V$ be a tangential, $C^{1}$ curve such that $\|\dot{\mathfrak{c}}(t)\|=1$, $\boldsymbol{\prime}^{\prime}(\dot{\mathfrak{c}}(t)) \leq 0$ for every $t \in[0, \tau]$, and such that the inequality

$$
\int_{0}^{\tau}\left(\exp \int_{c[0, u]} \omega^{\prime}\right) d u<\frac{1-|\eta|}{\kappa|\eta|}
$$

is satisfied. Then c has the $\eta$-lift, whose height parameter $\sigma=\sigma(t)$ satisfies the inequalities

$$
\sigma^{-}(t)<\sigma(t)<\sigma^{+}(t) \quad \text { for all } t \in(0, \tau],
$$

where

$$
\sigma^{ \pm}(t)=\left(\eta \exp \int_{c[0, t]} \omega^{\prime}\right) /\left(1 \mp \kappa \eta \int_{0}^{t}\left(\exp \int_{c[0, u]} \omega^{\prime}\right) d u\right)
$$

Proof. For simplicity we assume $\eta>0$, the proof in the case $\eta<0$ being similar. By Lemma 3.2.3, the solutions of the differential equations $d s / d t=\omega^{\prime}(\dot{\mathfrak{c}}(t)) s \pm \kappa s^{2}$ with initial condition $s(0)=\eta$, are given by $s=\sigma^{ \pm}(t)$. It is clear that $0<\sigma^{-}(t)$ for $t \in[0, \tau]$. Since, by assumption, $\int_{0}^{t}\left(\exp \int_{c[0, u]} \omega^{\prime}\right) d u<(1-\eta) /(\kappa \eta)$ for $t \in[0, \tau]$, we see that $\sigma^{+}(t)<\exp \int_{c[0, t]} \omega^{\prime}$ for $t \in[0, \tau]$, and therefore $\sigma^{+}(t)<1$ for $t \in[0, \tau]$. Note that by Lemma 3.2.2, the following inequalities hold:

$$
\omega^{\prime}(\dot{\mathfrak{c}}(t)) s-\kappa s^{2}<-\omega\left(\varphi_{s, *} \dot{\mathfrak{c}}(t)\right)<\omega^{\prime}(\dot{\mathfrak{c}}(t)) s+\kappa s^{2}
$$

for $s \in(0,1]$ and $t \in[0, \tau]$. Using these inequalities and Lemma 3.2.1, and comparing the functions $\sigma^{ \pm}(t)$ and the solution $s=\sigma(t)$ of the differential equation $d s / d t=-\omega\left(\varphi_{s, *} \dot{c}(t)\right)$ with initial condition $s(0)=\eta$, we can conclude that the curve $c$ has the $\eta$-lift, whose height parameter $\sigma$ satisfies the inequalities $\sigma^{-}(t)<\sigma(t)<\sigma^{+}(t)$ for $t \in(0, \tau]$, as desired.

Proof of Lemma 3.1.1. Using Lemma 3.2.4, we can easily prove our lemma.

3.3. Admissible tangential curves. For the Riemannian, foliated manifold $(V, \omega, \mathfrak{g})$, it is clear that there is a vector field $Y$ (of class $C^{2}$ ) on $V$ 
satisfying the following conditions:

(i ) $\omega(Y)=0$ (i.e., $\omega_{x}\left(Y_{x}\right)=0$ for all $x \in V$ );

(ii) $\omega^{\prime}(v)=\mathfrak{g}(Y, v)$ for all $v \in \omega^{-1}(0)$, i.e., $\omega_{x}^{\prime}(v)=\mathfrak{g}\left(Y_{x}, v\right)$ for every $x \in V$ and every tangent vector $v$ at $x$ such that $\omega_{x}(v)=0$.

We observe that for each leaf $L$ of $(V, \omega)$, the vector field $Y$ induces a vector field $Y \mid L$ on $L$, which is the dual vector field of $\omega^{\prime}$ with respect to the Riemannian structure $g \mid L$, and hence that, by Lemma 2.1.1, the vector field $Y$ is determined independently of the choice of $X$.

DEFINITION 3.3.1. This vector field $Y$ is called the leaf-gradient field of $(V, \omega, \mathrm{g})$.

By Lemma 2.1.1, we note that the set of singular points of $Y$ coincides with the critical cycle $\Sigma$ of $(V, \omega)$.

Definition 3.3.2. Let $Y$ be as above. A tangential curve $a$ will be called an admissible tangential curve of $(V, \omega, \mathfrak{g})$ if $\mathfrak{a}$ is piecewise $C^{1}$ and if, for any $x=\mathfrak{a}(t)$ at which $Y$ does not vanish, the tangent vector $\dot{a}(t)$ (exists and) coincides with $-Y_{x} /\left\|Y_{x}\right\|$.

Proposition 3.3.1. Let $Y$ be the leaf-gradient field of $(V, \omega, \mathfrak{g})$. Let $Z$ be the vector field on $V-\Sigma$ defined by

$$
Z_{x}=-Y_{x} /\left\|Y_{x}\right\|^{2}
$$

for $x \in V-\Sigma$. Let $W$ be an open subset in $V$ such that $\mathrm{Cl} W \cap \Sigma=\phi$. Then for any positive number $\varepsilon$, there exists a positive number $h$ having the following property: for any $\eta \in(-h, h)$ and any integral curve $z:[0, \tau] \rightarrow W$ of $Z$, there is the $\eta$-lift of $z$, whose height parameter $\sigma$ satisfies

$$
|\eta| e^{-(1+\varepsilon) t} \leq|\sigma(t)| \leq|\eta| e^{-(1-s) t} \quad \text { for all } t \in[0, \tau] .
$$

Proof. Let $\varepsilon>0$. Applying the mean value theorem to the function

$$
s \mapsto-\omega\left(\varphi_{s, *} Z_{x}\right)-\omega^{\prime}\left(Z_{x}\right) s,
$$

where $x \in \mathrm{Cl} W$, and using the identity

$$
\left(\frac{d}{d s}\right)_{0} \omega\left(\varphi_{s, *} Z_{x}\right)=-\omega^{\prime}\left(Z_{x}\right)
$$

and the compactness of $\mathrm{Cl} W$, we can find a positive number $h$ such that 


$$
\left|-\omega\left(\varphi_{s, *} Z_{x}\right) s^{-1}-\omega^{\prime}\left(Z_{x}\right)\right|<\varepsilon
$$

for any $x \in \mathrm{Cl} W$ and any nonzero $s \in(-h, h)$. Hence, for this number $h$, we have

$$
-\varepsilon-1<-\omega\left(\varphi_{s, *} Z_{x}\right) s^{-1}<\varepsilon-1
$$

for $x \in \mathrm{Cl} W$ and nonzero $s \in(-h, h)$, because $\omega^{\prime}\left(Z_{x}\right)=-1$. Let $z:[0, \tau]$ $\rightarrow W$ be an integral curve of $Z$. It is clear that

$$
-\varepsilon-1<-\omega\left(\varphi_{s, *} \dot{z}(t)\right) s^{-1}<\varepsilon-1
$$

for nonzero $s \in(-h, h)$, and therefore

$$
\begin{array}{ll}
-(1+\varepsilon) s<-\omega\left(\varphi_{s, *} \dot{z}(t)\right)<-(1-\varepsilon) s & \text { for } s \in(0, h), \\
-(1+\varepsilon) s>-\omega\left(\varphi_{s, *} \dot{z}(t)\right)>-(1-\varepsilon) s & \text { for } s \in(-h, 0) .
\end{array}
$$

Let $\eta \in(-h, h)$. Similarly to the proof of Lemma 3.2.4, using Lemma 3.2.1 and comparing the functions $\eta e^{-(1 \pm \varepsilon) t}$ and the solution of the differential equation $d s / d t=-\omega\left(\varphi_{s, *} \dot{z}(t)\right)$ with initial condition $s(0)=\eta$, we can conclude that the curve $z$ has the $\eta$-lift, whose height parameter $\sigma$ satisfies $|\eta| e^{-(1+\varepsilon) t} \leq|\sigma(t)| \leq|\eta| e^{-(1-\varepsilon) t}$ for $t \in[0, \tau]$, as desired.

\section{§4. $\omega$-preferred Riemannian structures}

4.1. $\omega$-preferred Riemannian structures. We shall say that $\left(U, f ; x^{1}\right.$, $\left.\cdots, x^{n+1}\right)$ is an $\mathscr{F}$-chart $(a t p)$ of class $C^{s}(1 \leq s \leq r+1)$ if $\left(x^{1}, \cdots, x^{n+1}\right)$ is a local coordinate system of class $C^{s}$ in a neighborhood $U$ of $p \in V$, and if the identity $\omega \mid U=f d x^{n+1}$ holds for some positive-valued $C^{s-1}$ function $f$ on $U$.

DeFinition 4.1.1. Let $\lambda$ be an integer on the range $0,1, \cdots, n$. An $\mathscr{F}$-chart $\left(U, f ; x^{1}, \cdots, x^{n+1}\right)$ of class $C^{4}$ is said to be of type ( $\lambda$ ) if $U$ is mapped onto a neighborhood of the origin in $\boldsymbol{R}^{n+1}$, by $\left(x^{1}, \cdots, x^{n+1}\right)$ and if the identity

$$
\log f=g\left(x^{n+1}\right)-\left(x^{1}\right)^{2}-\cdots-\left(x^{\lambda}\right)^{2}+\left(x^{\lambda+1}\right)^{2}+\cdots+\left(x^{n}\right)^{2}
$$

holds throughout $U$, where $g$ is some suitable function defined on an open set in $\boldsymbol{R}$.

Let $\mu$ be an integer on the range $0,1, \cdots, n-1$. An $\mathscr{F}$-chart $(U$, $\left.f ; x^{1}, \cdots, x^{n+1}\right)$ of class $C^{4}$ is said to be of type $(*, \mu)$ if $U$ is mapped onto a neighborhood of the origin in $\boldsymbol{R}^{n+1}$, by $\left(x^{1}, \cdots, x^{n+1}\right)$ and if the 
identity

$$
\log f=g\left(x^{n}, n^{n+1}\right)-\left(x^{1}\right)^{2}-\cdots-\left(x^{\mu}\right)^{2}+\left(x^{\mu+1}\right)^{2}+\cdots+\left(x^{n-1}\right)^{2}
$$

holds throughout $U$, where $g$ is some $C^{3}$ function which is defined on an open set $U_{0}$ is $\boldsymbol{R}^{2}$ and satisfies the following conditions:

(i) the set $\Sigma(g)$ defined by

$$
\Sigma(g)=\left\{\left(x^{n}, x^{n+1}\right) \in U_{0} \mid \partial g\left(x^{n}, x^{n+1}\right) / \partial x^{n}=0\right\},
$$

coincides with either the set

$$
\left\{\left(x^{n}, x^{n+1}\right) \in U_{0} \mid\left(x^{n}\right)^{2}-x^{n+1}=0\right\}
$$

or the set

$$
\left\{\left(x^{n}, x^{n+1}\right) \in U_{0} \mid\left(x^{n}\right)^{2}+x^{n+1}=0\right\} ;
$$

(ii)

$$
\begin{aligned}
\partial^{2} g\left(x^{n}, x^{n+1}\right) /\left(\partial x^{n}\right)^{2}=0 & \text { at }\left(x^{n}, x^{n+1}\right)=(0,0), \\
\partial^{3} g\left(x^{n}, x^{n+1}\right) /\left(\partial x^{n}\right)^{3} \neq 0 & \text { at }\left(x^{n}, x^{n+1}\right)=(0,0), \\
\partial^{2} g\left(x^{n}, x^{n+1}\right) / \partial x^{n} \partial x^{n+1} \neq 0 & \text { at }\left(x^{n}, x^{n+1}\right)=(0,0), \\
\partial^{2} g\left(x^{n}, x^{n+1}\right) /\left(\partial x^{n}\right)^{2} \neq 0 & \text { at any }\left(x^{n}, x^{n+1}\right) \in \Sigma(g)-\{(0,0)\} .
\end{aligned}
$$

Definition 4.1.2. A Riemannian structure $g$ of class $C^{2}$ on $V$ will be called an $\omega$-preferred Riemannian structure if for any $p \in \Sigma$, there exists an $\mathscr{F}$-chart-at-p $\left(U, f ; x^{1}, \cdots, x^{n+1}\right)$ of class $C^{4}$ satisfying one of the following conditions:

(i) $\left(U, f ; x^{1}, \cdots, x^{n+1}\right)$ is of type $(\lambda)$ for some $\lambda$ and satisfies

$$
\mathfrak{g}\left(\frac{\partial}{\partial x^{i}}, \frac{\partial}{\partial x^{j}}\right)=\delta_{i j} \quad \text { for } 1 \leq i, j \leq n ;
$$

(ii) $\left(U, f ; x^{1}, \cdots, x^{n+1}\right)$ is of type $(*, \mu)$ for some $\mu$ and satisfies

$$
\begin{array}{ll}
\mathfrak{g}\left(\frac{\partial}{\partial x^{i}}, \frac{\partial}{\partial x^{j}}\right)=\delta_{i j} & \text { for } 1 \leq i, j \leq n-1, \\
\mathfrak{g}\left(\frac{\partial}{\partial x^{i}}, \frac{\partial}{\partial x^{n}}\right)=0 & \text { for } 1 \leq i \leq n-1 .
\end{array}
$$

( $\delta_{i j}$ is the Kronecker delta.)

Proposition 4.1.1. If $\omega$ satisfies Condition (T), then $V$ admits an $\omega$-preferred Riemannian structure.

We shall prove this proposition in $\S 8$. 
The following three lemmas are important for the proofs of the main theorems. Let $X, \omega^{\prime}$ be as in $\S 3$.

LEMMA 4.1.2. Suppose that an w-preferred Riemannian structure $\mathrm{g}$ is given on $V$. Then there exist two positive constants $\alpha_{*}, \tau_{*}$ such that for any admissible tangential curve $\mathfrak{a}:[0, \tau] \rightarrow V$ of $(V, \omega, \mathfrak{g})$, the following inequality holds:

$$
\int_{a[0, t]} \omega^{\prime}<-\alpha_{*} t \quad \text { for any } t \text { satisfying } \tau_{*} \leq t \leq \tau .
$$

This lemma will be proved in 4.3. The following lemma concerning the lifts of admissible tangential curves is fundamental.

LEMMA 4.1.3. Suppose that an w-preferred Riemannian structure $\mathfrak{g}$. is given on $V$. Then there exist two constants $\eta_{*}, \beta_{*}$ such that $0<\eta_{*}<1$, $0<\beta_{*}<1 / \eta_{*}$ and such that, if $\eta$ is a real number satisfying $0<|\eta|<\eta_{*}$, then any admissible tangential curve $\mathfrak{a}:[0, \tau] \rightarrow V$ of $(V, \omega, \mathfrak{g})$ has the $\eta$ lift, whose height parameter $\sigma$ satisfies the following inequalities:

$$
\sigma_{*}^{-}(t)<\sigma(t)<\sigma_{*}^{+}(t) \quad \text { for all } t \in[0, \tau],
$$

where $\sigma_{*}^{ \pm}(t)$ are defined by

$$
\sigma_{*}^{ \pm}(t)=\left(\eta \exp \int_{a[0, t]} \omega^{\prime}\right) /\left(1 \mp \eta \beta_{*}\right)
$$

Proof. Let $\alpha_{*}, \tau_{*}$ be the same constants as Lemma 4.1.2, and let $\kappa$ be the same positive number as in 3.1. Put

$$
a=\tau_{*}+\alpha_{*}^{-1}, \quad \beta_{*}=\kappa a, \quad \eta_{*}=1 /(1+\kappa a) .
$$

Now, let $\eta$ be a real number satisfying $0<|\eta|<\eta_{*}$, and let $a:[0, \tau] \rightarrow$ $V$ be an admissible tangential curve. It is clear that $a<(1-|\eta|) /(\kappa|\eta|)$, and that

$$
\int_{0}^{t}\left(\exp \int_{a[0, u]} \omega^{\prime}\right) d u \leq t, \quad \text { because } \int_{a[0, u]} \omega^{\prime} \leq 0
$$

First, we shall prove that

$$
\int_{0}^{t}\left(\exp \int_{a[0, u]} \omega^{\prime}\right) d u<a \quad \text { for all } t \in[0, \tau] .
$$

In the case $\tau<\tau_{*}$, clearly this inequality holds, because $\tau_{*}<a$. Therefore we assume $\tau \geq \tau_{*}$. From Lemma 4.1 .2 we know that 


$$
\exp \int_{\mathfrak{a}[0, t]} \omega^{\prime}<\exp \left(-\alpha_{*} t\right) \quad \text { for } t \geq \tau_{*} .
$$

Hence, for $t \in[0, \tau]$ we have

$$
\begin{aligned}
\int_{0}^{t}\left(\exp \int_{a[0, u]} \omega^{\prime}\right) d u & =\int_{0}^{\tau_{*}}+\int_{\tau_{*}}^{t}<\tau_{*}+\int_{0}^{\infty}\left(\exp \left(-\alpha_{*} u\right)\right) d u \\
& =\tau_{*}+\alpha_{*}^{-1}=a, \quad \text { as desired } .
\end{aligned}
$$

Hence, from Lemma 3.1.1 we see that the curve a has the $\eta$-lift, whose height parameter $\sigma$ satisfies the inequalities $\sigma^{-}(t)<\sigma(t)<\sigma^{+}(t)$ for $t \in(0, \tau]$, where $\sigma^{ \pm}$are the same functions as in Lemma 3.1.1. It is easy to verify that

$$
\sigma_{*}^{-}(t)<\sigma^{-}(t), \sigma^{+}(t)<\sigma_{*}^{+}(t) \quad \text { for all } t \in[0, \tau] \text {, }
$$

because

$$
\kappa \int_{0}^{t}\left(\exp \int_{\mathrm{a}[0, u]} \omega^{\prime}\right) d u<\beta_{*}
$$

Hence we conclude that $\sigma_{*}^{-}(t)<\sigma(t)<\sigma_{*}^{+}(t)$ for all $t \in[0, \tau]$. Our lemma is proved.

Remark 4.1.1. In the preceding lemma, putting $\beta_{*}^{ \pm}=1 /\left(1 \mp \eta_{*} \beta_{*}\right)$, we observe that $0<\beta_{*}^{-}<1<\beta_{*}^{+}$, and that the inequalities

$$
\beta_{*}^{-} \cdot|\eta| \cdot \exp \int_{a[0, t]} \omega^{\prime}<|\sigma(t)|<\beta_{*}^{+} \cdot|\eta| \cdot \exp \int_{a[0, t]} \omega^{\prime}
$$

hold.

The next lemma gives us the existence of the maximal admissible tangential curves.

LEMMA 4.1.4. Suppose that an w-preferred Riemannian structure $\mathfrak{g}$ is given on $V$. Then for any point $p$ of $V$, there exists an admissible tangential curve a which passes through $p$ and satisfies one of the following:

(i) a is defined on $(-\infty, \infty)$;

(ii) $\mathfrak{a}$ is defined on $(-\infty, 0]$, and $\mathfrak{a}(0) \in \Sigma_{0}$;

(iii) $\mathfrak{a}$ is defined on $[0, \infty)$, and $\mathfrak{a}(0) \in \Sigma_{n}$;

(iv) $a$ is defined on a finite interval $[0, \tau]$, and $\mathfrak{a}(0) \in \Sigma_{n}, \mathfrak{a}(\tau) \in \Sigma_{0}$.

This will be proved in 4.3 . 
4.2. $\quad \boldsymbol{Y}$-preferred neighborhoods of $\boldsymbol{\Sigma}$. Let $\mathrm{g}$ be a Riemannian structure on $V$, and let $Y$ be the leaf-gradient field of $(V, \omega, g)(3.3)$.

DEFINITION 4.2.1. An open set $T^{*}$ in $V$ will be called a $Y$-preferred neighborhood of the critical cycle $\Sigma$ if the following nine conditions are satisfied:

(i) $\Sigma \subset T^{*}$.

(ii) There exist two positive constants $a_{1}^{*}, a_{2}^{*}$ such that, if an admissible tangential curve $a:[0, \tau] \rightarrow V$ satisfies $\operatorname{Im} a \subset T^{*}$, then $\tau<a_{1}^{*}$, and such that, if an admissible tangential curve $a:[-\tau, \tau] \rightarrow V$ satisfies the following conditions:

$$
\mathfrak{a}(0) \in \mathrm{Cl} T^{*} \cap\left(V-T^{*}\right) ; \quad \tau<a_{2}^{*},
$$

then

$$
\text { either } \quad \mathfrak{a}([-\tau, 0]) \cap T^{*}=\emptyset \quad \text { or } \quad \mathfrak{a}([0, \tau]) \cap T^{*}=\emptyset .
$$

(iii) For any $x \in T^{*}$, there exists an admissible tangential curve $\mathfrak{a}:[0, \tau] \rightarrow V$ such that $x \in \operatorname{Im} \mathfrak{a} \subset \mathrm{Cl} T^{*}$, and such that one of the following is satisfied:

$$
\begin{array}{lll}
\mathfrak{a}(0) \notin T^{*} & \text { and } & \mathfrak{a}(\tau) \notin T^{*} ; \\
\mathfrak{a}(0) \in \Sigma_{n} & \text { and } & \mathfrak{a}(\tau) \notin T^{*} ; \\
\mathfrak{a}(0) \notin T^{*} & \text { and } & \mathfrak{a}(\tau) \in \Sigma_{0} ; \\
\mathfrak{a}(0) \in \Sigma_{n} & \text { and } & \mathfrak{a}(\tau) \in \Sigma_{0} .
\end{array}
$$

(iv) There exists a positive constant $a_{3}^{*}$ such that for any

$$
x \in \mathrm{Cl} T^{*} \cap\left(V-T^{*}\right),
$$

there is an admissible tangential curve $a:[0, \tau] \rightarrow V$ satisfying the following conditions:

$$
\tau>a_{3}^{*} ; \quad \operatorname{Im} \mathfrak{a} \cap T^{*}=\emptyset ;
$$

and

$$
\text { either } a(0)=x \text { or } \quad \mathfrak{a}(\tau)=x \text {. }
$$

Here in order to describe (v)-(viii), we introduce the following word: A nonempty subset $J^{*}$ of $V$ will be called a $T^{*}$-vein if there is a vein $J$ such that $J^{*}$ coincides with some connected component of the subset $T^{*} \cap J$ in $J$. For a $T^{*}$-vein $J^{*}$, we denote by $\operatorname{diam}_{J^{*}}\left(J^{*}\right)$ the supremum 
of the distances in $J^{*}$ between any pairs of points of $J^{*}$.

(v) There exist three positive constants $b_{1}^{*}, b_{2}^{*}, b_{3}^{*}$ such that

$$
\operatorname{diam}_{J^{*}}\left(J^{*}\right)<b_{1}^{*}, \quad \operatorname{mes}_{n-1}\left(J^{*}\right)<b_{3}^{*}
$$

for any $T^{*}$-vein $J^{*}$, and such that

$$
\operatorname{mes}_{n-1}\left(J^{*}\right)>b_{2}^{*}
$$

for any noncompact $T^{*}$-vein $J^{*}$, where $\operatorname{mes}_{n-1}\left(J^{*}\right)$ is the total measure of the $(n-1)$-manifold $J^{*}-\left(J^{*} \cap \Sigma\right)$ with the Riemannian structure induced by $\mathrm{g}$.

(vi) If a $T^{*}$-vein $J^{*}$ is compact, then there exists an imbedding $i: D^{n} \rightarrow L$ of a closed unit $n$-disk to a leaf such that for each $\varepsilon$-sphere $S^{n-1}(\varepsilon), 0 \leq \varepsilon \leq 1$, the image $i\left(S^{n-1}(\varepsilon)\right)$ is a vein and $i\left(S^{n-1}\left(\varepsilon^{\prime}\right)\right)=J^{*}$ for some $\varepsilon^{\prime}$.

(vii) (local $Y$-invariance) Let $a:[0, \tau] \rightarrow V$ be an admissible tangential curve such that $\operatorname{Im} a \subset T^{*}$, and let $\mathfrak{a}^{\prime}:\left[0, \tau^{\prime}\right] \rightarrow V$ be another admissible tangential curve such that

$$
\text { either } \mathfrak{a}^{\prime}(0) \in J^{*}(\mathfrak{a}(0)) \text { or } \quad \mathfrak{a}^{\prime}\left(\tau^{\prime}\right) \in J^{*}(\mathfrak{a}(\tau)) \text {, }
$$

where $J^{*}(\mathfrak{a}(0)), J^{*}(\mathfrak{a}(\tau))$ are the $T^{*}$-veins containing $\mathfrak{a}(0), \mathfrak{a}(\tau)$, respectively. If

$$
\int_{a^{\prime}\left[0, \tau^{\prime}\right]} \omega^{\prime}=\int_{a[0, \tau]} \omega^{\prime},
$$

then $\operatorname{Im} \mathfrak{a}^{\prime} \subset T^{*}$.

(viii) There exists a positive constant $d_{1}^{*}$, and for each $x \in T^{*}$, there exists an imbedded closed $n$-disk $Q$ in the leaf $L(x)$ through $x$, such that $Q$ contains the connected component of $T^{*} \cap L(x)$ containing $x$, and such that for a function $h: Q \rightarrow \boldsymbol{R}$ satisfying

$$
\omega^{\prime} \mid Q=d h,
$$

the following inequality holds:

$$
d_{1}^{*} \cdot\left(h_{0}-h_{\tau}\right) \inf _{t \in[0, \tau]} \operatorname{mes}_{n-1}\left(\left(h^{-1}\left(h_{t}\right) \cap T^{*}\right)\right) \leq \operatorname{mes}_{n}\left(\left(h^{-1}\left(\left[h_{\tau}, h_{0}\right]\right) \cap T^{*}\right)\right)
$$

for any admissible tangential curve $\mathfrak{a}:[0, \tau] \rightarrow V$ such that $\operatorname{Im} \mathfrak{a} \subset T^{*}$, where $h_{t}=h(\mathfrak{a}(t))$, and $(())$ denote the connected components of the sets in the parentheses, containing the point $a(t)$, and where the right side of the inequality denotes the total measure of the $n$-manifold $\left(\left(h^{-1}\left(\left(h_{\tau}, h_{0}\right)\right) \cap T^{*}\right)\right)$ 
with the Riemannian structure induced by $\mathrm{g}$.

(ix) For any vein $J$, the subset $J-\left(J \cap T^{*}\right)$ is an $(n-1)$-submanifold with or without boundary, of $J-(J \cap \Sigma)$.

Proposition 4.2.1. Suppose that an $\omega$-preferred Riemannian structure $\mathfrak{g}$ is given on $V$. Let $Y$ be the leaf-gradient field of $(V, \omega, \mathfrak{g})$. Then for any neighborhood $W$ of $\Sigma$, there exists a $Y$-preferred neighborhood $T^{*}$ of $\Sigma$ such that $T^{*} \subset W$.

We shall prove this proposition in $\S 8$.

4.3. Proofs of Lemmas 4.1.2 and 4.1.4. For the $\omega$-preferred Riemannian structure $g$ and the leaf-gradient field $Y$ of $(V, \omega, \mathfrak{g})$, let $T^{*}$ be a $Y$ preferred neighbourhood of $\Sigma$.

Proof of Lemma 4.1.2. Put

$$
\eta=\min _{x \in V-T^{*}}\left\|Y_{x}\right\|, \quad a=a_{1}^{*}+a_{2}^{*}, \quad \tau_{*}=2 a, \quad \alpha_{*}=\frac{\eta a_{2}^{*}}{2 a},
$$

where $a_{1}^{*}, a_{2}^{*}$ are the same constants as in (ii) of Definition 4.2.1. Now let $\mathfrak{a}:[0, \tau] \rightarrow V$ be an admissible tangential curve. Let $\tau_{*} \leq t \leq \tau$. Choosing an integer $k$ so that $0 \leq t-k a<a$, we have

$$
\int_{a[0, t]} \omega^{\prime}=\int_{0}^{t} \omega^{\prime}(\dot{\mathrm{a}}(u)) d u=\int_{0}^{a}+\int_{a}^{2 a}+\cdots+\int_{k a}^{t} .
$$

For $u \in[0, \tau]$ such that $a(u) \in V-T^{*}$, we observe that

$$
\begin{aligned}
\omega^{\prime}(\dot{\mathfrak{a}}(u)) & =\omega^{\prime}\left(-Y_{\mathrm{a}(u)} /\left\|Y_{\mathrm{a}(u)}\right\|\right)=\mathfrak{g}(Y,-Y /\|Y\|)_{\mathrm{a}(u)} \\
& =-\left\|Y_{\mathrm{a}(u)}\right\| \leq-\eta .
\end{aligned}
$$

We also see that $\omega^{\prime}(\dot{\mathfrak{a}}(u)) \leq 0$ for all $u \in[0, \tau]$. Hence

$$
\int_{k a}^{t} \omega^{\prime}(\dot{\mathfrak{a}}(u)) d u \leq 0 \text {. }
$$

By (ii) of Definition 4.2.1, we have for each $i=1, \cdots, k$,

$$
\int_{i a}^{(i+1) a} \omega^{\prime}(\dot{\mathfrak{a}}(u)) d u \leq-\eta a_{2}^{*} .
$$

Since $k>(t-a) / a$, using the fact that $(t-a) / t \geq 1 / 2$, we have

$$
\int_{a[0, t]} \omega^{\prime} \leq-k \eta a_{2}^{*}<-\frac{t-a}{a t} \cdot \eta a_{2}^{*} t \leq-\frac{\eta a_{2}^{*}}{2 a} \cdot t=-\alpha_{*} t,
$$


which proves our lemma.

Proof of Lemma 4.1.4. Since every admissible tangential curve in $V-T^{*}$ is an integral curve of the vector field $-Y /\|Y\|$ defined on $V-T^{*}$, the lemma is a direct consequence of the conditions (iii) and (iv) in Definition 4.2.1.

\section{§5. Proof of Theorem I}

Throughout $\S 5, \S 6$, and $\S 7$, we assume that Condition (T) is satisfied. Let $g$ be an $\omega$-preferred Riemannian structure. (Such a Riemannian structure exists by Proposition 4.1.1.) Let $X$ be a vector field of class $C^{r}$ on $V$ such that $\omega(X)=1$, and $\left\{\varphi_{s}\right\}$ the one-parameter group of transformations generated by $X$. Let $Y$ be the leaf-gradient field of $(V, \omega, \mathfrak{g})$ (see 3.3) and put $\omega^{\prime}=-\mathscr{L}_{X} \omega$.

5.1. Proof of Theorem I. Let $\eta_{*}, \beta_{*}$ be the same constants as in Lemma 4.1.3. We begin with a lemma.

LEMMA 5.1.1. There exists a positive constant $\zeta_{*}$ having the following property: for any $x, y \in V$ satisfying dis $(x, y)<\zeta_{*}$, there is a real number $\eta$ with $|\eta|<\eta_{*}$ such that the point $\varphi_{\eta}(x)$ is contained in the leaf through $y$.

This is an easy consequence of local triviality of the foliated structure.

Proof of Theorem I. Note that $\Sigma_{0}$ is a one-manifold and is transversal to leaves. From Remark 2.2.1 we know that there exists a leaf $L$ containing a point $p \in \Sigma_{0}$, such that the closed one-form $\omega^{\prime} \mid L$ on $L$ is proper. For $i=0,1, \cdots, n$, and $q \in L$, note that $q$ belongs to $\Sigma_{i}$ if and only if $q$ is a nondegenerate singular point of $\omega^{\prime} \mid L$, with index $i$, because for an $\mathscr{F}$-chart $\left(U, f ; x^{1}, \cdots, x^{n+1}\right)$ and $q \in U \cap \Sigma$, the following identities hold:

$$
\frac{\partial^{2} \log f}{\partial x^{i} \partial x^{j}}(q)=\frac{1}{f} \frac{\partial^{2} f}{\partial x^{i} \partial x^{j}}(q) \quad \text { for } i, j=1, \cdots, n .
$$

Hence we can apply Proposition 2.2.1 to $\left(L, \omega^{\prime} \mid L\right)$.

We shall prove $L$ is compact. Since $\Sigma_{0}$ is transversal to leaves, there is a positive number $\delta$ such that for any nonzero $s$ with $|s|<\delta$, the leaf through the point $\varphi_{s}(p)$ intersects $\Sigma_{0}-\{p\}$. Suppose that $L$ were not compact. Then there would exist $x \in L$ and $\eta \in R$ having the follow- 
ing properties:

$$
0<\eta<\eta_{*}, \quad \eta /\left(1-\eta \beta_{*}\right)<\delta, \quad \varphi_{\eta}(x) \in L .
$$

By (v) of Proposition 2.2.1, there would exist an admissible tangential curve $a:[0, \tau] \rightarrow V$ such that $x=\mathfrak{a}(0), p=\mathfrak{a}(\tau)$. By Lemma 4.1.3, there would exist the $\eta$-lift of $a$, with the height parameter $\sigma$ satisfying

$$
0<\sigma(\tau)<\eta /\left(1-\eta \beta_{*}\right) .
$$

Since the leaf through $\varphi_{\sigma(\tau)}(p)$ would be nothing other than $L$, the leaf $L$ would intersect $\Sigma_{0}-\{p\}$, which contradicts (iv) of Proposition 2.2.1. Thus $L$ is compact. Since, by (ii) of Proposition 2.2.1, $L$ is simply connected, our theorem follows from the Reeb global stability theorem [1, (B, III, 11)].

5.2. The orientation of $\boldsymbol{\Sigma}$. We orient $\Sigma$ as follows. Let $p \in \Sigma$, and let $\left(U, f ; x^{1}, \cdots, x^{n+1}\right)$ be an $\mathscr{F}$-chart at $p$. Denote by $\mathbf{0}$ the origin of $\boldsymbol{R}^{n}$. Consider the map $\partial f: U \rightarrow \boldsymbol{R}^{n}$ defined by

$$
\partial f(x)=\left(\frac{\partial f}{\partial x^{1}}(x), \cdots, \frac{\partial f}{\partial x^{n}}(x)\right) .
$$

Note that $U \cap \Sigma=(\partial f)^{-1}(\mathbf{0})$. Let

$$
(\partial f)_{* p}: T_{p}(U) \rightarrow T_{0}\left(\boldsymbol{R}^{n}\right)=\boldsymbol{R}^{n}
$$

be the map induced by $\partial f$, where $T_{p}(U), T_{0}\left(\boldsymbol{R}^{n}\right)$ are the tangent spaces of $U, \boldsymbol{R}^{n}$ at $p, \mathbf{0}$, respectively. It follows from Condition (T) that the $\operatorname{map}(\partial f)_{* p}$ is onto. Since the kernel of $(\partial f)_{* p}$ coincides with the tangent space $T_{p}(\Sigma)$ of $\Sigma$ at $p$, we have a direct sum

$$
T_{p}(U)=T_{p}(\Sigma)^{\perp} \oplus T_{p}(\Sigma),
$$

and we see that the restriction

$$
(\partial f)_{* p} \mid T_{p}(\Sigma)^{\perp}: T_{p}(\Sigma)^{\perp} \rightarrow \boldsymbol{R}^{n}
$$

is an isomorphism. This isomorphism and the standard orientation of $\boldsymbol{R}^{n}$ define an orientation $\xi_{1}(p)$ of $T_{p}(\Sigma)^{\perp}$. Let $\xi(p)$ be an orientation of $T_{p}(U)$ determined by the base

$$
\left(\frac{\partial}{\partial x^{1}}, \cdots, \frac{\partial}{\partial x^{n+1}}\right)_{p}
$$


Then we define the orientation $\xi_{2}(p)$ of $T_{p}(\Sigma)$ so that

$$
\xi(p)=\xi_{1}(p) \oplus \xi_{2}(p),
$$

i.e., so that the orientation $\xi(p)$ agrees with the orientation determined by a base $\left(v_{1}, \cdots, v_{n}, v_{n+1}\right)$ of $T_{p}(U)$, where $\left(v_{1}, \cdots, v_{n}\right)$ is a representative of $\xi_{1}(p)$ and $v_{n+1}$ is a representative of $\xi_{2}(p)$. (Orientation is to be understood as an equivalence class of basis.) It can be checked directly that the orientation $\xi_{2}(p)$ of $T_{p}(\Sigma)$ is defined independently of the choice of $\mathscr{F}$ charts and varies continuously with $p \in \Sigma$. Hence $\xi_{2}$ defines an orientation of $\Sigma$. In 5.3, we shall suppose that $\Sigma$ is oriented by this $\xi_{2}$.

5.3. Corollaries. Let $S^{1}$ be an oriented, one-dimensional sphere of class $C^{r}$, and let $\theta$ be a nonsingular one-form of class $C^{r-1}$ on $S^{1}$ such that the integral of $\theta$ on $S^{1}$ is equal to 1 .

As another version of Theorem I, we have:

THEOREM I'. Let the hypotheses be as in Theorem I. Then there exists a $C^{r}$ submersion

$$
\pi: V \rightarrow S^{1}
$$

such that the identity

$$
\omega=f \pi^{*} \theta
$$

holds throughout $V$, where $f$ is some positive-valued function of class $C^{r-1}$ on $V$.

Proof. Obvious.

The following assertion is an immediate consequence of Theorem $I^{\prime}$.

COROLLARY 5.3.1. Under the same hypotheses as in Theorem I, the one-form $\omega$ can be written in the form

$$
\omega=f \alpha,
$$

where $f$ is a positive-valued function of class $C^{r-1}$, and $\alpha$ is a nonsingular, closed one-form of class $C^{r-1}$.

Next, we consider the relation of the homology class of $\Sigma$ and the Euler characteristics of leaves.

Proposition 5.3.2. Let $S^{1}, \theta$ be as above. Suppose that $(V, \omega)$ has 
the following property: there exists a $C^{1}$ submersion $\pi: V \rightarrow S^{1}$ such that the identity $\omega=f \pi^{*} \theta$ holds throughout $V$, where $f$ is a positive-valued function. Then for $b \in S^{1}$, the Euler characteristic of the closed $n$-manifold $\pi^{-1}(b)$ is equal to the integral of $\pi^{*} \theta$ on the critical cycle $\Sigma$ (with the orientation given in 5.2).

Proof. Denote by $\pi_{1}$ the restriction of $\pi$ to $\Sigma$. Let $b \in S^{1}$. We may assume that the map $\pi_{1}: \Sigma \rightarrow S^{1}$ is transversal to $b$. Because, the set of regular values of $\pi_{1}$ is dense in $S^{1}$, and for any $b^{\prime} \in S^{1}$, the two manifolds $\pi^{-1}(b), \pi^{-1}\left(b^{\prime}\right)$ are diffeomorphic and hence their Euler characteristics are equal. Note that $\pi^{-1}(b) \cap \Sigma_{*}=\emptyset$. We denote by deg $\pi_{1}$ the degree of the mapping $\pi_{1}$ between the oriented manifolds $\Sigma, S^{1}$. It is easy to verify that the integral of $\pi^{*} \theta$ on $\Sigma$ is equal to $\operatorname{deg} \pi_{1}$. On the other hand, let $m_{+}$(or $m_{-}$) be the number of points $p$ in $\pi_{1}^{-1}(b)$ such that

$$
\theta_{b}\left(\pi_{1 *} \xi_{2}(p)\right)>0 \quad\left(\text { or } \theta_{b}\left(\pi_{1 *} \xi_{2}(p)\right)<0\right),
$$

where $\pi_{1^{*}}: T_{p}(\Sigma) \rightarrow T_{b}\left(S^{1}\right)$ is the induced map, and $\xi_{2}(p)$ is to be understood as a nonzero vector in $T_{p}(\Sigma)$ which defines the orientation of $\Sigma$ at $p$. It is well-known that $\operatorname{deg} \pi_{1}=m_{+}-m_{-}$. We shall prove that the Euler characteristic of $\pi^{-1}(b)$ is equal to $m_{+}-m_{-}$. The following fact is essential: if a point $p \in \pi_{1}^{-1}(b)$ is of type $(i)$, for some $i=0, \cdots, n$, then

$$
(-1)^{i} \theta_{b}\left(\pi_{1 *} \xi_{2}(p)\right)>0 \text {. }
$$

This fact can be checked directly. For $i=0, \cdots, n$, we denote by $c_{i}$ the number of elements in the finite set $\pi^{-1}(b) \cap \Sigma_{i}$. From the fact that for $i=0, \cdots, n$ and a point $p \in \pi^{-1}(b) \cap \Sigma_{i}$, the index of the closed one-form $\omega^{\prime} \mid \pi^{-1}(b)$ at the singular point $p$ is equal to $i$, we know that the Euler characteristic of $\pi^{-1}(b)$ is equal to $\sum_{i=0}^{n}(-1)^{i} c_{i}$. From the relation

$$
m_{+}=\sum_{i: \text { even }} c_{i}, \quad m_{-}=\sum_{i: \text { odd }} c_{i},
$$

we conclude that the Euler characteristic of $\pi^{-1}(b)$ is equal to $m_{+}-m_{-}$. This proves our proposition.

The next corollary is an immediate consequence of Theorem I, Theorem $\mathrm{I}^{\prime}$ and Proposition 5.3.2.

COROLlary 5.3.3. Let the hypotheses be as in Theorem I. Let $\nu$ be a vector field of class $C^{1}$ on $V$ such that $\omega_{x}\left(\nu_{x}\right) \neq 0$ for all $x \in V$. Denote by $[\Sigma]$ the integral homology class of $\Sigma$ (with the orientation defined in 
5.2), in $H_{1}(V ; Z)$. If $[\Sigma] \neq 0$, then there exists a closed integral curve of $\nu$.

\section{§6. Proof of Theorem II.}

Let $\mathfrak{g}, X,\left\{\varphi_{s}\right\}, Y, \omega^{\prime}$ be as in $\S 5$.

6.1. Notations and a lemma. For a connected, compact $(n-1)$-manifold $G$ of $V$, with or without boundary, which has a Riemannian structure induced by $g$, we denote by $\operatorname{diam}_{G}(G)$ the diameter of the Riemannian manifold $G$, i.e., the maximum distance in $G$ between any pairs of points of $G$, and we denote by $\operatorname{mes}_{n-1}(G)$ the total measure of $G$ with respect to the Riemannian measure on $G$. Similarly, for an open subset $G$ of a compact $(n-1)$-submanifold of $V$ or for an open subset $H$ of a compact $n$-submanifold of $V$, we can define $\operatorname{mes}_{n-1}(G)$ or $\operatorname{mes}_{n}(H)$, and we can also define $\operatorname{diam}_{G}(G)$ if $G$ is connected. For an open or closed subset $C$ of $V$, let $\operatorname{mes}_{n+1}(C)$ denote the measure of $C$ with respect to the Riemannian measure on $V$.

For a subset $G$ of a vein of $(V, \omega)$ and a number $u \geq 0$, we denote by $G[u]$ the subset of $V$ consisting of those points $x$ such that there exists an admissible tangential curve $a:[0, \tau] \rightarrow V$ satisfying the following conditions :

$$
\mathfrak{a}(0) \in G ; \quad \mathfrak{a}(\tau)=x ; \quad \int_{\mathrm{a}[0, \tau]} \omega^{\prime}=-u .
$$

For a vein $J$ and positive numbers $\sigma, \mu$, we define

$$
\operatorname{cub}(J, \sigma, \mu)=\bigcup_{s=0}^{\sigma} \varphi_{s}\left(\bigcup_{u=1}^{\mu} J[u]\right) .
$$

LEMMA 6.1.1. If $\Sigma_{0}=\emptyset$, and $\Sigma_{n-1}=\emptyset$, then there exist three positive constants $c_{*}, d$, e such that the inequality

$$
c_{*} \cdot \sigma \cdot \mu \cdot \operatorname{diam}_{J}(J) \leq \operatorname{mes}_{n+1}(\operatorname{cub}(J, \sigma, \mu))
$$

holds for any numbers $\sigma, \mu$ satisfying $0<\sigma \leq 1,0<\mu \leq e$, and for any nonsingular compact vein $J$ satisfying the following conditions:

$$
\begin{aligned}
& \operatorname{diam}_{J}(J)>d ; \\
& J \cap J[u]=\emptyset \quad \text { for } 0<u \leq \mu ; \\
& \left(\bigcup_{u=0}^{\mu} J[u]\right) \cap \varphi_{s}\left(\bigcup_{u=0}^{\mu} J[u]\right)=\emptyset \quad \text { for } 0<s \leq \sigma .
\end{aligned}
$$


This lemma will be proved in 6.4.

6.2. Proof of Theorem II. The proof will be preceded by five lemmas, 6.2.1-6.2.5.

LeMma 6.2.1. Suppose that $\Sigma_{n} \neq \emptyset, \Sigma_{n-1}=\emptyset, \Sigma_{0}=\emptyset$. Let $p$ be a point in $\Sigma_{n}$, and $S^{1}(p)$ the connected component of $\Sigma$ containing $p$. Put

$$
R=\bigcup_{q \in S^{1}(p)} L(q),
$$

where $L(q)$ is a leaf through a point $q$. Then:

(i) Every point in $S^{1}(p)$ is of type $(n)$.

(ii) $R$ is a connected, noncompact, open subset in $V$.

Further, there exists a $C^{r-1}$ submersion $\pi$ of $R$ onto $S^{1}(p)$ having the following properties:

(iii) The restriction $\pi \mid S^{1}(p)$ is the identity.

(iv) For each $q \in S^{1}(p)$, the $n$-submanifold $\pi^{-1}(q)$ is a noncompact, simply connected leaf of $(V, \omega)$.

(v) For each $q \in S^{1}(p)$, there exists an open set I in $S^{1}(p)$ containing $q$ and $a C^{r-1}$ diffeomorphism

$$
\Phi: I \times \pi^{-1}(q) \rightarrow \pi^{-1}(I)
$$

such that $\pi(\Phi(y, x))=y$ for all $y \in I$ and all $x \in \pi^{-1}(q)$, and such that for each point $x_{0} \in \pi^{-1}(q)$, there exists a $C^{r-1}$ imbedding $\theta: I \rightarrow \boldsymbol{R}$ satisfying

$$
\Phi\left(y, x_{0}\right)=\varphi_{\theta(y)}\left(x_{0}\right) \quad \text { for all } y \in I \text {. }
$$

Furthermore, for any point $x$ of $R$ and any real number $\eta$ satisfying

$$
\varphi_{s \eta}(x) \in R \quad \text { for all } s \in[0,1],
$$

there exists a $C^{r}$ diffeomorphism

$$
\Lambda_{x, \eta}: L(x) \rightarrow L\left(\varphi_{\eta}(x)\right),
$$

where $L(x), L\left(\varphi_{\eta}(x)\right)$ are leaves through $x, \varphi_{\eta}(x)$, respectively, such that the following condition is satisfied: there exists a $C^{r}$ function $\lambda: L(x) \rightarrow$ $\boldsymbol{R}$ satisfying

$$
\lambda(x)=\eta, \Lambda_{x, \eta}(y)=\varphi_{\lambda(y)}(y) \quad \text { for all } y \in L(x) .
$$

Proof. Part (i) follows from the assumption $\Sigma_{n-1}=\emptyset$ and the fact that $\Sigma$ is covered by $\mathscr{F}$-charts of "type $(\lambda)$ " or of "type $(*, \mu)$ " (see 4.1 ). 
Note that (i) implies that the one-dimensional sphere $S^{1}(p)$ of class $C^{r-1}$ is transversal to leaves.

Assertion 1. Suppose that a leaf through a point $q$ of $S^{1}(p)$ does not intersect $\Sigma_{*}$. Then $L$ is simply connected and noncompact, and satisfies $L \cap \Sigma_{n}=q$.

Proof. From Remark 2.2.1 we know that the closed one-form $-\omega^{\prime} \mid L$ on $L$ is proper. For $i=0,1, \cdots, n$ and a point $x$ of $L$, note the following fact: $x$ belongs to $\Sigma_{i}$ if and only if $x$ is a nondegenerate singular point of $-\omega^{\prime} \mid L$, with index $(n-i)$. Hence, by the assumption $\Sigma_{n-1}=\emptyset$, we can apply Proposition 2.2.1 to $\left(L,-\omega^{\prime} \mid L\right)$. Assertion 1 follows from (ii), (iii) and (iv) of Proposition 2.2.1.

Assertion 2. There exists a positive number $\rho$ having the following properties:

(i) $|\rho|<\eta_{*}$, where $\eta_{*}$ is the same constant as in Lemma 4.1.3;

(ii) for each $q \in S^{1}(p)$, a mapping defined by $s \mapsto \varphi_{s}(q)$ of $(-\rho, \rho)$ into $V$, is one-to-one;

(iii) for each $q \in S^{1}(p)$, there exists an imbedding $l:(-\rho, \rho) \rightarrow S^{1}(p)$ of class $C^{r-1}$ such that for any $s \in(-\rho, \rho)$, the leaf through $\varphi_{s}(q)$ passes through $l(s)$.

Proof. Since $S^{1}(p)$ is a one-dimensional sphere of class $C^{r-1}$, and is transversal to leaves, using the facts that for a point $q$ of $V$, there exists an $\mathscr{F}$-chart at $q$ of class $C^{r}$, and the curve $\varphi_{s}(q)(s \in R)$ is of class $C^{r}$ ( $X$ is assumed to be of class $C^{r}$ ), one easily verifies Assertion 2.

Assertion 3. Let $\rho$ be as in Assertion 2. Let $q \in S^{1}(p)$. If a tangential curve $\mathrm{c}:[0, \tau] \rightarrow V$ satisfies

$$
\mathfrak{c}(0)=\varphi_{s}(q), \mathfrak{c}(\tau)=\varphi_{s^{\prime}}(q) \quad \text { for some } s, s^{\prime} \in(-\rho, \rho),
$$

then $\mathfrak{c}(0)=\mathfrak{c}(\tau)$, and $s=s^{\prime}$.

Proof. Suppose that $c(0) \neq c(\tau)$. Recall that for almost every $\eta \in(-\rho, \rho)$, the leaf through $\varphi_{\eta}(q)$ does not intersect $\Sigma_{*}$. Then, considering a suitable lift of $c$, we should find a leaf which does not intersect $\Sigma_{*}$, and passes through two distinct points $\varphi_{\eta}(q), \varphi_{\eta^{\prime}}(q), \eta, \eta^{\prime} \in(-\rho, \rho)$. By (iii) of Assertion 2, such a leaf would intersect at least two points of 
$\Sigma_{n}$, which contradicts Assertion 1. Hence $c(0)=\mathfrak{c}(\tau)$. Part (ii) of Assertion 2 implies $s=s^{\prime}$.

Assertion 4. Suppose that a leaf $L$ through a point $q$ of $S^{1}(p)$ does not intersect $\Sigma_{*}$. Let $\rho, l$ be as in Assertion 2. Then there exists a $C^{r}$ imbedding

$$
\Psi:(-\rho, \rho) \times L \rightarrow R
$$

such that for each $s \in(-\rho, \rho)$, the image $\Psi(s \times L)$ is a leaf through $l(s)$.

Proof. As in the proof of Assertion 1, we can apply Proposition 2.2.1 to $\left(L,-\omega^{\prime} \mid L\right)$. For $(\eta, x) \in(-\rho, \rho) \times L$, let $a:[0, \tau] \rightarrow V$ be an admissible tangential curve such that $\mathfrak{a}(0)=q, \mathfrak{a}(\tau)=x$. From Lemma 4.1.3 we know that $a$ has the $\eta$-lift with height parameter $\sigma$. Then define

$$
\Psi(\eta, x)=\varphi_{\sigma(\tau)}(x)
$$

From Assertion 3 we observe that $\Psi(\eta, x)$ is defined independently of the choice of admissible tangential curves $a$, and that $\Psi$ is one-to-one. Hence, it is obvious that $\Psi$ is an imbedding. Using the fact that the height parameters $\sigma$ of the $\eta$-lifts $(|\eta|<\rho)$ of admissible tangential curves $\mathfrak{a}:[0, \tau] \rightarrow V$ are bounded, indeed, we have, by Lemma 4.1.3,

$$
|\sigma(t)|<\rho /\left(1-\rho \beta_{*}\right),
$$

where $\beta_{*}$ is as in Lemma 4.1.3, we know that for each $\eta \in(-\rho, \rho)$, the image $\Psi(\eta \times L)$ coincides with a leaf, which passes through $l(\eta)$. That $\Psi$ is of class $C^{r}$ follows from the facts that for every point $x$ of $V$, there exists an $\mathscr{F}$-chart at $x$ of class $C^{r}$, and the curve $\varphi_{s}(x)(s \in \boldsymbol{R})$ is of class $C^{r}$. Thus $\Psi$ satisfies our requirements.

We can now prove (ii)-(v) of our lemma. First, for $x \in R$, we define $\pi(x)$ to be a point where the leaf through $x$ intersects $S^{1}(p)$. The similar argument to the proof of Assertion 3 shows that the map $\pi: R \rightarrow S^{1}(p)$ is well-defined. By definition, each inverse image $\pi^{-1}(q), q \in S^{1}(p)$, is a leaf. That the map $\pi$ is of class $C^{r-1}$ follows from the fact that the one-sphere $S^{1}(p)$ is of class $C^{r-1}$. It is obvious that the restriction $\pi \mid S^{1}(p)$ is the identity, and therefore that $\pi$ is a submersion. Now, let $\rho$ be as in Assertion 2, and let $q^{\prime}$ be a point of $S^{1}(p)$. Then we can find $L, q, l, \Psi$ as in Assertion 4, such that $q^{\prime} \in \operatorname{Im} \Psi$, because for almost every $q \in S^{1}(p)$, the leaf through $q$ does not intersect $\Sigma_{*}$. Hence $\pi^{-1}\left(q^{\prime}\right)$ is diffeomorphic 
to $L$, and thus $\pi^{-1}\left(q^{\prime}\right)$ is noncompact and simply connected. Furthermore, putting $I=\operatorname{Im} l$, and using the maps $\Psi, l$, one obtain the required diffeomorphism $\Phi: I \times \pi^{-1}\left(q^{\prime}\right) \rightarrow \pi^{-1}(I)$ to prove (v). Our last assertion is an immediate consequence of (v). This completes the proof of Lemma 6.2.1.

Remark 6.2.1. Let the hypotheses and the notation be as in Lemma 6.2.1. Let $S^{1}$ be the ordinary one-dimensional sphere. Then there exists a $C^{r}$ submersion $\tilde{\pi}$ of $R$ onto $S^{1}$ having the similar properties to $\pi$. More precisely, $\tilde{\pi}$ satisfies the following conditions:

(i) The restriction $\tilde{\pi} \mid S^{1}(p): S^{1}(p) \rightarrow S^{1}$ is a diffeomorphism.

(ii) Each inverse image $\tilde{\pi}^{-1}(q)$ is a noncompact, simply connected leaf.

(iii) For each $q \in S^{1}$, there exists an open set $I$ in $S^{1}$ containing $q$ and a $C^{r}$ diffeomorphism

$$
\Phi: I \times \tilde{\pi}^{-1}(q) \rightarrow \tilde{\pi}^{-1}(I)
$$

such that $\tilde{\pi}(\Phi(y, x))=y$ for all $y \in I$ and all $x \in \tilde{\pi}^{-1}(q)$.

This fact may be seen as follows. Since $S^{1}(p)$ is a one-dimensional sphere of class $C^{r-1}$ which is transversal to leaves, smoothing this imbedded one-sphere, we have a $C^{r+1}$ imbedding

$$
i: S^{1} \rightarrow V^{n+1}
$$

such that the composition $\pi \circ i: S^{1} \rightarrow S^{1}(p)$ is a diffeomorphism. It is easy to verify that a map $\tilde{\pi}$ defined by

$$
\tilde{\pi}(x)=(\pi \circ i)^{-1}(\pi(x)), \quad x \in R,
$$

gives us the required submersion.

LEMMA 6.2.2. Let the hypotheses and the notation be as in Lemma 6.2.1. Let $L$ be a leaf which is contained in $R$ and does not intersect $\Sigma_{*}$. Let $f: L \rightarrow \boldsymbol{R}$ be a first integral of $\omega^{\prime} \mid L$, i.e., satisfy

$$
d f=\omega^{\prime} \mid L
$$

(Such a function does exist by (i) of Proposition 2.2.1.) Let $v$ be a real number. Assume that $f^{-1}((-\infty, v]) \cap \Sigma_{1}=\emptyset$. Suppose that $f^{-1}(v)$ is a finite union of nonsingular, compact veins $J_{j}^{n-1}, j=1, \cdots, m$, and has a "nice" fence in the following sense: There exists an immersion 


$$
F: f^{-1}(v) \times[0, \varepsilon] \rightarrow V
$$

such that

(i) the restriction $F \mid f^{-1}(v) \times 0$ is the identify;

(ii) for each $s \in[0, \varepsilon]$ and each $j=1, \cdots, m$, the image $F\left(J_{j} \times s\right)$ is contained in a leaf, and if $s \neq \varepsilon$, the restriction $F \mid J_{j} \times s$ is an imbedding;

(iii) the image $F\left(f^{-1}(v) \times[0, \varepsilon)\right)$ is contained in $R$;

(iv) the image $F\left(f^{-1}(v) \times \varepsilon\right)$ does not contained in $R$;

(v) for each $x \in f^{-1}(v)$, the curve $c:[0, \varepsilon] \rightarrow V$ defined by $c(s)=$ $F(x, s)$ can be expressed in the form

$$
c(s)=\varphi_{\rho(s)}(x), \quad s \in[0, \varepsilon],
$$

for a suitable imbedding $\rho:[0, \varepsilon] \rightarrow \boldsymbol{R}$ satisfying

$$
\rho(0)=0, \quad|\rho(\varepsilon)|<\eta_{*},
$$

where $\eta_{*}$ is the same constant as in Lemma 4.1.3. Then for each $J_{j}$, the leaf containing $F\left(J_{j} \times \varepsilon\right)$ is compact.

Proof. We shall prove that the leaf containing $F\left(J_{1} \times \varepsilon\right)$ is compact.

Assertion 1. There exists an infinite sequence $s_{1}, s_{2}, \ldots$ of points in $[0, \varepsilon)$ such that $\lim _{i \rightarrow \infty} s_{i}=\varepsilon$, and for each $s_{i}$, the set $F\left(J_{1} \times s_{i}\right)$ is contained in $L$.

To prove this, fix a point $x_{1}$ of $J_{1}$. By assumption, a curve $c_{1}:[0, \varepsilon]$ $\rightarrow V$ defined by $c_{1}(s)=F\left(x_{1}, s\right)$, is transversal to leaves. Hence, for $\pi: R \rightarrow S^{1}(p)$ as in Lemma 6.2.1, the composition

$$
\pi \circ c_{1}:[0, \varepsilon) \rightarrow S^{1}(p)
$$

is a submersion. Since $c_{1}(\varepsilon) \notin R$, we observe that $\lim _{s \rightarrow \varepsilon-0} \pi \circ c_{1}(s)$ does not exist, and hence that for a point $q=\pi(L)$, the set $\left(\pi \circ c_{1}\right)^{-1}(q)$ is infinite. Since $\left(\pi \circ c_{1}\right)^{-1}(q)$ does not have the accumulating point in $[0, \varepsilon)$, there exists an infinite sequence $s_{1}, s_{2}, \cdots, s_{i} \rightarrow \varepsilon$, such that $c_{1}\left(s_{1}\right), c_{1}\left(s_{2}\right), \cdots \in L$. From the condition (ii) of $F$, it follows that $F\left(J_{1} \times s_{i}\right) \subset L$ for all $i$.

Assertion 2. For any real number $w$ and any $J_{j}$, there exists $\delta \in(0, \varepsilon)$ such that if $F\left(J_{j} \times s\right) \subset L$ for some $s \in(\delta, \varepsilon)$, then

$$
F\left(J_{j} \times s\right) \subset f^{-1}((-\infty, w]) .
$$

To prove this, fix a point $x$ of $J_{j}$, and consider the subset 


$$
F(x \times[0, \varepsilon]) \cap L
$$

of $L$. It is clear that any sequence of points in this set does not converge in $L$. Since $f([w-1, \infty))$ is compact (by (i) and (v) of Proposition 2.2.1), the subset of $F(x \times[0, \varepsilon]) \cap L$ consisting of those points $y$ such that $f(y) \geq w-1$, is finite. Hence there exists $\delta_{x} \in(0, \varepsilon)$ such that, if $F(x, s) \in L$ for some $s \in\left(\delta_{x}, \varepsilon\right)$, then

$$
f(F(x, s))<w-1 .
$$

Using the fact that there exists an $\mathscr{F}$-chart whose coordinate neighborhood contains the set $F(x \times[0, \varepsilon])$, we see that for a sufficiently small neighbourhood $U_{x}$ of $x$ in $J_{j}$, the inequality

$$
|f(F(y, s))-f(F(x, s))|<1
$$

holds for any $y \in U_{x}$ and any $s \in[0, \varepsilon)$ satisfying $F(x, s) \in L$. Consequently, the number $\delta_{x}$ has the following property: if $F(x, s) \in L$ for some $s \in\left(\delta_{x}, \varepsilon\right)$, then $f(F(y, s))<w$ for all $y \in U_{x}$, that is,

$$
F\left(U_{x} \times s\right) \subset f^{-1}((-\infty, w)) .
$$

Since $J_{j}$ is compact, we can find $\delta \in(0, \varepsilon)$ with the desired property.

Now, for each $j=1, \cdots, m$, fix a point $x_{j} \in J_{j}$, and let $c_{j}, \rho_{j}$ be as in (v) of Lemma 6.2.2, i.e.,

$$
F\left(x_{j}, s\right)=c_{j}(s)=\varphi_{\rho_{j}(s)}\left(x_{j}\right), \quad s \in[0, \varepsilon] .
$$

Suppose for definiteness that $\rho_{1}(\varepsilon)>0$. We may assume that for some integer $l \geq 1$,

$$
\begin{array}{ll}
\rho_{i}(\varepsilon)>0 & \text { for } 1 \leq i \leq l, \\
\rho_{i}(\varepsilon)<0 & \text { for } l+1 \leq i \leq m .
\end{array}
$$

The following is essential to our argument.

Assertion 3. There exists a compact $n$-submanifold $W$ in $L$ having the following properties:

(i) the boundary $\partial W$ coincides with the union of

$$
J_{1}, \cdots, J_{l}, \quad \text { and } F\left(J_{1} \times r_{1}\right), \cdots, F\left(J_{l} \times r_{l}\right) \text {, }
$$

where $r_{1}, \cdots, r_{l}$ are suitable numbers in $(0, \varepsilon)$;

(ii) for any point $y$ of $W$, there exists an admissible tangential curve starting at some point of $\bigcup_{i=1}^{l} J_{i}$, with the terminal point $y$; 
(iii) for two admissible tangential curves $\mathfrak{a}_{i}:\left[0, \tau_{i}\right] \rightarrow V, i=1,2$, with $\mathfrak{a}_{1}\left(\tau_{1}\right)=\mathfrak{a}_{2}\left(\tau_{2}\right) \in W$, if $\mathfrak{a}_{1}(0) \in J_{i_{1}}$ and $\mathfrak{a}_{2}(0) \in J_{i_{2}}$, then $i_{1}=i_{2}$;

(iv). for any point $x$ of $J_{j}, j=1, \cdots, l$, there exists a neighborhood $U$ of $x$ in $L$ and a neighborhood $U^{\prime}$ of $F\left(x, r_{j}\right)$ in $L$ such that the map

$$
\Lambda_{x, \rho\left(x_{j}\right)}: L \rightarrow L
$$

as in Lemma 6.2.1, induces a diffeomorphism of $U$ onto $U^{\prime}$, and maps $U \cap W$ onto $U^{\prime}-\left(U^{\prime} \cap(W-\partial W)\right)$, where $\rho$ is as in (v) of Lemma 6.2.2.

Proof. For the fixed point $x_{1} \in J_{1}$ and a number $s \in[0, \varepsilon)$, let

$$
\Lambda_{x_{1}, \rho_{1}(s)}: L \rightarrow L\left(F\left(x_{1}, s\right)\right)
$$

be as in Lemma 6.2.1. If $F\left(x_{1}, s\right) \in L$, we denote this map by $\Lambda^{s}$, for simplicity of notation. By Assertion 1, there is an infinite sequence $s_{1}, s_{2}, \cdots, s_{i} \rightarrow \varepsilon-0$, such that $F\left(J_{1} \times s_{i}\right) \subset L$. It is clear that for the base points $x_{j}, j=1, \cdots, m$, and any $i=1,2, \cdots$, the points $\Lambda^{s_{i}}\left(x_{j}\right)$ are contained in $L$, and that if $1 \leq j \leq l$, there are suitable $r_{j i} \in(0, \varepsilon)$ satisfying

$$
A^{s i}\left(x_{j}\right)=\varphi_{\rho_{j}\left(r_{j i}\right)}\left(x_{j}\right)=F\left(x_{j}, r_{j i}\right) .
$$

Note that for each $1 \leq j \leq l, r_{j i} \rightarrow \varepsilon-0$ as $i \rightarrow \infty$. From Assertion 2, it follows that for any real number $w$, there is an integer $i$ such that

$$
f(y)<w \quad \text { for all } y \in F\left(J_{j}, r_{j i}\right), j=1, \cdots, l .
$$

Hence, taking sufficiently large $i$, and putting $s_{*}=s_{i}$, we observe that $F\left(x_{1}, s_{*}\right) \in L$, and for any point $y$ of $\Lambda^{s_{*}}\left(J_{j}\right), j=1, \cdots, l$, there exists an admissible tangential curve starting at some point of $\bigcup_{i=1}^{l} J_{i}$, with the terminal point $y$. To prove this, suppose that there were any admissible tangential curve $\mathfrak{a}:[-1, \tau] \rightarrow V, \mathfrak{a}(-1) \in \Sigma_{n}$, such that

$$
\mathfrak{a}(\tau) \in \Lambda^{s_{*}}\left(J_{j}\right)(j=1, \cdots, l) \text {, and } \operatorname{Im} \mathfrak{a} \cap \bigcup_{i=1}^{l} J_{i}=\emptyset .
$$

Then the curve $\mathfrak{a}$ would intersect $\bigcup_{i=l+1}^{m} J_{i}$. We might assume $\mathfrak{a}(0) \in J_{k}$ $(k=l+1, \cdots, m)$. Since $\rho_{k}(\varepsilon)<0$, by (v) of Lemma 6.2 .2 , there would exist a real number $\eta$ such that

$$
-\eta_{*}<\eta<0 \text {, and } \varphi_{\eta}(\mathfrak{a}(0)) \notin R \text {. }
$$

Considering the $\eta$-lift of $\mathfrak{a}$, and applying Lemma 4.1.3, one would conclude that for the point $a(\tau) \in \Lambda^{s_{*}}\left(J_{j}\right)$ and some negative number $\sigma$ sufficiently 
close to $0, \varphi_{o}(\mathfrak{a}(\tau)) \notin R$. This contradicts the fact that there exists a curve $c:[0, \varepsilon] \rightarrow V$ which passes through $a(\tau)$, near the point $c(\varepsilon)$, and which is written in the form

$$
c(s)=\varphi_{\rho(s)}(x), \quad s \in[0, \varepsilon],
$$

where $x \in J_{j}$, and $\rho$ is as in (v) of Lemma 6.2.2, and $\rho(\varepsilon)>0$ (since $1 \leq j \leq l$ ). Now, in order to prove Assertion 3, consider the decomposition

$$
\begin{gathered}
L=f^{-1}([v, \infty)) \cup L_{1} \cup \cdots \cup L_{l} \cup L_{l+1} \cup \cdots \cup L_{m}, \\
L_{i} \cap L_{j}=\emptyset \quad \text { if } i \neq j,
\end{gathered}
$$

where each $L_{i}$ is a connected, noncompact $n$-submanifold in $L$, with boundary $\partial L_{i}=J_{i}$, such that

$$
f^{-1}([v, \infty)) \cap L_{i}=\partial L_{i},
$$

and such that for any $y \in L_{i}$, there is an admissible tangential curve starting at some point of $J_{i}$, with the terminal point $y$. (Such a decomposition exists, since $L$ is simply connected.) We put

$$
W=\left(L_{1} \cup \cdots \cup L_{l}\right) \cap \Lambda^{s *}\left(f^{-1}([v, \infty)) \cup L_{l+1} \cup \cdots \cup L_{m}\right),
$$

where $s_{*}$ is the number mentioned above. From the property of $s_{*}$, we know that for each $i=1, \cdots, l$, the manifold $\Lambda^{s_{*}}\left(J_{i}\right)$ is contained in some $L_{j}, j=1, \cdots, l$. Furthermore, for $i=1, \cdots, l$, if $\Lambda^{*}\left(J_{i}\right)$ is contained in $L_{j}$, then $\Lambda^{s_{*}}\left(L_{i}\right)$ is also contained in $L_{j}$. (If $\Lambda^{s_{*}}\left(L_{i}\right)$ were not contained in $L_{j}$, then $\Lambda^{s *}\left(L_{i}\right)$ would contain $L-L_{j}$. This contradicts the fact that there exists a point $p \in L-L_{j}$ such that $\left\{\varphi_{s}(p)\right\}_{s \geqslant 0} \subset R$ (Lemma 6.2.1).) From the assumption that $f^{-1}((-\infty, v]) \cap \Sigma_{1}=\emptyset$, it follows that for any $w \leq v$ and each $L_{i}$, the set $L_{i} \cap f^{-1}(w)$ is connected. Using these facts, we conclude that $W$ is a compact $n$-submanifold, with boundary

$$
\partial W=J_{1} \cup \cdots \cup J_{l} \cup \Lambda^{s *}\left(J_{1}\right) \cup \cdots \cup \Lambda^{s *}\left(J_{l}\right) .
$$

It is clear that each $\Lambda^{s_{*}}\left(J_{i}\right), i=1, \cdots, l$, can be expressed in the form $F\left(J_{i} \times r_{i}\right)$ for some $r_{i} \in(0, \varepsilon)$. Furthermore, one verifies that $W$ satisfies the desired properties (ii), (iii), (iv). This completes the proof of Assertion 3.

Now, in order to prove Lemma 6.2.2, for the manifold $W$ in Assertion 3, we define a map $\Lambda: W \rightarrow V$ as follows. For a point $y$ of $W$, let $a:[0, \tau] \rightarrow V$ be an admissible tangential curve such that 


$$
\mathfrak{a}(0) \in \bigcup_{i=1}^{l} J_{i}, \quad \mathfrak{a}(\tau)=y .
$$

By (iii),(v) of Lemma 6.2.1, there exists a real number $\eta$ such that

$$
\begin{aligned}
& 0<\eta<\eta_{*}, \\
& F(\mathfrak{a}(0), \varepsilon)=\varphi_{\eta}(\mathfrak{a}(0)), \\
& \varphi_{s \eta}(\mathfrak{a}(0)) \in R \quad \text { for } 0 \leq s<1 .
\end{aligned}
$$

Let $\mathfrak{b}:[0, \tau] \rightarrow V$ be the $\eta$-lift of $\mathfrak{a}$. Then we define $\Lambda(y)=\mathfrak{b}(\tau)$. Using (iii) of Assertion 3 and the fact that every leaf in $R$ is simply connected, one easily verifies that the point $\Lambda(y)$ is determined independently of the choice of admissible curves $\mathfrak{a}$ (cf. the proof of Lemma 6.2.3), and that this map $A$ is expressed locally in the following form: for each $y \in W$,

$$
\Lambda(z)=\varphi_{\lambda(z)}(z) \quad \text { for } z \in U,
$$

where $U$ is a neighborhood of $y$ in $W$, and $\lambda$ is a suitable positive-valued function defined on $U$. Furthermore, by (iii), (iv) of Lemma 6.2.2, for each $F\left(J_{i} \times r_{i}\right)$ in (i) of Assertion 3, the image $\Lambda\left(F\left(J_{i} \times r_{i}\right)\right)$ coincides with $F\left(J_{i} \times \varepsilon\right)$. Hence we see that $\Lambda$ is a submersion of $W$ into the leaves $K_{1}, \cdots, K_{k}(1 \leq k \leq l)$ containing the sets $F\left(J_{1} \times \varepsilon\right), \cdots, F\left(J_{l} \times \varepsilon\right)$. From this and (iv) of Assertion 3, it follows that $\Lambda$ maps $W$ onto $K_{1}, \cdots, K_{k}$, and hence that $K_{1}, \cdots, K_{k}$ are compact, as desired. Similarly, it is proved that the leaves containing $F\left(J_{i} \times \varepsilon\right), i=l+1, \cdots, m$, are compact. This completes the proof of Lemma 6.2.2.

Remark 6.2.2. With the hypotheses and notation of the preceding lemma, for each $J_{j}$, there exists a connected, compact $n$-submanifold $W_{j}$ in $L$ having the following properties:

(i) the boundary $\partial W_{j}$ consists of $J_{j}$ and $F\left(J_{j} \times r_{j}^{\prime}\right)$, where $r_{j}^{\prime}$ is a suitable number in $(0, \varepsilon)$;

(ii) there exists a function $\lambda: W_{j} \rightarrow \boldsymbol{R}$ such that $|\lambda(z)|<\eta_{*}$ for all $z \in J_{j}$, and such that the map $\Lambda_{j}: W_{j} \rightarrow V$ defined by

$$
\Lambda_{j}(z)=\varphi_{\lambda(z)}(z) \quad \text { for } z \in W_{j},
$$

is a submersion of $W_{j}$ onto the compact leaf containing $F\left(J_{j} \times \varepsilon\right)$.

To prove this, recall the proof of Assertion 3. For the map $\Lambda^{s_{*}}$, conisidering a suitable iteration $\left(\Lambda^{s *}\right)^{i}$, we have

$$
\left(\Lambda^{s_{*}}\right)^{i}\left(J_{j}\right) \subset L_{j}, \quad j=1, \cdots, l .
$$


Then we define $W_{j}$ to be the manifold bounded by $J_{j}$ and $\left(\Lambda^{s_{*}}\right)^{i}\left(J_{j}\right)$. It is essentially proved above that $W_{j}$ satisfies the required properties. Similarly, for $j=l+1, \cdots, m$, we find $W_{j}$ as above. Thus our assertion is proved.

LEMMA 6.2.3. Suppose that a nonsingular, compact vein $J^{n-1}, a$ point $x_{0}$ of $J$, and a real number $\eta$ satisfy the following condition: for any $x \in J$, there exists a tangential curve $\mathfrak{c}:[0, \tau] \rightarrow V$ which satisfies

$$
\mathfrak{c}(0)=x_{0}, \quad \mathfrak{c}(\tau)=x, \quad \operatorname{Im} \mathfrak{c} \subset J,
$$

and has the $\eta$-lift. If for any $s \in[0,1)$, the leaf through $\varphi_{s \eta}(x)$ is simply connected, then $J$ with the base point $x_{0}$ has the $\eta$-lift, i.e., there exists a function $\lambda: J \rightarrow \boldsymbol{R}$ with $\lambda\left(x_{0}\right)=\eta$, such that for any $x \in J$, the point $\varphi_{\lambda(x)}(x)$ is contained in the leaf through $\varphi_{\eta}\left(x_{0}\right)$.

Proof. Let $x \in J$, and let $c_{i}:\left[0, \tau_{i}\right] \rightarrow V, i=1,2$, be two tangential curve satisfying $x_{0}=\mathfrak{c}_{1}(0)=\mathfrak{c}_{2}(0), x=\mathfrak{c}_{1}\left(\tau_{1}\right)=\mathfrak{c}_{2}\left(\tau_{2}\right)$, and having the $\eta$-lifts with height parameters $\sigma_{i}$. We shall prove that $\sigma_{1}\left(\tau_{1}\right)=\sigma_{2}\left(\tau_{2}\right)$. Then, defining $\lambda$ by $\lambda(x)=\sigma_{1}\left(\tau_{1}\right)$, one will obtain the required function $\lambda$. Now, to prove $\sigma_{1}\left(\tau_{1}\right)=\sigma_{2}\left(\tau_{2}\right)$, we consider a curve $\mathfrak{c}_{12}:\left[0, \tau_{1}+\tau_{2}\right] \rightarrow V$ defined by

$$
\begin{aligned}
& \mathfrak{c}_{12}(t)=\mathfrak{c}_{1}(t) \quad \text { for } t \in\left[0, \tau_{1}\right], \\
& \mathfrak{c}_{12}(t)=\mathfrak{c}_{2}\left(\tau_{1}+\tau_{2}-t\right) \quad \text { for } t \in\left[\tau_{1}, \tau_{1}+\tau_{2}\right] .
\end{aligned}
$$

It is clear that $x_{0}=\mathfrak{c}_{12}(0)=\mathfrak{c}_{12}\left(\tau_{1}+\tau_{2}\right)$, and that $\mathfrak{c}_{12}$ is tangential and has the $\eta$-lift. For definiteness, we assume $\eta>0$. For each $h \in[0, \eta]$, let $\sigma_{h}:\left[0, \tau_{1}+\tau_{2}\right] \rightarrow \boldsymbol{R}$ be the height parameter of the $h$-lift of $c_{12}$. Now, say $\sigma_{1}\left(\tau_{1}\right)$ were smaller than $\sigma_{2}\left(\tau_{2}\right)$. Then for the map $H:[0, \eta] \rightarrow[0, \eta]$ defined by

$$
H(h)=\sigma_{h}\left(\tau_{1}+\tau_{2}\right),
$$

the point $H(\eta)$ would be smaller than $\eta$, and furthermore for any integer $i \geq 1, H^{i}(\eta)$ would be smaller than $H^{i-1}(\eta)$. Hence for the limit point $\eta_{0}=$ $\lim _{i \rightarrow \infty} H^{i}(\eta)$, the $\eta_{0}$-lift of $\mathfrak{c}_{12}$ would be closed and have a non-trivial holonomy, which contradicts the assumption that the leaf through $\varphi_{\eta_{0}}\left(x_{0}\right)$ is simply connected. This proves Lemma 6.2.3.

LEMMA 6.2.4. Let the hypotheses and the notation be as in Lemma 6.2.1. Let $\eta_{*}$ be as in Lemma 4.1.3. Let a point $z$ of $V$ and a real. number $\eta$ have the following properties:

(i) $|\eta|<\eta_{*}$; 
(ii) $z \notin R, \varphi_{s \eta}(z) \in R$ for $0<s \leq 1$;

(iii) the leaf $L$ through $\varphi_{\eta}(z)$ does not intersect $\Sigma_{*}$;

(iv) the set $f^{-1}((-\infty, v])$ does not intersect $\Sigma_{1}$, where $f: L \rightarrow \boldsymbol{R}$ is a first integral of $\omega^{\prime} \mid L$, and $v=f\left(\varphi_{\eta}(z)\right)$.

Then the leaf $L(z)$ through $z$ is compact.

Proof. Let $\eta_{*}, \beta_{*}$ be the same constants as in Lemma 4.1.3. For simplicity, we assume $\eta<0$. The proof in the case $\eta>0$ is similar. Now, by Lemma 4.1 .4 we have an admissible tangential curve $a:[0, \infty)$ $\rightarrow V$ with $a(0)=\varphi_{\eta}(z)$. Put $\delta=-\eta$, for simplicity of notation. Clearly $z=\varphi_{\delta}(\mathfrak{a}(0))$. Let $\sigma$ be the height parameter of the $\delta$-lift of $a$. Let $J_{t}$ be the vein through the point $\mathfrak{a}(t)$. For any $t \in[0, \infty)$, by definition, we know that $J_{t}$ is a connected component of $f^{-1}\left(v_{t}\right)$, where

$$
v_{t}=v+\int_{a[0, t]} \omega^{\prime} .
$$

From (i) of Proposition 2.2.1, we see that $J_{t}$ is compact, and for almost every $t \in[0, \infty)$, the vein $J_{t}$ is nonsingular. The following is important.

Assertion 1. There exists a positive number $\tilde{\theta}$ such that for almost every $\theta \in[\tilde{\theta}, \infty)$, the vein $J_{\theta}$ is nonsingular and satisfies

$$
\sigma(\theta)<1 /\left(\kappa \operatorname{diam}_{J_{\theta}}\left(J_{\theta}\right)+\eta_{*}^{-1}\right),
$$

where $\kappa$ is the same positive number as in Corollary 3.1.2.

Proof. Put $q=\pi(\mathfrak{a}(0))$, and let $I, \Phi$ be as in (v) of Lemma 6.2.1. For the vein $J_{t}$ and a nonnegative number $u$, the set $J_{t}[u]$, by definition (6.1), is contained in $f^{-1}\left(v_{t}-u\right)$. Since $f^{-1}((-\infty, v]) \cap \Sigma_{1}=\emptyset$, it follows that $J_{t}[u]$ coincides with a connected component of $f^{-1}\left(v_{t}-u\right)$. Now, let $e$ be the same constant as in Lemma 6.1.1. Since the set $\bigcup_{u=0}^{e} J_{0}[u]$ is compact, we can find a positive number $\rho_{0}$ such that the set

$$
\operatorname{cub}\left(J_{0}, \rho_{0}, e\right)=\bigcup_{s=0}^{\rho_{0}} \varphi_{s}\left(\bigcup_{u=0}^{e} J_{0}[u]\right)
$$

is contained in $\pi^{-1}(I)$. It is clear that $\rho_{0}<\delta=-\eta<\eta_{*}$. For $t \in[0, \infty)$, consider the set

where

$$
\operatorname{cub}\left(J_{t}, \rho(t), e\right)=\bigcup_{s=0}^{\rho(t)} \varphi_{s}\left(\bigcup_{u=0}^{e} J_{t}[u]\right)
$$




$$
\rho(t)=\left(\rho_{0} \exp \int_{a[0, t]} \omega^{\prime}\right) /\left(1+\rho_{0} \beta_{*}\right) .
$$

Then, we contend that

$$
\operatorname{cub}\left(J_{t}, \rho(t), e\right) \subset \pi^{-1}(I) \quad \text { for all } t \in[0, \infty) .
$$

This is proved as follows. By (v) of Proposition 2.2.1, any point of the set on the left can be written as

$$
x=\varphi_{s}\left(\mathfrak{a}^{\prime}\left(t^{\prime}\right)\right),
$$

where $0 \leq s \leq \rho(t)$, and where $\mathfrak{a}^{\prime}:\left[0, t^{\prime}\right] \rightarrow V$ is an admissible tangential curve such that

$$
a^{\prime}(0) \in J[u] \quad \text { for some } u \in[0, e] \text {, and } \int_{a^{\prime}\left[0, t^{\prime}\right]} \omega^{\prime}=\int_{a[0, t]} \omega^{\prime} .
$$

By Lemma 4.1.3, there exists the $\rho_{0}$-lift of $\mathfrak{a}^{\prime}$, whose height parameter $\sigma^{\prime}$ satisfies

$$
0<\rho\left(t^{\prime}\right)<\sigma^{\prime}\left(t^{\prime}\right) .
$$

From this, we conclude that the point $x$ is contained in a leaf through some $\varphi_{s}\left(\mathfrak{a}^{\prime}(0)\right)$ with $s \in\left[0, \rho_{0}\right]$, and hence that $x \in \pi^{-1}(I)$, as desired. From the relation proved above and (v) of Lemma 6.2.1, it follows that

$$
\operatorname{cub}\left(J_{t}, \rho(t), e\right) \cap \operatorname{cub}\left(J_{t^{\prime}}, \rho\left(t^{\prime}\right), e\right)=\emptyset
$$

for $t, t^{\prime} \in[0, \infty)$ such that

$$
t<t^{\prime}, \text { and } \int_{a\left[t, t^{\prime}\right]} \omega^{\prime}<-e .
$$

Thus, since $V$ is compact, we have

$$
\operatorname{mes}_{n+1}\left(\operatorname{cub}\left(J_{t}, \rho(t), e\right)\right) \rightarrow 0 \text { as } t \rightarrow \infty,
$$

because, by Lemma 4.1.2, we have

$$
\int_{\mathrm{a}[0, t]} \omega^{\prime} \rightarrow-\infty \quad \text { as } t \rightarrow \infty .
$$

On the other hand, for any $t \in[0, \infty)$ it is clear that

$$
\begin{gathered}
\left.0<\rho(t)<1 \quad \text { (since } 0<\rho_{0}<\eta_{*}<1\right), \\
J_{t} \cap J_{t}[u]=\emptyset \quad \text { if } u>0,
\end{gathered}
$$

and that 


$$
\left(\bigcup_{u=0}^{e} J_{t}[u]\right) \cap \varphi_{s}\left(\bigcup_{u=0}^{e} J_{t}[u]\right)=\emptyset \quad \text { if } 0<s \leq \rho(t) .
$$

For simplicity, we assume that

$$
\operatorname{diam}_{J_{t}}\left(J_{t}\right)>d \text { for any } t \text { sufficiently large , }
$$

where $d$ is the same constant as in Lemma 6.1.1. In the general case, using the fact that by Lemmas 4.1.2 and 4.1.3,

$$
\sigma(t) \rightarrow 0 \quad \text { as } t \rightarrow \infty,
$$

one will prove Assertion 1 similarly. Thus we may apply Lemma 6.1.1 to obtain

$$
c_{*} \cdot \rho(t) \cdot e \cdot \operatorname{diam}_{J_{t}}\left(J_{t}\right) \leq \operatorname{mes}_{n+1}\left(\operatorname{cub}\left(J_{t}, \rho(t), e\right)\right),
$$

where $c_{*}$ is as in Lemma 6.1.1. Consequently, we have

$$
c_{*} \cdot \rho(t) \cdot e \cdot \operatorname{diam}_{J_{t}}\left(J_{t}\right) \rightarrow 0 \quad \text { as } t \rightarrow \infty \text {. }
$$

Recall that the height parameter $\sigma$ of the $\delta$-lift of $a$ satisfies

$$
0<\sigma(t)<\sigma_{*}^{+}(t) \quad \text { for all } t \in[0, \infty),
$$

where

$$
\sigma_{*}^{+}(t)=\left(\delta \exp \int_{\mathrm{a}[0, t]} \omega^{\prime}\right) /\left(1-\delta \beta_{*}\right)
$$

Note that

$$
\rho(t) / \sigma_{*}^{+}(t)=\rho_{0}\left(1-\delta \beta_{*}\right) / \delta\left(1+\rho_{0} \beta_{*}\right),
$$

which is constant. Hence we have

$$
\sigma_{*}^{+}(t) \cdot \operatorname{diam}_{J_{t}}\left(J_{t}\right) \rightarrow 0 \quad \text { as } t \rightarrow \infty,
$$

and thus

$$
\sigma(t) \cdot \operatorname{diam}_{J_{t}}\left(J_{t}\right) \rightarrow 0 \quad \text { as } t \rightarrow \infty .
$$

Using the fact $\sigma(t) \rightarrow 0(t \rightarrow \infty)$ again, we obtain

$$
\sigma(t)\left(\kappa \operatorname{diam}_{J_{t}}\left(J_{t}\right)+\eta_{*}^{-1}\right) \rightarrow 0 \quad \text { as } t \rightarrow \infty,
$$

which in particular proves Assertion 1.

Assertion 2. Let $\theta$ be as in Assertion 1. Then there exists an immersion

$$
F: J_{\theta} \times[0, \varepsilon] \rightarrow V
$$


which satisfies the same conditions (i)-(v) as in Lemma 6.2.2, such that the image $F\left(J_{\theta} \times \varepsilon\right)$ is contained in the leaf $L(z)$ through $z$.

Proof. It is clear that the point $\mathfrak{a}(\theta)$ and the number $\sigma(\theta)$ have the following properties:

$$
\begin{aligned}
& \varphi_{s \sigma(\theta)}(\mathfrak{a}(\theta)) \in R \quad \text { for } 0 \leq s<1 ; \\
& \varphi_{o(\theta)}(\mathfrak{a}(\theta)) \in L(z) .
\end{aligned}
$$

For any $x \in J_{\theta}$, by definition there exists a $C^{1}$ curve $\mathfrak{c}:[0, \tau] \rightarrow V$ such that

$$
\mathfrak{a}(\theta)=\mathfrak{c}(0) ; \quad x=\mathfrak{c}(\tau) ; \quad \operatorname{Im} \mathfrak{c} \subset J_{\theta} ; \quad\|\mathfrak{\mathfrak { c }}(t)\|=1 \quad \text { for } t \in[0, \tau],
$$

and such that the length of $\mathfrak{c}$, which is equal to $\tau$, is smaller than or equal to $\operatorname{diam}_{J_{\theta}}\left(J_{\theta}\right)$. Then, by Corollary 3.1.2 the tangential curve $c$ has the $\sigma(\theta)$-lift. Furthermore, from Lemma 3.1.1 (and the proof of Corollary 3.1.2) we know that its height parameter is smaller than or equal to $\sigma(\theta) /(1-\kappa \cdot \sigma(\theta) \cdot \tau)$, which is smaller than $\eta_{*}$, because our assumption concerning $\theta$ implies

$$
\eta_{*}^{-1}<(\sigma(\theta))^{-1}-\kappa \tau
$$

Now, from this fact, applying Lemma 6.2.3 to $J_{\theta}, \mathfrak{a}(\theta)$ and each number in $[0, \sigma(\theta)]$, one can easily construct an immersion $F: J_{\theta} \times[0, \varepsilon] \rightarrow V$ with the required properties.

To apply Lemma 6.2.2, moreover, we need:

Assertion 3. For any connected component $W^{\prime}$ of $f^{-1}((-\infty, v])$, there exists a point $z^{\prime}$ of $V$ and a real number $\eta^{\prime}$ such that

$$
\left|\eta^{\prime}\right|<\eta_{*}, \quad z^{\prime} \notin R, \quad \varphi_{s \eta^{\prime}}\left(z^{\prime}\right) \in R \quad \text { for } 0<s \leq 1, \quad \text { and } \quad \varphi_{\eta^{\prime}}\left(z^{\prime}\right) \in W^{\prime} \text {. }
$$

Proof. Since $\Sigma_{0}=\emptyset$, for such a set $W^{\prime}$ there exists an admissible tangential curve $\mathfrak{b}:[0, \infty) \rightarrow V$ with the image contained in $W^{\prime}$. Then since $V$ is compact, there is a limit point of $\mathfrak{b}$, i.e., a point $y$ of $V$ such that any neighborhood of $y$ in $V$ intersects the curve $\mathfrak{b}$. Using (v) of Lemma 6.2.1 and the fact $f(\mathfrak{b}(t)) \rightarrow-\infty(t \rightarrow \infty)$, we see that $y \notin R$. Therefore, since $R$ is open, taking $t$ sufficiently large, we have a point $\mathfrak{b}(t)$ such that the open ball around $\mathfrak{b}(t)$ with radius $\zeta_{*}$, where $\zeta_{*}$ is as 
in Lemma 5.1.1, intersects $V-R$. Then, we can find a point $z^{\prime}$ of $V$ $-R$ and a real number $\eta^{\prime}$ with the required properties.

We.are now ready to prove our lemma. Consider the disjoint union

$$
f^{-1}((-\infty, v])=W \cup W_{1} \cup \cdots \cup W_{m},
$$

where each $W_{i}$ is a connected component of $f^{-1}((-\infty, v])$, and $W$ is the connected component of $f^{-1}((-\infty, v])$ containing the image of $\mathfrak{a}$. Applying Assertion 3 to each $W_{i}$, we find a point $z_{i}^{\prime}$ of $V$ and a real number $\eta_{i}^{\prime}$ having the same properties as the given $z$ and $\eta$. Let $\mathfrak{b}_{i}:[0, \infty) \rightarrow V$ be an admissible tangential curve starting at $z_{i}^{\prime}$, and let $J\left(\mathfrak{G}_{i}(t)\right.$ ) be the vein through $\mathfrak{b}_{i}(t)$. The arguments in the proofs of Assertions 1 and 2 show that for each $i$, there exists a positive number $\tilde{\theta}_{i}$ satisfying the following condition: for almost every $\theta$ in $\left[\tilde{\theta}_{i}, \infty\right)$, there exists an immersion

$$
F_{i}: J\left(\mathfrak{b}_{i}(\theta)\right) \times[0, \varepsilon] \rightarrow V
$$

with the same properties (i)-(v) as in Lemma 6.2.2.

Taking a real number $w$ so that

$$
w<\min \left(f(\mathfrak{a}(\tilde{\theta})), f\left(\mathfrak{b}_{1}\left(\tilde{\theta}_{1}\right)\right), \cdots, f\left(\mathfrak{b}_{m}\left(\tilde{\theta}_{m}\right)\right)\right),
$$

and so that $w$ is a regular value of $f$ (in other words $f^{-1}(w)$ is a union of nonsingular veins), and applying Lemma 6.2.2 to $f^{-1}(w)$, which has a "nice fence", we conclude that the leaf $L(z)$ is compact, which proves Lemma 6.2.4.

Remark 6.2.3. With the same hypotheses and notation of Lemma 6.2.1, if $\mathrm{Cl}\left(\Sigma_{1} \cap R\right) \subset R$, and if a point $z$ of $V$ and a real number $\eta$ satisfy the conditions (i), (ii) of Lemma 6.2.4, then the leaf $L(z)$ through $z$ is compact.

This may be seen as follows. From Remark 6.2.1, we see that the one-form $\omega \mid R$ on $R$ can be written as

$$
\omega \mid R=P \alpha,
$$

where $P$ is a positive-valued function on $R$, and $\alpha$ is a nonsingular, closed one-form on $R$. By Lemma 2.2.1, for each leaf $N$ in $R$, we have

$$
\omega^{\prime} \mid N=d(\log P \mid N) \text {. }
$$

Using this and the compactness of $\mathrm{Cl}\left(\Sigma_{1} \cap R\right)$, we find a real number $\Delta$ 
such that for any leaf $N$ in $R$ and a first integral $g: N \rightarrow \boldsymbol{R}$ of $\omega^{\prime} \mid N$ with $g(\pi(N))=0$, where $\pi: R \rightarrow S^{1}(p)$ is as in Lemma 6.2.1, we have

$$
g^{-1}((-\infty, \Delta]) \cap \Sigma_{1}=\phi .
$$

Using this fact, we can find a point $\tilde{z}$ and a number $\tilde{\eta}$ satisfying all the conditions (i)-(iv) of Lemma 6.2.4, and such that $\tilde{z} \in L(z)$. Consequently, our assertion follows from Lemma 6.2.4.

LEMMA 6.2.5. Suppose $\Sigma_{0}=\emptyset$. If there exist two distinct compact leaves $K_{1}, K_{2}$, then the distance between $K_{1}$ and $K_{2}$ is not less than $\zeta_{*}$, where $\zeta_{*}$ is the same constant as in Lemma 5.1.1. In particular, the number of compact leaves is finite.

Proof. If there were two points $x, y$ such that $x \in K_{1}, y \in K_{2}$, dis $(x, y)$ $<\zeta_{*}$, then by Lemma 5.1.1 there would exist a real number $\eta$ such that $|\eta|<\eta_{*}, \varphi_{\eta}(x) \in K_{2}$. On the other hand, by Lemma 4.1.4, there exists an admissible tangential curve $a:[0, \infty) \rightarrow V$ with the initial point $x$. By Lemmas 4.1.2 and 4.1.3, the $\eta$-lift of $\mathfrak{a}$, which is contained in compact $K_{2}$, would approach compact $K_{1}$. This contradiction proves our lemma.

Proof of Theorem II. Let $p \in \Sigma_{n}$, and let $R$ be as in Lemma 6.2.1. By Remark 6.2.1, we know that $R$ is a $C^{r}$ fibre bundle over $S^{1}$, whose fibres are noncompact, simply connected leaves. Hence, the required imbedding is given by the inclusion map $R \subset V$. This proves (i)-(iii) of our theorem. Let $z$ be a point of $\mathrm{Cl} R-R$. In order to prove that the leaf through $z$ is compact, we consider the subset $Q$ of $\left[-\eta_{*}, \eta_{*}\right]$ consisting of those points $s$ such that $\varphi_{s}(z) \in R$, where $\eta_{*}$ is as in Lemma 4.1.3. It is clear that $Q$ is open in $\left[-\eta_{*}, \eta_{*}\right]$, and $0 \in \mathrm{Cl} Q-Q$. Hence there is an infinite sequence $q_{1}, q_{2}, \cdots, q_{i} \rightarrow 0$, of points in $Q$. Taking a subsequence if necessary, we may assume that every $q_{i}$ has the same sign, say every $q_{i}$ is positive. We shall prove that there exists $q_{i}$ such that $\left(0, q_{i}\right) \subset Q$. Then, putting $\eta=q_{i}$, and applying Remark 6.2.3, one will prove that the leaf through $z$ is compact. Now, suppose that for any $q_{i}$, the interval $\left(0, q_{i}\right)$ were not contained in $Q$. Then for each $q_{i}$, there would exist $r_{i}$ such that

$$
0<r_{i}<q_{i}, \quad\left(r_{i}, q_{i}\right) \subset Q, \quad r_{i} \notin Q .
$$

By Remark 6.2.3, the leaf through $\varphi_{r_{i}}(z)$ would be compact. Hence we should have an infinite number of compact leaves, which contradicts 
Lemma 6.2.5. Finally, for any leaf $L$ in $R$, we shall prove $\mathrm{Cl} L-L=$ Cl $R-R$. Let $z \in \mathrm{Cl} L-L$. From (v) of Lemma 6.2.1, we see that $z \notin R$. Hence $z \in \mathrm{Cl} R-R$. Conversely, let $z \in \mathrm{Cl} R-R$. The same argument as in the proof of Assertion 1, Lemma 6.2.2, shows that $z \in \mathrm{Cl} L$. Hence $z \in \mathrm{Cl} L-L$. This completes the proof of Theorem II.

\subsection{The rationality of $\boldsymbol{\omega}^{\prime} \mid \boldsymbol{K}_{i}$. We shall prove:}

Proposition 6.3.1. With the hypotheses and notation of Theorem II, for the "limit" compact leaves $K_{i}, i=1, \cdots, m$, the one-forms $\omega^{\prime} \mid K_{i}$ are rational, i.e., for any point $z$ of $K_{i}$ there exists an open set $S$ in $K_{i}$ containing $z$, and a function $P: S \rightarrow R$ such that $\omega^{\prime} \mid S=d P$, and every inverse image $P^{-1}(v), v \in \boldsymbol{R}$, is compact.

Proof. For simplicity of notation, put $K=K_{i}$. In order to make the method clear, we shall first go through the proof for the case when $\omega^{\prime} \mid K$ is nonsingular, in other words $K \cap \Sigma=\emptyset$. In that case, we have at once:

Assertion 1. There exists a tubular neighborhood $T$ of $K$ and $a$ projection $\chi: T \rightarrow K$ such that $T \cap \Sigma=\emptyset$, and such that

(i) $\chi$ is compatible with $X$, i.e., for any $x \in T, \chi_{*}\left(X_{x}\right)=0$, where $\chi_{*}: T_{x}(V) \rightarrow T_{\chi(x)}(K)$ is the induced map;

(ii) for any $x \in T$, the tangent subspace of $T_{x}(V)$ defined by $\omega_{x}=$ $\omega_{x}^{\prime}=0$, is mapped by $\chi_{*}$ into a subspace of $T_{x(x)}$ sufficiently close to the subspace defined by $\omega_{x(x)}=\omega_{x(x)}^{\prime}=0$.

On the other hand, the proof of Theorem II shows that for the $K$, $T$ above, there exists a nonsingular, compact vein $J$ contained in $T$ and an immersion $F: J \times[0, \varepsilon] \rightarrow V$ such that

(i) the restriction $F \mid J \times 0$ is the identity;

(ii) for each $s \in[0, \varepsilon]$, the image $F(J \times s)$ is contained in a leaf, and $F(J \times \varepsilon) \subset K$;

(iii) $F$ is "tangent" to $X$, more precisely, for each $x \in J$, there exists an imbedding $\rho:[0, \varepsilon] \rightarrow \boldsymbol{R}$ satisfying

$$
\rho(0)=0, F(x, s)=\varphi_{\rho(s)}(x) \quad \text { for all } s \in[0, \varepsilon] .
$$

Since $\chi$ is a projection compatible with $X$, the restriction $\chi \mid J: J \rightarrow K$ is an immersion. Furthermore, from (ii) of Assertion 1 it follows that for any $x \in J$, the subspace $\chi_{*}\left(T_{x}(J)\right)$ of $T_{x(x)}(K)$ is transversal to the vector 
$Y_{\chi(x)}$, i.e., does not contain $Y_{\chi(x)}$. Using the immersion $F$, we see that the cohomology class $\left[(\chi \mid J)^{*} \omega^{\prime}\right] \in H^{1}(J ; R)$ is trivial. Consequently, our assertion follows from Proposition 2.2.2.

For the proof in the case where $\omega^{\prime} \mid K$ has singular points, we introduce the following word: A tubular neighborhood $T$ of the compact leaf $K$, with a projection $\chi: T \rightarrow K$, will be said to be "nice" if

(i) $\chi$ is compatible with $X$;

(ii) for any $x \in T-(T \cap \Sigma)$, the subspace of $T_{x}(V)$ defined by $\omega_{x}$ $=\omega_{x}^{\prime}=0$, is mapped by $\chi_{*}$ into a subspace of $T_{x(x)}(K)$ which is transversal to the vector $Y_{\chi(x)}$.

It is obvious that if such $T, \chi$ exist, our proposition follows from Proposition 2.2.2 again, because $K \cap \Sigma_{1}=\emptyset$. In the general case, we use the following technique. First, take a $C^{r+1}$ positive-valued function $h$ sufficiently close to the constant function 1 so that the critical cycle $\tilde{\Sigma}$ for the completely integrable one-form $\tilde{\omega}=h \omega$, is transversal to $K$. Using the fact that every point of $\Sigma$ has an $\mathscr{F}$-chart of "type $(\lambda)$ " or of "type $(*, \mu)$ " (see Proposition 4.1.1), one has such a "deformation" of $\omega$. It should be also noted that $\tilde{\omega}$ defines the same leaves as $\omega$, i.e., any leaf of $(V, \tilde{\omega})$ is a leaf of $(V, \omega)$, and vice versa. Next, change the vector field $X$ to a $C^{r}$ vector field $\tilde{X}$ with $\tilde{\omega}(\tilde{X})=1$ so that for any $p \in K \cap \tilde{\Sigma}$ and a suitable neighborhood $U$ of $p$ in $V, \tilde{X}$ is expressed on $U$ as

$$
\tilde{X} \mid U=a(x)\left(\partial / \partial x^{n+1}\right),
$$

where $a(x)$ is a function on $U$, and $\left(U, f ; x^{1}, \cdots, x^{n+1}\right)$ is an $\mathscr{F}$-chart of type $(\lambda)(\lambda=0,1, \cdots, n)$, for $(V, \tilde{\omega})$. Noting that since $\tilde{\Sigma}$ is transversal to $K$, any point $p$ of $K \cap \tilde{\Sigma}$ is not of type (*), and using again the fact that for $\lambda=0, \cdots, n$, every point of type $(\lambda)$ has an $\mathscr{F}$-chart of type $(\lambda)$ for $(V, \tilde{\omega})$, one obtain such a vector field $\tilde{X}$. Now, from the properties of $\tilde{X}$, it follows that $K$ has a "nice" tubular neighborhood with respect to $\tilde{\omega}, \tilde{X}$, and hence that $\tilde{\omega}^{\prime} \mid K$ is rational, where $\tilde{\omega}^{\prime}=-\mathscr{L}_{\tilde{X}} \tilde{\omega}$. Using the relation

$$
\left(\tilde{\omega}^{\prime}-\omega^{\prime}\right) \mid K=d(\log h \mid K),
$$

we conclude that $\omega^{\prime} \mid K$ is also rational. This completes the proof of Proposition 6.3.1.

6.4. Proof of Lemma 6.1.1. With the notation in 6.1 , we have the 
following lemma, which implies Lemma 6.1.1.

LEMMA 6.4.1. (i) There exist two positive constants $c_{1}, d_{1}$ such that the inequality

$$
c_{1} \cdot \operatorname{diam}_{J}(J) \leq \operatorname{mes}_{n-1}(J)
$$

holds for any nonsingular, compact vein $J$ satisfying

$$
\operatorname{diam}_{J}(J)>d_{1} \text {. }
$$

(ii) If $\Sigma_{0}=\emptyset$, and $\Sigma_{n-1}=\emptyset$, then there exist three positive constants $c_{2}, d_{1}$,e such that the inequality

$$
c_{2} \cdot \mu \cdot \operatorname{mes}_{n-1}(J) \leq \operatorname{mes}_{n}\left(\bigcup_{0 \leq u \leq \mu} J[u]\right)
$$

holds for $0<\mu \leq e$ and for any nonsingular, compact vein $J$ satisfying the following conditions:

$$
\begin{aligned}
& \operatorname{diam}_{J}(J)>d_{1} ; \\
& J \cap J[u]=\emptyset \quad \text { for } 0<u \leq \mu .
\end{aligned}
$$

(iii) There exists a positive constant $c_{3}$ such that the inequality

$$
c_{3} \cdot \sigma \cdot \operatorname{mes}_{n}(H) \leq \operatorname{mes}_{n+1}\left(\bigcup_{0 \leq s \leq \sigma} \varphi_{s}(H)\right)
$$

holds for $0<\sigma \leq 1$ and for any compact $n$-submanifold $H$ of $V$, with or without boundary, satisfying the following conditions: $H$ is contained in a leaf; and

$$
H \cap \varphi_{s}(H)=\emptyset \quad \text { for } 0<s \leq \sigma .
$$

We shall first prove the part (iii).

Proof of (iii). It is obvious that there is a positive constant $a$ such that

$$
a \cdot \operatorname{mes}_{n}(D) \leq \operatorname{mes}_{n}\left(\varphi_{s}(D)\right)
$$

for $0 \leq s \leq 1$ and any imbedded closed $n$-disk $D$ in $V$. Then, put

$$
b=\min \left\|X_{\varphi_{s}(x)}-\left(X_{\varphi_{s}(x)} \mid\left(\varphi_{s}\right)_{*} P_{x}\right)\right\|
$$

the minimum being taken over all $x$ in $V$ and all $s$ in $[0,1]$, where $P_{x}$ is the subspace $\omega_{x}^{-1}(0)$ of $T_{x}(V)$, and $X_{\varphi_{s}(x)} \mid\left(\varphi_{s}\right)_{*} P_{x}$ is the orthogonal 
component of the vector $X_{\varphi_{s}(x)}$ to the subspace $\left(\varphi_{s}\right)_{*} P_{x}$. Then it is easily verified that $c_{3}=a b$ is the required constant. The part (iii) is proved.

Proof of (i). Let $T^{*}, \tilde{T}^{*}$ be two $Y$-preferred neighborhoods of $\Sigma$ such that $\mathrm{Cl} \tilde{T}^{*} \subset T^{*}$ (see 4.2 for the definition and the existence). Let $b_{1}^{*}$, $\tilde{b}_{1}^{*}, \tilde{b}_{2}^{*}$ be the same constants as in (v) of Definition 4.2 .1 , i.e., satisfy the following conditions:

(1) $\operatorname{diam}_{J^{*}}\left(J^{*}\right)<b_{1}^{*} \quad$ for any $T^{*}$-vein $J^{*}$;

(2) $\operatorname{diam}_{\tilde{J}^{*}}\left(\tilde{J}^{*}\right)<\tilde{b}_{1}^{*} \quad$ for any $\tilde{T}^{*}$-vein $\tilde{J}^{*}$;

(3) $\operatorname{mes}_{n-1}\left(\tilde{J}^{*}\right)>\tilde{b}_{2}^{*} \quad$ for any noncompact $\tilde{T}^{*}$-vein $\tilde{J}^{*}$.

Let $W_{1}, \cdots, W_{m}$ be open sets of $V$ such that

$$
V=\tilde{T}^{*} \cup \underset{1 \leq i \leq m}{\bigcup} W_{i}, \quad \Sigma \cap \mathrm{Cl}\left(\bigcup_{1 \leq i \leq m} W_{i}\right)=\emptyset .
$$

Let $\delta_{1}$ be a Lebesgue number of the open covering $\left\{\tilde{T}^{*}, W_{1}, \cdots, W_{m}\right\}$. Put

$$
\delta=\min \left(\delta_{1}, \delta_{2} / 2\right)
$$

where $\delta_{2}$ is the distance between the sets $\tilde{T}^{*}$ and $V-T^{*}$. Then, since $\Sigma \cap \mathrm{Cl}\left(\cup_{1 \leq i \leq m} W_{i}\right)=\emptyset$, there is a positive constant $\Delta$ such that

$$
\operatorname{mes}_{n-1}\left(B_{J}(x, \delta)\right)>\Delta
$$

for any $x \in \bigcup_{1 \leq i \leq m} W_{i}$ satisfying $B_{J}(x, \delta) \subset \bigcup_{1 \leq i \leq m} W_{i}$, where $B_{J}(x, \delta)$ denotes an open ball in a vein $J$ through $x$, with center $x$ and radius $\delta$. We shall prove that the constants defined by

$$
c_{1}=\min \left(\Delta / 2 \delta, \tilde{b}_{2}^{*} / b_{1}^{*}\right), \quad d_{1}=\tilde{b}_{1}^{*},
$$

have the required properties.

Now, let a nonsingular, compact vein $J$ with $\operatorname{diam}_{J}(J)>d_{1}$ be given. Put $\lambda=\operatorname{diam}_{J}(J)$. Since $J$ is a closed manifold, there is a curve $\gamma:[0, \lambda]$ $\rightarrow J$ which is a geodesic in $J$ and is parametrized by arc-length, and is minimal in the following sense:

$$
\operatorname{dis}_{J}\left(\gamma(t), \gamma\left(t^{\prime}\right)\right)=\left|t-t^{\prime}\right| \quad \text { for any } t, t^{\prime} \in[0, \lambda] .
$$

Let $s$ be an integer satisfying

$$
\lambda / 2 \delta \leq s<(\lambda / 2 \delta)+1,
$$

and put $t_{0}=0, t_{1}=2 \delta, \cdots, t_{s-1}=2(s-1) \delta$. Denote by $E$ the set of all elements $i \in\{0, \cdots, s-1\}$ such that $B_{J}\left(\gamma\left(t_{i}\right), \delta\right) \cap \tilde{T}^{*} \neq \emptyset$, where $B_{J}\left(\gamma\left(t_{i}\right), \delta\right)$ 
is the open ball in $J$, with center $\gamma\left(t_{i}\right)$ and radius $\delta$. Put $E^{c}=$

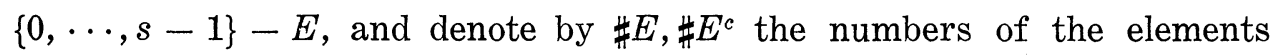
of the sets $E, E^{c}$, respectively. Then

Assertion 1. (a) If $i \in E$, then we have

$$
B_{J}\left(\gamma\left(t_{i}\right), \delta\right) \cap J \cap \tilde{T}^{*} \neq \emptyset, \quad B_{J}\left(\gamma\left(t_{i}\right), \delta\right) \subset J \cap T^{*} .
$$

(b) If $i \in E^{c}$, then we have

$$
B_{J}\left(\gamma\left(t_{i}\right), \delta\right) \cap J \cap \tilde{T}^{*}=\emptyset, \quad \operatorname{mes}_{n-1}\left(B_{J}\left(\gamma\left(t_{i}\right), \delta\right)\right)>\Delta .
$$

Proof of (a). The former part is obvious. The latter part follows from the facts that $\operatorname{dis}_{V}\left(\tilde{T}^{*}, V-T^{*}\right) \geq 2 \delta \operatorname{dis}_{J}(x, y) \geq \operatorname{dis}_{V}(x, y)$ for $x, y \in J$. Proof of (b). The former part is obvious. Since $\delta$ is a Lebesgue number of the open covering $\left\{\tilde{T}^{*}, W_{1}, \cdots, W_{m}\right\}$, we have $B_{J}\left(\gamma\left(t_{i}\right), \delta\right) \subset$ $\cup W_{j}$ for $i \in E^{c}$. Hence the latter part follows from the definition of $\Delta$.

On the other hand,

Assertion 2. We have a disjoint union

$$
J \cap \tilde{T}^{*}=\tilde{J}_{1}^{*} \cup \cdots \cup \tilde{J}_{k}^{*},
$$

where each $\tilde{J}_{j}^{*}$ is a noncompact $\tilde{T}^{*}$-vein with $\operatorname{mes}_{n-1}\left(\tilde{J}_{j}^{*}\right)>\tilde{b}_{2}^{*}$.

Proof. We shall prove that a connected component $\tilde{J}^{*}$ of $J \cap \tilde{T}^{*}$, which is by definition a $\tilde{T}^{*}$-vein, is noncompact. Then (3) above will imply $\operatorname{mes}_{n-1}\left(\tilde{J}^{*}\right)>\tilde{b}_{2}^{*}$. Now, suppose that $\tilde{J}^{*}$ were compact. Then $\tilde{J}^{*}$ would coincide with $J$. Since $\operatorname{diam}_{\tilde{J}^{*}}\left(\tilde{J}^{*}\right)<\tilde{b}_{1}^{*}$ by (2), we should have $\operatorname{diam}_{J}(J)<\tilde{b}_{1}^{*}=d_{1}$, which is a contradiction. Assertion 2 is proved.

Now, for each $\tilde{J}_{j}^{*}, j=1, \cdots, k$, since $\tilde{T}^{*} \subset T^{*}$, there is a $T^{*}$-vein $J_{j}^{*}$ such that $\tilde{J}_{j}^{*} \subset J_{j}^{*}$. Then, for each $J_{j}^{*}$, since $\gamma$ is minimal, and $\operatorname{diam}_{J_{j}^{*}}\left(J_{j}^{*}\right)<b_{1}^{*}$, we observe that the number of the elements $i \in E$ satisfying $B_{J}\left(\gamma\left(t_{i}\right), \delta\right) \subset J_{j}^{*}$, is bounded by $b_{1}^{*} / 2 \delta$. From Assertion 1 (a), we know that if $i \in E$, then $B_{J}\left(\gamma\left(t_{i}\right), \delta\right)$ is contained in some $J_{j}^{*}, j=1, \cdots, k$. Consequently, we obtain

$$
\# E \leq k b_{1}^{*} / 2 \delta \text {. }
$$

Since $J$ contains a disjoint union

$$
\bigcup_{i \in E^{c}} B_{J}\left(\gamma\left(t_{i}\right), \delta\right) \cup \underset{1 \leq j \leq k}{ } \tilde{J}_{j}^{*},
$$


by Assertions 1 (b) and 2, we have

$$
\operatorname{mes}_{n-1}(J)>\left(\# E^{c}\right) \Delta+k \tilde{b}_{2}^{*} .
$$

Since $\sharp E \cdot 2 \delta / b_{1}^{*} \leq k$, it follows that

$$
\begin{aligned}
\operatorname{mes}_{n-1}(J) & >\left(\# E^{c}\right) \Delta+\# E \cdot\left(2 \delta / b_{1}^{*}\right) \tilde{b}_{2}^{*}, \quad \text { and hence } \\
& >\left(\# E^{c}+\# E\right) \cdot \min \left(\Delta,\left(2 \delta / b_{1}^{*}\right) \tilde{b}_{2}^{*}\right) .
\end{aligned}
$$

Since $s=\sharp\left(E \cup E^{c}\right)$, and $s \geq \lambda / 2 \delta$, we obtain finally

$$
\operatorname{mes}_{n-1}(J)>\lambda \cdot \min \left(\Delta / 2 \delta, \tilde{b}_{2}^{*} / b_{1}^{*}\right)=c_{1} \cdot \operatorname{diam}_{J}(J),
$$

which proves the part (i) of Lemma 6.4.1.

Proof of (ii). Let $T^{*}$ be a $Y$-preferred neighborhood of $\Sigma$, as before. Let $S$ be an open set of $V$ such that

$$
\Sigma \subset S \subset \mathrm{Cl} S \subset T^{*} \text {. }
$$

Put

$$
\delta=\operatorname{dis}_{V}\left(S, V-T^{*}\right), \quad a=\min _{x \in V-S}\left\|Y_{x}\right\|, \quad e=a \delta .
$$

It is easily verified that there is a positive constant $c_{2}^{\prime}$ such that the inequality

$$
c_{2}^{\prime} \cdot \mu \cdot \operatorname{mes}_{n-1}(G)<\operatorname{mes}_{n}\left(\bigcup_{0 \leq u \leq \mu} G[u]\right)
$$

holds for $0<\mu \leq e$ and any compact or bounded open $(n-1)$-manifold $G$ which is contained in a vein and satisfies the conditions

$$
S \cap \bigcup_{0 \leq u \leq \mu} G[u]=\emptyset, \quad G \cap G[u]=\emptyset \quad \text { for } 0<u \leq \mu .
$$

Now, for this constant $c_{2}^{\prime}$ and the same constants $b_{1}^{*}, b_{2}^{*}, b_{3}^{*}, d_{1}^{*}$ as in (v), (viii) of Definition 4.2.1, put

$$
c_{2}=\min \left(c_{2}^{\prime}, d_{1}^{*} b_{2}^{*} / b_{3}^{*}\right), \quad d_{1}=b_{1}^{*} .
$$

We shall prove that these $c_{2}, d_{1}$, and $e$ above have the desired properties.

Assertion 1. If a $T^{*}$-vein $J^{*}$ satisfies

$$
S \cap \bigcup_{0 \leq u \leq e} J^{*}[u] \neq \emptyset,
$$

then 


$$
\bigcup_{0 \leq u \leq e} J^{*}[u] \subset T^{*}
$$

To prove this, for an admissible tangential curve $a:[0, \tau] \rightarrow V$, note that if $\operatorname{Im} \mathfrak{a} \cap S \neq \emptyset$, and $\operatorname{Im} \mathfrak{a} \cap\left(V-T^{*}\right) \neq \emptyset$, then $\tau \geq \delta$, and that if $\operatorname{Im} \mathfrak{a} \cap S=\emptyset$, then

$$
\int_{a[0, \tau]} \omega^{\prime}=-\int_{0}^{\tau}\left\|Y_{a(t)}\right\| d t \leq-a \tau .
$$

Then we observe that if $a:[0, \tau] \rightarrow V$ is an admissible tangential curve such that

$$
\operatorname{Im} a \cap S \neq \emptyset, \quad \int_{a[0, \tau]} \omega^{\prime} \geq-a \delta=-e,
$$

then $\operatorname{Im} a \subset T^{*}$. This proves Assertion 1.

Assertion 2. If a noncompact $T^{*}$-vein $J^{*}$ satisfies

$$
\bigcup_{0 \leq u \leq e} J^{*}[u] \subset T^{*},
$$

then

$$
\operatorname{mes}_{n}\left(\bigcup_{0 \leq u \leq \mu} J^{*}[u]\right)>c_{2} \cdot \mu \cdot \operatorname{mes}_{n-1}\left(J^{*}\right) \quad \text { for } 0<\mu \leq e .
$$

Proof. Let $L$ be the leaf containing the set $\cup_{0 \leq u \leq e} J^{*}[u]$, and let $L^{*}$ be the connected component of the subset $L \cap T^{*}$ of $L$, containing $\bigcup_{0 \leq u \leq e} J^{*}[u]$. Let $Q$ be an imbedded closed $n$-disk in $L$ containing $L^{*}$, and let $h: Q \rightarrow \boldsymbol{R}$ be a function satisfying $\omega^{\prime} \mid Q=d h$. For $0<\mu \leq e$, let $a:[0, \tau] \rightarrow V$ be an admissible tangential curve such that $\mathfrak{a}(0) \in J^{*}$, $\mathfrak{a}(\tau) \in J^{*}[\mu]$. Then it is clear that $\operatorname{Im} \mathfrak{a} \subset T^{*}$. Put $h_{t}=h(\mathfrak{a}(t))$. It is clear that $\operatorname{Im} \mathfrak{a} \subset h^{-1}\left(\left[h_{\tau}, h_{0}\right]\right)$, and $\mu=h_{0}-h_{\tau}$. We denote by $\left(\left(h^{-1}\left[h_{\tau}, h_{0}\right]\right.\right.$ $\left.\cap T^{*}\right)$ ) the connected component of the subset $h^{-1}\left(\left[h_{\tau}, h_{0}\right]\right) \cap T^{*}$ of $Q$, containing Ima. Using the assumption $\Sigma_{n-1}=\emptyset$ and the property (vii) of $T^{*}$, one can prove

$$
\bigcup_{0 \leq u \leq \mu} J^{*}[u]=\left(\left(h^{-1}\left(\left[h_{\tau}, h_{0}\right]\right) \cap T^{*}\right)\right) .
$$

Then, by the property (viii) of $T^{*}$, we obtain

$$
\operatorname{mes}_{n}\left(\bigcup_{0 \leq u \leq \mu} J^{*}[u]\right) \geq d_{1}^{*} \cdot \mu \cdot \inf _{t \in[0, \tau]} \operatorname{mes}_{n-1}\left(\left(h^{-1}\left(h_{t}\right) \cap T^{*}\right)\right),
$$

where $\left(\left(h^{-1}\left(h_{t}\right) \cap T^{*}\right)\right)$ denotes the connected component of the subset 
$h^{-1}\left(h_{t}\right) \cap T^{*}$ of $Q$, containing the point $\mathfrak{a}(t)$. It is clear that $\left(\left(h^{-1}\left(h_{t}\right) \cap\right.\right.$ $\left.T^{*}\right)$ ) is a $T^{*}$-vein. Furthermore, using the property (vi) of $T^{*}$ and the assumptions that $\Sigma_{0}=\emptyset$, and $J^{*}$ is noncompact, we know that this $T^{*}$ vein is also noncompact. Hence by the property (v) of $T^{*}$, we have

$$
\inf _{t \in[0, \tau]} \operatorname{mes}_{n-1}\left(\left(h^{-1}\left(h_{t}\right) \cap T^{*}\right)\right) \geq b_{2}^{*}>\left(b_{2}^{*} / b_{3}^{*}\right) \cdot \operatorname{mes}_{n-1}\left(J^{*}\right) .
$$

From this and the inequality above, we obtain the required inequality. Assertion 2 is proved.

Now, let $\mu$ be a positive number $\leq e$. Let a nonsingular, compact vein $J$ such that

$$
\operatorname{diam}_{J}(J)>b_{1}^{*}, \quad J \cap J[u]=\emptyset \quad \text { for } 0<u \leq \mu,
$$

be given. Then using Assertion 2 in the proof of the part (i) and the property (ix) of $T^{*}$, we have a disjoint union

$$
J=G \cup J_{1}^{*} \cup \cdots \cup J_{k}^{*},
$$

where $G$ is a compact $(n-1)$-submanifold of $J$ such that $G \cap T^{*}=\emptyset$, and each $J_{j}^{*}$ is a noncompact $T^{*}$-vein. It is clear that

$$
G \cap G[u]=\emptyset, \quad J_{j}^{*} \cap J_{j}^{*}[u]=\emptyset \quad \text { for } 0<u \leq \mu, j=1, \cdots, k .
$$

Furthermore, by Assertion 1, we know that $S \cap \cup_{0 \leq u \leq \mu} G[u]=\emptyset$, and that if $S \cap \bigcup_{0 \leq u \leq \mu} J_{j}^{*}[u] \neq \emptyset$, then $\bigcup_{0 \leq u \leq \mu} J_{j}^{*}[u] \subset T^{*}$. Hence we have

$$
\begin{gathered}
\operatorname{mes}_{n}\left(\bigcup_{0 \leq u \leq \mu} G[u]\right)>c_{2} \cdot \mu \cdot \operatorname{mes}_{n-1}(G), \\
\operatorname{mes}_{n}\left(\bigcup_{0 \leq u \leq \mu} J_{j}^{*}[u]\right)>c_{2} \cdot \mu \cdot \operatorname{mes}_{n-1}\left(J_{j}^{*}\right) \quad \text { for } j=1, \cdots, k .
\end{gathered}
$$

Using the assumption $\Sigma_{n-1}=\emptyset$ and the property (vii) of $T^{*}$, one can prove that

$$
\bigcup_{0 \leq u \leq \mu} J[u]=\bigcup_{0 \leq u \leq \mu} G[u] \cup \bigcup_{1 \leq j \leq k} \bigcup_{0 \leq u \leq \mu} J_{j}^{*}[u]
$$

is a disjoint union. Consequently, we obtain

$$
\begin{aligned}
\operatorname{mes}_{n}\left(\bigcup_{0 \leq u \leq \mu} J[u]\right) & \geq c_{2} \cdot \mu\left(\operatorname{mes}_{n-1}(G)+\sum_{0 \leq j \leq k} \operatorname{mes}_{n-1}\left(J_{j}^{*}\right)\right) \\
& =c_{2} \cdot \mu \cdot \operatorname{mes}_{n-1}(J) .
\end{aligned}
$$

This proves the part (ii) and completes the proof of Lemma 6.4.1. 


\section{§ 7. Proof of Theorem III}

We fix an $\omega$-preferred Riemannian structure and use the notations $\left\{\varphi_{s}\right\}, \omega^{\prime}$ as in $\S 5$.

7.1. Proof of Theorem III. We begin by recalling a definition and a lemma by Reeb. A leaf $L$ is said to be locally dense if for some point $x$ of $L$, the closure of the intersection of the set $\left\{\varphi_{s}(x)\right\}_{|s| \leq 1}$ and $L$ contains a neighborhood of $x$ in $\left\{\varphi_{s}(x)\right\}_{|s| \leq 1}$. It is clear that if a leaf $L$ is locally dense, then for any point of $L$, the same property as above is satisfied.

LEMMA 7.1.1. If the closure of a leaf $M$ contains a locally dense leaf, then $M$ itself is locally dense.

This is proved in [6, p. 108, (A, II, 10)].

For the proof of our theorem, we need two lemmas.

LEMMA 7.1.2. Suppose that $\Sigma_{n}=\emptyset$. Then for any nonempty open subset $U$ of $V$, there exists a point $x$ of $U$ such that the leaf through $x$ is locally dense.

Proof. Suppose that this were not the case, and let $U$ be a nonempty open subset of $V$ such that for any point $x$ of $U$, the leaf through $x$ is not locally dense. Fix a point $x_{0}$ of $U$, and let $h$ be a positive number such that the set $\left\{\varphi_{s}\left(x_{0}\right)\right\}_{|s| \leq h}$ is imbedded in $U$. For simplicity of notation, we identify the interval $[-h, h]$ with the transversal segment $\left\{\varphi_{s}\left(x_{0}\right)\right\}_{|s| \leq n}$. We also denote by $L(y)$ a leaf through a point $y$ of $V$. Now, by assumption, for the leaf $L\left(x_{0}\right)$, the closed subset

$$
\mathrm{Cl}\left(L\left(x_{0}\right) \cap[-h, h]\right) \subset[-h, h]
$$

does not contain any neighborhood of $x_{0}$ in $[-h, h]$. Hence there exists an open interval $\left(a_{1}, b_{1}\right) \neq \emptyset$ such that

$$
\left(a_{1}, b_{1}\right) \subset[-h, h], \quad\left(a_{1}, b_{1}\right) \cap \mathrm{Cl}\left(L\left(x_{0}\right) \cap[-h, h]\right)=\emptyset .
$$

By our assumption again, for a point $x_{1}$ of $\left(a_{1}, b_{1}\right)$ and a leaf $L\left(x_{1}\right)$, the closed subset

$$
\mathrm{Cl}\left(L\left(x_{1}\right) \cap[-h, h]\right) \subset[-h, h]
$$

does not contain any neighborhood of $x_{1}$ in $\left[a_{1}, b_{1}\right]$, and hence there exists an open interval $\left(a_{2}, b_{2}\right) \neq \emptyset$ such that 


$$
\left(a_{2}, b_{2}\right) \subset\left[a_{1}, b_{1}\right], \quad\left(a_{2}, b_{2}\right) \cap \mathrm{Cl}\left(L\left(x_{1}\right) \cap[-h, h]\right)=\emptyset .
$$

Repeating this process, we obtain an infinite sequence of points $x_{0}, x_{1}$, $x_{2}, \cdots$ in $[-h, h]$ such that if $i<j$, then

$$
x_{j} \notin \mathrm{Cl}\left(L\left(x_{i}\right) \cap[-h, h]\right) .
$$

For these points $x_{i}$, we define points $x_{i}^{\infty}$ of $V$ as follows. Since $\Sigma_{n}=\emptyset$, by Lemma 4.1.4, there exists, for each $x_{i}$, an admissible tangential curve $\mathfrak{a}_{i}:(-\infty, 0] \rightarrow V$ with $\mathfrak{a}_{i}(0)=x_{i}$. Since $V$ is compact, an infinite sequence $\mathfrak{a}_{i}(0), \mathfrak{a}_{i}(-1), \mathfrak{a}_{i}(-2), \cdots$ of points of $V$ has a convergent subsequence, whose limit point we denote by $x_{i}^{\infty}$. Thus, we have an infinite sequence $x_{0}^{\infty}, x_{1}^{\infty}, x_{2}^{\infty}, \ldots$ in $V$. Let $\zeta_{*}$ be the same constant as in Lemma 5.1.1. Then there exist two positive integers $i, j$ with $i<j$ such that the distance between $x_{i}^{\infty}$ and $x_{j}^{\infty}$ is smaller than $\zeta_{*} / 3$. For these $i, j$, there exists a positive number $\delta$ such that

$$
\bigcup_{|s| \leq \delta} \varphi_{s}\left(x_{j}\right) \cap \mathrm{Cl}\left(L\left(x_{i}\right) \cap[-h, h]\right)=\emptyset .
$$

For this $\delta$ and the same constants $\alpha_{*}, \tau_{*}, \eta_{*}, \beta_{*}$ as in Lemmas 4.1.2 and 4.1.3, take a real number $\tau$ so that

$$
\tau_{*} \leq \tau, \quad \eta_{*} \exp \left(-\alpha_{*} \tau\right) /\left(1-\eta_{*} \beta_{*}\right)<\delta .
$$

For this $\tau$, by the definition of $x_{j}^{\infty}$, there exists an integer $m$ such that

$$
\tau<m, \quad \operatorname{dis}\left(a_{j}(-m), x_{j}^{\infty}\right)<\zeta_{*} / 3 .
$$

Let $k$ be a positive integer such that

$$
\operatorname{dis}\left(a_{i}(-k), x_{i}^{\infty}\right)<\zeta_{*} / 3 .
$$

Put $y=\mathfrak{a}_{i}(-k), z=\mathfrak{a}_{j}(-m)$. Then we observe that

$$
\operatorname{dis}(y, z) \leq \operatorname{dis}\left(y, x_{i}^{\infty}\right)+\operatorname{dis}\left(x_{i}^{\infty}, x_{j}^{\infty}\right)+\operatorname{dis}\left(x_{j}^{\infty}, z\right)<\zeta_{*} .
$$

Hence by Lemma 5.1.1, there is a real number $\eta$ with $|\eta|<\eta_{*}$ such that $\varphi_{\eta}(z) \in L(y)$. Let $\mathfrak{b}:[0, m] \rightarrow V$ be an admissible tangential curve defined by

$$
\mathfrak{b}(t)=\mathfrak{a}_{j}(t-m)
$$

Then $\mathfrak{b}(0)=z, \mathfrak{b}(m)=x_{j}$. By Lemma 4.1.3, there exists the $\eta$-lift $\mathfrak{c}$ of $\mathfrak{b}$ with height parameter $\sigma$ satisfying 


$$
|\sigma(t)| \leq\left(|\eta| \exp \int_{6[0, t]} \omega^{\prime}\right) /\left(1-|\eta| \beta_{*}\right), \quad t \in[0, m] .
$$

From this inequality and Lemma 4.1.2, it follows that

$$
|\sigma(m)| \leq|\eta| \exp \left(-\alpha_{*} m\right) /\left(1-|\eta| \beta_{*}\right) .
$$

Hence we have

$$
|\sigma(m)|<\eta_{*} \exp \left(-\alpha_{*} m\right) /\left(1-\eta_{*} \beta_{*}\right)<\delta .
$$

On the other hand, since the curve $c$ is contained in $L(y)$, and $L(y)$ is nothing other than $L\left(x_{i}\right)$, we conclude that $c(m)=\varphi_{\sigma(m)}\left(x_{j}\right) \in L\left(x_{i}\right)$. This and the inequality obtained above contradict the choice of $\delta$. This proves Lemma 7.1.2.

LEMMA 7.1.3. Suppose that $\Sigma_{n}=\emptyset$. Let $L$ be a locally dense leaf. Then there exists an open subset $W$ of $V$ containing $L$ such that for any point $y$ of $W$, the closure of a leaf through $y$ contains $L$.

Proof. Fix a point $x$ of $L$. Let $a:(-\infty, 0] \rightarrow V$ be an admissible tangential curve with $\mathfrak{a}(0)=x$, and consider a point $x^{\infty}$ as before, i.e., define $x^{\infty}$ to be a point of $V$ such that any neighborhood of $x^{\infty}$ contains infinitely many points of the set $\{a(-i)\}_{i=0,1, \ldots}$. Let $\zeta_{*}$ be as in Lemma 5.1.1. Then there exists a positive integer $i$ such that

$$
\operatorname{dis}\left(\mathfrak{a}(-i), x^{\infty}\right)<\zeta_{*} / 3 .
$$

We shall prove that for an open ball $B$ around the point $\mathfrak{a}(-i)$ with radius $\zeta_{*} / 3$, the closure of any leaf through points of $B$ contains the point $x$. Then one will see that the open set $W$ consisting of those leaves which intersect $B$, has the desired property (cf. [6, p. 106, (A, II, 4), $(\mathrm{A}, \mathrm{II}, 6)]$ ). Now, let $y$ be a point of $B$, and let $\varepsilon$ be a positive number. Then, first, we take a real number $\tau$ so that

$$
\tau_{*} \leq \tau, \quad \eta_{*} \exp \left(-\alpha_{*} \tau\right) /\left(1-\eta_{*} \beta_{*}\right)<\varepsilon,
$$

where $\alpha_{*}, \tau_{*}, \eta_{*}, \beta_{*}$ be as in Lemmas 4.1.2 and 4.1.3. Next, we take an integer $m$ so that

$$
\tau<m, \quad \operatorname{dis}\left(\mathfrak{a}(-m), x^{\infty}\right)<\zeta_{*} / 3 .
$$

Put $z=\mathfrak{a}(-m)$. Then we observe that

$$
\operatorname{dis}(y, z) \leq \operatorname{dis}(y, \mathfrak{a}(-i))+\operatorname{dis}\left(\mathfrak{a}(-i), x^{\infty}\right)+\operatorname{dis}\left(x^{\infty}, \mathfrak{a}(-m)\right)<\zeta_{*} .
$$


As in the proof of Lemma 7.1.2, considering an admissible tangential curve $\mathfrak{b}:[0, m] \rightarrow V$ defined by

$$
\mathfrak{b}(t)=\mathfrak{a}(t-m),
$$

we conclude that the leaf $M$ through the point $y$ intersects the transversal segment $\left\{\varphi_{s}(x)\right\}_{|s| \leq \epsilon^{*}}$. This proves that the closure of $M$ contains $x$, and proves Lemma 7.1.3.

Proof of Theorem III. By Lemma 7.1.2, the set $V_{0}$ consisting of locally dense leaves is a dense subset of $V$, which is open by Lemmas 7.1.1 and 7.1.3. This proves our theorem.

\section{§ 8. Proofs of Propositions 4.1.1. and 4.2.1.}

Propositions 4.1.1 and 4.2.1 will be proved in 8.2 and 8.3 respectively.

8.1. Preliminary lemmas. Here, we shall use the following notation. Let $0^{n-1}, 0^{n}$ and $0^{n+1}$ denote the origins of $\boldsymbol{R}^{n-1}, \boldsymbol{R}^{n}$ and $\boldsymbol{R}^{n+1}$, respectively. The sets $0^{n-1} \times \boldsymbol{R}^{2}, 0^{n} \times \boldsymbol{R}$ are to be understood as the subsets of $\boldsymbol{R}^{n+1}$. Let $\left(x^{1}, \cdots, x^{n+1}\right)$ be the standard coordinate system of $\boldsymbol{R}^{n+1}$. Denote by $x$ the point with coordinates $\left(x^{1}, \cdots, x^{n+1}\right)$. Let $U$ be a bounded, open subset of $\boldsymbol{R}^{n+1}$ containing $0^{n+1}$. Let $f$ be a positive-valued function defined on $U$, and put $F=\log f$. For a $C^{3}$ function $g$ defined on a subset of $\boldsymbol{R}^{n+1}$, we introduce the following notations:

$$
\begin{aligned}
& \partial_{i} g=\partial g / \partial x^{i}, \quad i=1, \cdots, n+1, \\
& \|\partial g(x)\|=\left(\partial_{i} g ; i \rightarrow 1, \cdots, n\right) \quad \text { (n-tuple), } \\
& \left\|\partial^{2} g(x)\right\|=\left(g_{i j}(x) ; i, j=1, \cdots, n\right) \quad(n \times n \text {-matrix }) \text {, } \\
& \left\|\partial^{3} g(x)\right\|=\left(g_{i j}(x), \frac{\partial}{\partial x^{i}} \operatorname{det}\left\|\partial^{2} g(x)\right\| ; \begin{array}{l}
i \underset{1}{\downarrow} 1, \cdots, n+1 \\
j \rightarrow 1, \cdots, n
\end{array}\right) \\
& (n+1 \times n+1 \text {-matrix }) \text {, }
\end{aligned}
$$

where $g_{i j}=\partial^{2} g / \partial x^{i} \partial x^{j}$.

The index of a real symmetric matrix is defined to be the number of negative eigenvalues. For numbers $a_{1}, \cdots, a_{m}$, we denote by diag $\left[a_{1}, \cdots\right.$, $a_{m}$ ] an $m \times m$-diagonal matrix with the diagonal elements $a_{1}, \cdots, a_{m}$. Let $\lambda, \mu$ be integers such that $0 \leq \lambda \leq n, 0 \leq \mu \leq n-1$. For a positive integer $s$ and an open subset or, more generally, an $(n+1)$-submanifold $W$ of $\boldsymbol{R}^{n+1}$, we denote by $\Gamma^{s}(W)$ the set of all $C^{s}$ diffeomorphisms 
$\Phi: W \rightarrow \Phi(W) \subset U$ which are written in the form

$$
\Phi\left(x^{1}, \cdots, x^{n+1}\right)=\left(\Phi^{1}(x), \cdots, \Phi^{n}(x), x^{n+1}\right), \quad x \in W,
$$

with suitable real-valued functions $\Phi^{i}$ defined on $W$. For a subset $S$ of $\boldsymbol{R}^{n}$ and two real numbers $a, b$ with $a<b$, we denote by $S_{a}^{b}$ the set

$$
S_{a}^{b}=\left\{\left(x^{1}, \cdots, x^{n+1}\right) \in \boldsymbol{R}^{n+1} \mid\left(x^{1}, \cdots, x^{n}\right) \in S, a \leq x^{n+1} \leq b\right\} .
$$

We shall prove the following four lemmas, from which Proposition 4.1.1 will follow.

LEMMA 8.1.1. Suppose that $f$ is of class $C^{s}, s \geq 6$, and satisfies the following conditions:

(i) $\left\|\partial f\left(0^{n+1}\right)\right\|=0^{n}$;

(ii) $\left\|\partial^{2} f\left(0^{n+1}\right)\right\|$ is nonsingular and has index $\lambda$.

Then there exists a neighborhood $W$ of $0^{n+1}$ and a diffeomorphism $\Phi \in \Gamma^{s-5}(W)$ with $\Phi\left(0^{n+1}\right)=0^{n+1}$ such that the function $F \circ \Phi=\log f \circ \Phi$ is expressed as

$$
(F \circ \Phi)(x)=g\left(x^{n+1}\right)-\left(x^{1}\right)^{2}-\cdots-\left(x^{2}\right)^{2}+\cdots+\left(x^{n}\right)^{2}, \quad x \in W,
$$

with a suitable function $g$ depending only on $x^{n+1}$.

LEMMA 8.1.2. Suppose that $f$ is of class $C^{s}, s \geq 11$, and has the following properties:

(i ) $\left\|\partial f\left(0^{n+1}\right)\right\|=0^{n}$;

(ii) $\left\|\partial^{2} f\left(0^{n+1}\right)\right\|$ is singular and has index $\mu$;

(iii) $\left\|\partial^{3} f\left(0^{n+1}\right)\right\|$ is nonsingular.

Then there exists a neighborhood $W$ of $0^{n+1}$ and a diffeomorphism $\Phi \in \Gamma^{s-8}(W)$ with $\Phi\left(0^{n+1}\right)=0^{n+1}$ such that the function $F \circ \Phi=\log f \circ \Phi$ is expressed as

$$
(F \circ \Phi)(x)=g\left(x^{n}, x^{n+1}\right)-\left(x^{1}\right)^{2}-\cdots-\left(x^{\mu}\right)^{2}+\cdots+\left(x^{n-1}\right)^{2}, \quad x \in W,
$$

where $g$ is a $C^{s-8}$ function which depends only on $x^{n}, x^{n+1}$ and is "similar" to functions $\left(x^{n}\right)^{3} / 3 \pm x^{n+1} x^{n}$, i.e., satisfies the same conditions (i), (ii) as in Definition 4.1.1.

LEMma 8.1.3. Suppose that $f$ is of class $C^{s}, s \geq 5$, and $F=\log f$ is expressed as

$$
F(x)=g\left(x^{n+1}\right)-\left(x^{1}\right)^{2}-\cdots-\left(x^{\lambda}\right)^{2}+\cdots+\left(x^{n}\right)^{2}, \quad x \in U,
$$


with a suitable function $g$ depending only on $x^{n+1}$. Let $A$ be an open subset of $\boldsymbol{R}^{n}$ containing $0^{n}$, and let $U_{1}, U_{2}$ be open subsets of $U$. Suppose that there are four real numbers $a, b, c, d$ satisfying $a<b<c<d$, such that

$$
A_{a}^{d} \subset U, \quad A_{a}^{b} \subset U_{1}, \quad A_{c}^{d} \subset U_{2} .
$$

Let

$$
\Psi_{i}: U_{i} \rightarrow \Psi_{i}\left(U_{i}\right) \subset \boldsymbol{R}^{n+1}, \quad i=1,2,
$$

be two diffeomorphisms of class $C^{s}$ which are expressed in the forms

$$
\Psi_{i}\left(x^{1}, \cdots, x^{n+1}\right)=\left(\Psi_{i}^{1}(x), \cdots, \Psi_{i}^{n}(x), \Psi_{i}^{n+1}\left(x^{n+1}\right)\right), \quad x \in U_{i},
$$

where $\Psi_{i}^{j}$ are real-valued functions on $U_{i}$, and the functions $\Psi_{i}^{n+1}$ depend only on $x^{n+1}$. Suppose that $\Psi_{i}\left(U_{i} \cap\left(0^{n} \times \boldsymbol{R}\right)\right)=\Psi_{i}\left(U_{i}\right) \cap\left(0^{n} \times \boldsymbol{R}\right), i=1,2$. Suppose that the functions $F \circ\left(\Psi_{i}\right)^{-1}, i=1,2$, are expressed as

$$
F \circ\left(\Psi_{i}\right)^{-1}(x)=g_{i}\left(x^{n+1}\right)-\left(x^{1}\right)^{2}-\cdots-\left(x^{\lambda}\right)^{2}+\cdots+\left(x^{n}\right)^{2}, \quad x \in \Psi_{i}\left(U_{i}\right),
$$

with suitable functions $g_{i}$ depending only on $x^{n+1}$.

Then there exists an open subset $B$ of $A$ containing $0^{n}$ and a diffeomorphism $\Phi \in \Gamma^{s-4}\left(B_{a}^{d}\right)$ such that

$$
\Phi\left(B_{a}^{b}\right) \subset U_{1}, \quad \Phi\left(B_{c}^{d}\right) \subset U_{2},
$$

and

$$
\begin{array}{ll}
\Phi^{-1}\left(x^{1}, \cdots, x^{n+1}\right)=\left(\Psi_{1}^{1}(x), \cdots, \Psi_{1}^{n}(x), x^{n+1}\right), & x \in \Phi\left(B_{a}^{b}\right), \\
\Phi^{-1}\left(x^{1}, \cdots, x^{n+1}\right)=\left(e^{1} \Psi_{2}^{1}(x), \cdots, e^{n} \Psi_{2}^{n}(x), x^{n+1}\right), & x \in \Phi\left(B_{c}^{d}\right),
\end{array}
$$

for suitable $e^{j}= \pm 1$, and such that

$$
(F \circ \Phi)(x)=h\left(x^{n+1}\right)-\left(x^{1}\right)^{2}-\cdots-\left(x^{\lambda}\right)^{2}+\cdots+\left(x^{n}\right)^{2}, \quad x \in B_{a}^{d},
$$

for a suitable function $h$ depending only on $x^{n+1}$.

LEMMA 8.1.4. Suppose that $f$ is of class $C^{s}, s \geq 3$, and $F=\log f$ is expressed as

$$
F(x)=g\left(x^{n}, x^{n+1}\right)-\left(x^{1}\right)^{2}-\cdots-\left(x^{\mu}\right)^{2}+\cdots+\left(x^{n-1}\right)^{2}, \quad x \in U,
$$

where $g$ is a suitable function which depends only on $x^{n}, x^{n+1}$ and is "similar" to functions $\left(x^{n}\right)^{3} / 3 \pm x^{n+1} x^{n}$, i.e., satisfies the same conditions (i), (ii) as in Definition 4.1.1. Then for any point 


$$
p \in\left\{x \in U \cap\left(0^{n-1} \times R^{2}\right) \mid\|\partial F(x)\|=0^{n}\right\}-\left\{0^{n+1}\right\},
$$

there exists an open set $W$ of $U$ containing $p$ and a diffeomorphism $\Phi \in \Gamma^{s-2}(W)$ such that

(i) $\Phi\left(x^{1}, \cdots, x^{n+1}\right)=\left(x^{1}, \cdots, x^{n-1}, \Phi^{n}\left(x^{n}, x^{n+1}\right), x^{n+1}\right), x \in W$, for a suitable function $\Phi^{n}$ depending only on $x^{n}, x^{n+1}$;

(ii) the function $F \circ \Phi$ is expressed as

$$
(F \circ \Phi)(x)=h\left(x^{n+1}\right)-\left(x^{1}\right)^{2}-\cdots-\left(x^{\tilde{\mu}}\right)^{2}+\cdots+\left(x^{n}\right)^{2}, \quad x \in W,
$$

where $h$ is a suitable function depending only on $x^{n+1}$, and $\tilde{\mu}$ is $\mu$ or $\mu+1$.

To prove these lemmas, we need twelve lemmas, 8.1.5-8.1.16. The following two lemmas are easily verified:

LEMMA 8.1.5. Let $W$ be an open subset of $\boldsymbol{R}^{n+1}$, and let $p$ be a point of $W$. Let $\Phi \in \Gamma^{2}(W)$, and put $q=\Phi(p)$. Suppose that $f$ is of class $C^{2}$ and has the following properties:

(i) $\|\partial f(q)\|=0^{n}$;

(ii) $\left\|\partial^{2} f(q)\right\|$ is nonsingular and has index $\lambda$.

Then $F$ and $f^{*}=f \circ \Phi$ have the same properties as $f$, i.e.,

(i) $\|\partial F(q)\|=\left\|\partial f^{*}(p)\right\|=0^{n}$;

(ii) $\left\|\partial^{2} F(q)\right\|,\left\|\partial^{2} f^{*}(p)\right\|$ are nonsingular and have index $\lambda$.

LEMMA 8.1.6. Let $W$ be an open subset of $\boldsymbol{R}^{n+1}$ containing $0^{n+1}$. Let $\Phi$ be an element of $\Gamma^{3}(W)$ satisfying $\Phi\left(0^{n+1}\right)=0^{n+1}$. Suppose that $f$ is of class $C^{3}$ and has the following properties:

(i ) $\left\|\partial f\left(0^{n+1}\right)\right\|=0^{n}$;

(ii) $\left\|\partial^{2} f\left(0^{n+1}\right)\right\|$ is singular and has index $\mu$;

(iii) $\left\|\partial^{3} f\left(0^{n+1}\right)\right\|$ is nonsingular.

Then $F$ and $f^{*}=f \circ \Phi$ have the same properties as $f$, i.e.,

(i ) $\left\|\partial F\left(0^{n+1}\right)\right\|=\left\|\partial f^{*}\left(0^{n+1}\right)\right\|=0^{n}$;

(ii) $\left\|\partial^{2} F\left(0^{n+1}\right)\right\|,\left\|\partial^{2} f^{*}\left(0^{n+1}\right)\right\|$ are singular and have index $\mu$;

(iii) $\left\|\partial^{3} F\left(0^{n+1}\right)\right\|,\left\|\partial^{3} f^{*}\left(0^{n+1}\right)\right\|$ are nonsingular.

LEMMA 8.1.7. Suppose that $f$ satisfies the following conditions:

(i) $f$ is of class $C^{s}, s \geq 2$;

(ii) $\left\|\partial f\left(0^{n+1}\right)\right\|=0^{n}$;

(iii) $\left\|\partial^{2} f\left(0^{n+1}\right)\right\|$ is nonsingular and has index $\lambda$.

Then there exists an open subset $W$ of $U$ containing $0^{n+1}$ and a dif- 
feomorphism $\Phi \in \Gamma^{s-1}(W)$ such that $\Phi\left(0^{n+1}\right)=0^{n+1}$, and such that the function $f^{*}=f \circ \Phi$ satisfies the following conditions:

(i) $\left\{x \in W \mid\left\|\partial f^{*}(x)\right\|=0^{n}\right\}=W \cap\left(0^{n} \times \boldsymbol{R}\right)$;

(ii) for any $x \in W \cap\left(0^{n} \times \boldsymbol{R}\right),\left\|\partial^{2} f^{*}(x)\right\|$ is nonsingular and has index $\lambda$.

Proof. Let $\Psi: U \rightarrow \boldsymbol{R}^{n+1}$ be a $C^{s-1}$ map defined by

$$
\Psi(x)=\left(\partial_{1} f(x), \cdots, \partial_{n} f(x), x^{n+1}\right)
$$

for $x=\left(x^{1}, \cdots, x^{n+1}\right) \in U$. Since $\Psi\left(0^{n+1}\right)=0^{n+1}$, and the Jacobian of $\Psi$ at $0^{n+1}$ is nonzero, by the inverse function theorem there exists an open subset $W$ of $U$ containing $0^{n+1}$ and a diffeomorphism $\Phi \in I^{s-1}(W)$ such that $\Phi\left(0^{n+1}\right)=0^{n+1}$, and $\Psi \circ \Phi$ is the identity map on $W$. Since $\left(\partial_{j} f\right)(\Phi(x))$ $=x^{j}$, we see that $f^{*}=f \circ \Phi$ satisfies the desired condition (i). Furthermore, by Lemma 8.1.5 we see that $\left\|\partial^{2} f^{*}(x)\right\|$ is nonsingular for any $x \in W \cap\left(0^{n} \times R\right)$, and that $\left\|\partial^{2} f^{*}\left(0^{n+1}\right)\right\|$ has index $\lambda$. Hence we conclude that $\left\|\partial^{2} f^{*}(x)\right\|$ has index $\lambda$ for any $x \in W \cap\left(0^{n} \times R\right)$. Lemma 8.1.7 is proved.

LEMMA 8.1.8. Suppose that $f$ satisfies the following conditions:

(i) $f$ is of class $C^{s}, s \geq 5$;

(ii) $\left\|\partial f\left(0^{n+1}\right)\right\|=0^{n}$;

(iii) $\left\|\partial^{2} f\left(0^{n+1}\right)\right\|$ is singular and has index $\mu$;

(iv) $\left\|\partial^{3} f\left(0^{n+1}\right)\right\|$ is nonsingular.

Then there exists an open subset $W$ of $U$ containing $0^{n+1}$ and a diffeomorphism $\Phi \in \Gamma^{s-4}(W)$ such that $\Phi\left(0^{n+1}\right)=0^{n+1}$, and such that the function $f^{*}=f \circ \Phi$ satisfies the following conditions:

(i ) $\left\{x \in W \mid \partial_{i} f^{*}(x)=0, i=1, \cdots, n-1\right\}=W \cap\left(0^{n-1} \times R^{2}\right)$;

(ii) the set $\left\{x \in W\|\| \partial f^{*}(x) \|=0^{n}\right\}$ coincides with either

$$
\left\{\left(0, \cdots, 0, x^{n}, x^{n+1}\right) \in W \mid\left(x^{n}\right)^{2}-x^{n+1}=0\right\}
$$

or

$$
\left\{\left(0, \cdots, 0, x^{n}, x^{n+1}\right) \in W \mid\left(x^{n}\right)^{2}+x^{n+1}=0\right\} ;
$$

(iii) for any $x \in W \cap\left(0^{n-1} \times \boldsymbol{R}^{2}\right)$, the matrix

$$
\left(\left(\partial^{2} f^{*} / \partial x^{i} \partial x^{j}\right)(x) ; i, j=1, \cdots, n-1\right)
$$

is nonsingular and has index $\mu$.

(iv) $\left(\partial^{2} f^{*} /\left(\partial x^{n}\right)^{2}\right)(x) \neq 0$ for $x \in\left\{x \in W \mid\left\|\partial f^{*}(x)\right\|=0^{n}\right\}-\left\{0^{n+1}\right\}$. 
Proof. From the properties (iii), (iv) of $f$, we see that the rank of $\left\|\partial^{2} f\left(0^{n+1}\right)\right\|$ is $n-1$. Hence there is $\Phi^{\prime} \in \Gamma^{\infty}\left(W^{\prime}\right)$, where $W^{\prime}$ is some open set of $\boldsymbol{R}^{n+1}$ containing $0^{n+1}$, such that $\Phi^{\prime}\left(0^{n+1}\right)=0^{n+1}$, and the matrix $\left(\left(\partial^{2} f \circ \dot{\Phi^{\prime}} / \partial x^{i} \partial x^{j}\right)\left(0^{n+1}\right) ; i, j=1, \cdots, n-1\right)$ is nonsingular. Therefore by Lemma 8.1.6, from the first we may assume that

(v) $\left(\left(\partial^{2} f / \partial x^{i} \partial x^{j}\right)\left(0^{n+1}\right) ; i, j=1, \cdots, n-1\right)$ is nonsingular. Now, let $\Psi$ be a $C^{s-1}$ map defined by

$$
\Psi(x)=\left(\partial_{1} f(x), \cdots, \partial_{n-1} f(x), x^{n}, x^{n+1}\right)
$$

for $x=\left(x^{1}, \cdots, x^{n+1}\right) \in U$. From (v) above and the inverse function theorem it follows that $\Psi$ has the inverse function $\varphi$ defined in a neighborhood $W^{\prime}$ of $0^{n+1}$ in $\boldsymbol{R}^{n+1}$. Then $\varphi\left(0^{n+1}\right)=0^{n+1}$ and $\varphi \in \Gamma^{s-1}\left(W^{\prime}\right)$. Furthermore, $\varphi$ is written in the form

$$
\varphi(x)=\left(\varphi^{1}(x), \cdots, \varphi^{n-1}(x), x^{n}, x^{n+1}\right), \quad x \in W^{\prime},
$$

with suitable functions $\varphi^{i}$. Put

$$
h=f \circ \varphi, \quad h_{i j}=\partial^{2} h / \partial x^{i} \partial x^{j}, \quad h_{n n n}=\partial^{3} h /\left(\partial x^{n}\right)^{3} .
$$

Then we have:

Assertion 1. $h_{n n}\left(0^{n+1}\right)=0, h_{n n+1}\left(0^{n+1}\right) \neq 0, h_{n n n}\left(0^{n+1}\right) \neq 0$.

Proof. If $1 \leq i \leq n-1$, then $\left(\partial_{i} f\right)(\varphi(x))=x^{i}$ for $x \in W^{\prime}$. From this it follows that if $1 \leq i \leq n-1$,

$$
h_{i j}(x)=\frac{\partial^{2} f \circ \varphi}{\partial x^{i} \partial x^{j}}(x)=\frac{\partial}{\partial x^{j}}\left(\sum_{k=1}^{n-1} x^{k} \cdot \frac{\partial \varphi^{k}}{\partial x^{i}}\right) \quad \text { on } W^{\prime} .
$$

Hence we see that if $1 \leq i, j \leq n-1$,

$$
h_{i j}(x)=\sum_{k=1}^{n-1} x^{k} \cdot \frac{\partial^{2} \varphi^{k}}{\partial x^{i} \partial x^{j}}+\frac{\partial \varphi^{j}}{\partial x^{i}} \quad \text { on } W^{\prime},
$$

and that if $1 \leq i \leq n-1$,

$$
h_{i n}(x)=\sum_{k=1}^{n-1} x^{k} \cdot \frac{\partial^{2} \varphi^{k}}{\partial x^{i} \partial x^{n}} \quad \text { on } W^{\prime} .
$$

Thus we have

$$
\begin{gathered}
\operatorname{det}\left\|h_{i j}\left(0^{n+1}\right) ; i, j=1, \cdots, n-1\right\| \neq 0, \\
h_{n i}\left(0^{n+1}\right)=0, \quad i=1, \cdots, n-1 .
\end{gathered}
$$


Since $\operatorname{det}\left\|\partial^{2} h\left(0^{n+1}\right)\right\|=0$ by Lemma 8.1.5, we have

$$
h_{n n}\left(0^{n+1}\right)=0 \text {, }
$$

and hence

$$
\left(\frac{\partial}{\partial x^{n}}\right)_{0} \operatorname{det}\left\|\partial^{2} h(x)\right\|=\operatorname{det}\left(h_{i j}\left(0^{n+1}\right), \frac{\partial h_{i n}}{\partial x^{n}}\left(0^{n+1}\right) ; \begin{array}{l}
i \downarrow 1, \cdots, n \\
j \rightarrow 1, \cdots, n-1
\end{array}\right) .
$$

Since $\operatorname{det}\left\|\partial^{3} h\left(0^{n+1}\right)\right\| \neq 0$ by Lemma 8.1 .5 , we conclude that

$$
h_{n n+1}\left(0^{n+1}\right) \neq 0, \quad\left(\frac{\partial}{\partial x^{n}}\right)_{0} \operatorname{det}\left\|\partial^{2} h(x)\right\| \neq 0,
$$

and hence

$$
h_{n n n}\left(0^{n+1}\right) \neq 0 \text {. }
$$

Assertion 1 is proved.

Now, since the function $\left(\partial_{n} h\right)\left(0, \cdots, 0, x^{n}, x^{n+1}\right)$ of $x^{n}, x^{n+1}$ is of class $C^{s-2}$ and satisfies $\partial_{n} h\left(0^{n+1}\right)=0, h_{n n+1}\left(0^{n+1}\right) \neq 0$, by the implicit function theorem, there exists uniquely a $C^{s-2}$ function $\alpha:\left(-\varepsilon^{\prime}, \varepsilon^{\prime}\right) \rightarrow \boldsymbol{R}$ with small $\varepsilon^{\prime}>0$, such that $\alpha(0)=0$ and $\left(\partial_{n} h\right)(0, \cdots, 0, t, \alpha(t))=0$ for $t \in\left(-\varepsilon^{\prime}, \varepsilon^{\prime}\right)$. From Assertion 1 and the formula

$$
\frac{d \alpha}{d t}(t)=-\frac{h_{n n}(0, \cdots 0, t, \alpha(t))}{h_{n n+1}(0, \cdots 0, t, \alpha(t))},
$$

we see that

$$
\frac{d \alpha}{d t}(0)=0, \quad \frac{d^{2} \alpha}{d t^{2}}(0)=-\frac{h_{n n n}(0)}{h_{n n+1}(0)} \neq 0 .
$$

Applying Morse's lemma to the function $\alpha$, and taking $\varepsilon>0$ sufficiently small, we obtain a $C^{s-4}$ diffeomorphism

$$
\theta:(-\varepsilon, \varepsilon) \rightarrow \theta(-\varepsilon, \varepsilon) \subset\left(-\varepsilon^{\prime}, \varepsilon^{\prime}\right)
$$

such that $t^{2}=|\alpha(\theta(t))|$ on $(-\varepsilon, \varepsilon)$. Now we define $\Phi$ by

$$
\Phi(x)=\varphi\left(x^{1}, \cdots, x^{n-1}, \theta\left(x^{n}\right), x^{n+1}\right)
$$

for $x=\left(x^{1}, \cdots, x^{n+1}\right)$ in a sufficiently small neighborhood $W$ of $0^{n+1}$ in $W^{\prime}$. Then it is checked directly that $\Phi: W \rightarrow \Phi(W)$ is the required diffeomorphism. Lemma 8.1 .8 is proved.

LEMMA 8.1.9. Suppose that $F$ satisfies the following conditions: 
(i) $F$ is of class $C^{s}, s \geq 4$;

(ii) $\left\{x \in U \mid\|\partial F(x)\|=0^{n}\right\}=U \cap\left(0^{n} \times R\right)$;

(iii) for every $x \in U \cap\left(0^{n} \times \boldsymbol{R}\right)$, the matrix $\left\|\partial^{2} F(x)\right\|$ is nonsingular and has index $\lambda$;

(iv) there are two real numbers $b, c$ with $b<c$ such that

$$
\left\|\partial^{2} F(x)\right\|=\operatorname{diag}[-2, \cdots,-2,2, \cdots, 2]
$$

for $x \in\left(\bar{U}_{b} \cup \bar{U}^{c}\right) \cap\left(0^{n} \times R\right)$, where

$$
\begin{aligned}
& \bar{U}_{b}=\left\{\left(x^{1}, \cdots, x^{n+1}\right) \in U \mid x^{n+1} \leq b\right\}, \\
& \bar{U}^{c}=\left\{\left(x^{1}, \cdots, x^{n+1}\right) \in U \mid x^{n+1} \geq c\right\} .
\end{aligned}
$$

Then there exists an open subset $W$ of $U$ containing $U \cap\left(0^{n} \times \boldsymbol{R}\right)$ and a diffeomorphism $\Phi \in \Gamma^{s-2}(W)$ such that

(i ) $\Phi(x)=x$ for $x \in W \cap\left(0^{n} \times R\right)$;

(ii) $\Phi(x)=x$ for $x \in W \cap \bar{U}_{b}$;

(iii) $\Phi\left(x^{1}, \cdots, x^{n+1}\right)=\left(e^{1} x^{1}, \cdots, e^{n} x^{n}, x^{n+1}\right)$ for $x \in W \cap \overline{U^{c}}$, where $e^{i}$ are suitable integers satisfying $\left|e^{i}\right|=1$;

(iv) the function $F^{*}=F \circ \Phi$ satisfies

$\left\|\partial^{2} F^{*}(x)\right\|=\operatorname{diag}[-2, \cdots,-2,2, \cdots, 2] \quad$ for $x \in W \cap\left(0^{n} \times \boldsymbol{R}\right)$.

Proof. Since for each $x \in U \cap\left(0^{n} \times R\right)$, the $n \times n$-matrix $\left\|\partial^{2} F(x)\right\|$ is symmetric and nonsingular, one obtain easily a $C^{s-2}$ map

$$
\gamma_{1}: U \cap\left(0^{n} \times \boldsymbol{R}\right) \rightarrow \boldsymbol{R}^{n}
$$

such that

(i ) $\gamma_{1}(x)=(1,0, \cdots, 0)$ for $x \in \bar{U}_{b} \cap\left(0^{n} \times \boldsymbol{R}\right)$,

(ii) $\sum_{i, j=1}^{n}\left(\partial^{2} F / \partial x^{i} \partial x^{j}\right)(x) \gamma_{1}^{i}(x) \gamma_{1}^{j}(x)= \pm 2$ for $x \in U \cap\left(0^{n} \times R\right)$,

where $\gamma_{1}(x)=\left(\gamma_{1}^{1}(x), \cdots, \gamma_{1}^{n}(x)\right)$,

(iii) $\gamma_{1}(x)=( \pm 1,0, \cdots, 0)$ for $x \in \overline{U^{c}} \cap\left(0^{n} \times R\right)$.

Next, considering for each $x \in U \cap\left(0^{n} \times \boldsymbol{R}\right)$ the "orthogonal" subspace to $\gamma_{1}(x)$ one obtain a $C^{s-2}$ map

$$
\gamma_{2}: U \cap\left(0^{n} \times R\right) \rightarrow R^{n}
$$

such that

(i ) $\gamma_{2}(x)=(0,1,0 \ldots 0)$ for $x \in \bar{U}_{b} \cap\left(0^{n} \times R\right)$,

(ii) $\sum_{i, j=1}^{n}\left(\partial^{2} F / \partial x^{i} \partial x^{j}\right)(x) \gamma_{1}^{i}(x) \gamma_{2}^{j}(x)=0$, 


$$
\sum_{i, j=1}^{n}\left(\partial^{2} F / \partial x^{i} \partial x^{j}\right)(x) \gamma_{2}^{i}(x) \gamma_{2}^{j}(x)= \pm 2 \quad \text { for } x \in U \cap\left(0^{n} \times R\right)
$$

where $\gamma_{2}(x)=\left(\gamma_{2}^{1}(x), \cdots, \gamma_{2}^{n}(x)\right)$,

(iii) $\gamma_{2}(x)=(0, \pm 1,0 \cdots 0)$ for $x \in \bar{U}^{c} \cap\left(0^{n} \times R\right)$.

Repeating the process $n$ times, we obtain the $n$ "vector fields" $\gamma_{1}, \cdots, \gamma_{n}$ of class $C^{s-2}$ defined on $U \cap\left(0^{n} \times \boldsymbol{R}\right)$ such that for each $k=1, \cdots, n$,

(i ) $\sum_{i, j=1}^{n}\left(\partial^{2} F / \partial x^{i} \partial x^{j}\right)(x) \gamma_{k}^{i}(x) \gamma_{m}^{j}(x)= \pm 2 \delta_{k m}$ on $U \cap\left(0^{n} \times \boldsymbol{R}\right)$, where $\gamma_{k}(x)=\left(\gamma_{k}^{1}(x), \cdots, \gamma_{k}^{n}(x)\right)$, and $\delta$ is the Kronecker delta,

(ii) $\gamma_{k}(x)=(0, \cdots 0,1,0 \cdots 0)$ (1 is the $k$-th component) for $x \in \bar{U}_{b} \cap\left(0^{n} \times \boldsymbol{R}\right)$,

(iii) $\gamma_{k}(x)=(0, \cdots 0, \pm 1,0 \cdots 0)( \pm 1$ is the $k$-th component) for $x \in \bar{U}^{c} \cap\left(0^{n} \times \boldsymbol{R}\right)$.

Now we define $\Phi: U \rightarrow R^{n+1}$ by

$$
\Phi\left(x^{1}, \cdots, x^{n+1}\right)=\left(x^{1} \gamma_{1}\left(0^{n}, x^{n+1}\right)+\cdots+x^{n} \gamma_{n}\left(0^{n}, x^{n+1}\right), x^{n+1}\right),
$$

and put $W=\Phi^{-1}(U)$. Then it is easily verified that the diffeomorphism $\Phi: W \rightarrow \Phi(W)$ has the required properties.

The following two lemmas are proved similarly.

LEMMA 8.1.10. Suppose that $F$ satisfies the following conditions:

(i) $F$ is of class $C^{s}, s \geq 4$;

(ii) $\left\{x \in U \mid \partial_{i} F(x)=0, i=1, \cdots, n-1\right\}=U \cap\left(0^{n-1} \times R^{2}\right)$;

(iii) for every $x \in U \cap\left(0^{n-1} \times R^{2}\right)$, the matrix

$$
\left(\left(\partial^{2} F / \partial x^{i} \partial x^{j}\right)(x) ; i, j=1, \cdots, n-1\right)
$$

is nonsingular and has index $\mu$.

Then there exists an open subset $W$ of $U$ containing $0^{n+1}$ and $a$ diffeomorphism $\Phi \in \Gamma^{s-2}(W)$ such that

(i ) $\Phi(x)=x$ for $x \in W \cap\left(0^{n-1} \times \boldsymbol{R}^{2}\right)$,

(ii) $\Phi(x)=\left(\Phi^{1}(x), \cdots, \Phi^{n-1}(x), x^{n}, x^{n+1}\right)$ for $x \in W$,

where $\Phi^{i}$ are suitable real-valued functions,

(iii) the function $F \circ \Phi$ satisfies

$$
\left(\left(\partial^{2} F \circ \Phi / \partial x^{i} \partial x^{j}\right)(x) ; i, j=1, \cdots, n-1\right)=\operatorname{diag}[-2, \cdots,-2,2, \cdots 2]
$$

for $x \in W \cap\left(0^{n-1} \times R^{2}\right)$. 
LEMMA 8.1.11. Suppose that $F$ satisfies the same conditions (i), (ii), (iii) as in Lemma 8.1.9 and

(v). there are two real numbers $b, c$ with $b<c$ such that

$\left\|\partial^{2} F(x)\right\|=\operatorname{diag}[-2, \cdots,-2,2, \cdots, 2, \alpha(x)]$ for $x \in \bar{U}_{b} \cap\left(0^{n} \times \boldsymbol{R}\right)$, $\left\|\partial^{2} F(x)\right\|=\operatorname{diag}[-2, \cdots,-2,2, \cdots 2]$ for $x \in \bar{U}^{c} \cap\left(0^{n} \times R\right)$,

where $\bar{U}_{b}, \bar{U}^{c}$ denote the same set as in Lemma 8.1.9, and $\alpha$ is a suitable real-valued function.

Then there exists an open set $W$ of $U$ containing $U \cap\left(0^{n} \times \boldsymbol{R}\right)$ and $a$ diffeomorphism $\Phi \in \Gamma^{s-2}(W)$ such that

(i) $\Phi(x)=x$ for $x \in W \cap\left(0^{n} \times R\right)$,

(ii) $\Phi\left(x^{1}, \cdots, x^{n+1}\right)=\left(x^{1}, \cdots, x^{n-1}, \Phi^{n}\left(x^{n}, x^{n+1}\right), x^{n+1}\right)$ for $x \in \bar{U}_{b}$,

where $\Phi^{n}$ is a suitable real-valued function of $x^{n}, x^{n+1}$,

(iii) $\Phi(x)=x$ for $x \in \bar{U}^{c} \cap W$,

(iv) the function $F^{*}=F \circ \Phi$ satisfies

$$
\left\|\partial^{2} F^{*}(x)\right\|=\operatorname{diag}[-2, \cdots,-2,2, \cdots, 2] \text { for } x \in W \cap\left(0^{n} \times R\right) .
$$

The following two lemmas are well known ([5, p. 14, Lemma 2.3]).

LEMMA 8.1.12. Suppose that $F$ satisfies the following conditions:

(i) $F$ is of class $C^{s}, s \geq 2$;

(ii) $\left\{x \in U \mid\|\partial F(x)\|=0^{n}\right\}=U \cap\left(0^{n} \times R\right)$.

If $U$ is convex in $R^{n+1}$, then

$$
F\left(x^{1}, \cdots, x^{n+1}\right)-F\left(0, \cdots, 0, x^{n+1}\right)=\sum_{i, j=1}^{n} a_{i j}(x) x^{i} x^{j}, \quad x \in U,
$$

where $a_{i j}$ are suitable functions of class $C^{s-2}$ on $U$ and satisfy

$$
a_{i j}=a_{j i}, 2 a_{i j}\left(0, \cdots, 0, x^{n+1}\right)=\left(\partial^{2} F / \partial x^{i} \partial x^{j}\right)\left(0, \cdots, 0, x^{n+1}\right) .
$$

LEMMA 8.1.13. Suppose that $F$ satisfies the following conditions:

(i) $F$ is of class $C^{s}, s \geq 2$;

(ii) $\left\{x \in U \mid \partial_{i} F(x)=0, i=1, \cdots, n-1\right\}=U \cap\left(0^{n-1} \times R^{2}\right)$.

If $U$ is convex in $\boldsymbol{R}^{n+1}$, then

$$
F\left(x^{1}, \cdots, x^{n+1}\right)-F\left(0, \cdots, 0, x^{n}, x^{n+1}\right)=\sum_{i, j=1}^{n-1} a_{i j}(x) x^{i} x^{j}, \quad x \in U,
$$

where $a_{i j}$ are suitable functions of class $C^{s-2}$ on $U$ and satisfy

$$
a_{i j}=a_{j i}, 2 a_{i j}\left(0, \cdots, 0, x^{n}, x^{n+1}\right)=\left(\partial^{2} F / \partial x^{i} \partial x^{j}\right)\left(0, \cdots, 0, x^{n}, x^{n+1}\right) .
$$


The following two lemmas are proved by the same methods as in the proof (iii) of Theorem 4.1 of Morse and Cairns [5, p. 25].

LEMMA 8.1.14. Suppose that there exist real-valued functions $a_{i j}$, $i, j=1, \cdots, n$, which are defined on $U$ and satisfy the following conditions :

(i ) $a_{i j}$ are of class $C^{s}, s \geq 1$;

(ii) $a_{i j}=a_{j i}, i, j=1, \cdots, n$;

(iii) $F\left(x^{1}, \cdots, x^{n+1}\right)-F\left(0, \cdots, 0, x^{n+1}\right)=\sum_{i, j=1}^{n} a_{i j}(x) x^{i} x^{j}, x \in U$;

(iv) $\left(a_{i j}(x) ; i, j=1, \cdots, n\right)=\operatorname{diag}[-1, \cdots,-1,1, \cdots, 1]$ (the number of " -1 " is $\lambda)$ for $x \in U \cap\left(0^{n} \times R\right)$;

(v) there are two real numbers $b, c$ with $b<c$ such that $\left(a_{i j}(x) ; i, j=1, \cdots, n\right)=\operatorname{diag}[-1, \cdots,-1,1, \cdots, 1] \quad$ for $x \in \bar{U}_{b} \cup \bar{U}^{c}$, where $\bar{U}_{b}, \bar{U}^{c}$ are the sets defined in Lemma 8.1.9.

Then there exists an open subset $W$ of $U$ containing $U \cap\left(0^{n} \times R\right)$ and a diffeomorphism $\Phi \in \Gamma^{s}(W)$ such that

(i ) $\Phi(x)=x$ for $x \in W \cap\left(0^{n} \times R\right)$,

(ii) $\Phi(x)=x$ for $x \in W \cap\left(\bar{U}_{b} \cup \bar{U}^{c}\right)$,

(iii) $F \circ \Phi(x)-F \circ \Phi\left(0, \cdots, 0, x^{n+1}\right)=-\left(x^{1}\right)^{2}-\cdots-\left(x^{2}\right)^{2}+\cdots+\left(x^{n}\right)^{2}$ for $x \in W$.

LEMMA 8.1.15. Suppose that there exist real-valued functions $a_{i j}$, $i, j=1, \cdots, n-1$, which are defined on $U$ and satisfy the following conditions :

(i) $a_{i j}$ are of class $C^{s}, s \geq 1$;

(ii) $a_{i j}=a_{j i}, i, j=1, \cdots, n-1$;

(iii) $\quad F\left(x^{1}, \cdots, x^{n+1}\right)-F\left(0, \cdots, 0, x^{n}, x^{n+1}\right)=\sum_{i, j=1}^{n-1} a_{i j}(x) x^{i} x^{j}, x \in U$;

(iv) $\left(a_{i j}(x) ; i, j=1, \cdots, n-1\right)=\operatorname{diag}[-1, \cdots,-1,1, \ldots, 1]$ (the number of “ -1 " is $\mu)$ for $x \in U \cap\left(0^{n-1} \times \boldsymbol{R}^{2}\right)$.

Then there exists an open subset $W$ of $U$ containing $U \cap\left(0^{n-1} \times R^{2}\right)$ and a diffeomorphism $\Phi \in \Gamma^{s}(W)$ such that

(i ) $\Phi(x)=x$ for $x \in W \cap\left(0^{n-1} \times \boldsymbol{R}^{2}\right)$;

(ii) $\Phi(x)=\left(\Phi^{1}(x), \cdots, \Phi^{n-1}(x), x^{n}, x^{n+1}\right)$ for $x \in W$, where $\Phi^{i}$ are suitable real-valued functions;

(iii) $F \circ \Phi(x)-F \circ \Phi\left(0, \cdots, 0, x^{n}, x^{n+1}\right)=-\left(x^{1}\right)^{2}-\cdots-\left(x^{\mu}\right)^{2}+\cdots$ $+\left(x^{n-1}\right)^{2}$ for $x \in W$.

LemMa 8.1.16. Let $A$ be an open subset of $\boldsymbol{R}^{n}$ containing $0^{n}$, and 
let $a, b, c, d$ be four real numbers satisfying $a<b<c<d$. Suppose that $A_{a}^{d} \subset U$. Let $U_{1}, U_{2}$ be two open subsets of $U$ such that $A_{a}^{b} \subset U_{1}$, $A_{c}^{d} \subset U_{2}$. Let two diffeomorphisms $\Phi_{i} \in \Gamma^{s}\left(U_{i}\right), i=1,2, s \geq 2$, be given and satisfy

$$
\Phi_{i}(x)=x \quad \text { for } x \in U_{i} \cap\left(0^{n} \times R\right) .
$$

Then there exists an open subset $B$ of $A$ containing $0^{n}$ and $a$ diffeomorphism $\Phi \in \Gamma^{s}\left(B_{a}^{d}\right)$ such that

(i ) $\Phi(x)=x$ for $x \in B_{a}^{d} \cap\left(0^{n} \times \boldsymbol{R}\right)$,

(ii) $\Phi(x)=\Phi_{1}(x)$ for $x \in B_{a}^{b}$,

(iii) $\Phi(x)=\left(e^{1} \Phi_{2}^{1}(x), \cdots, e^{n} \Phi_{2}^{n}(x), x^{n+1}\right)$ for $x \in B_{c}^{d}$,

where $\Phi_{2}(x)=\left(\Phi_{2}^{1}(x), \cdots, \Phi_{2}^{n}(x), x^{n+1}\right)$, and $e^{i}$ are suitable integers satisfying $\left|e^{i}\right|=1$.

Proof. Using the uniqueness theorem of tubular neighborhood (Lang [2, p. 77]), we find easily an open set $B^{\prime}$ of $A$ and a diffeomorphism $\Phi^{\prime} \in \Gamma^{s-1}\left(\left(B^{\prime}\right)_{a}^{d}\right)$ satisfying the conditions (i)-(iii) above. Smoothing this $\Phi^{\prime}$, we obtain the desired diffeomorphism $\Phi \in \Gamma^{s}\left(B_{a}^{d}\right)$ with an open subset $B$ of $A$ containing $0^{n}$. Lemma 8.1.16 is proved.

Proof of Lemma 8.1.1. For the proof, we denote by $\Gamma^{s^{\prime}}\left(W^{\prime}, U^{\prime}\right)$, where $s^{\prime}$ is a positive integer, and $W^{\prime}, U^{\prime}$ are subsets of $\boldsymbol{R}^{n+1}$, the set of all $C^{s^{\prime}}$ diffeomorphisms $\Phi: W^{\prime} \rightarrow \Phi\left(W^{\prime}\right) \subset U^{\prime}$ which are written in the form

$$
\Phi\left(x^{1}, \cdots, x^{n+1}\right)=\left(\Phi^{1}(x), \cdots, \Phi^{n}(x), x^{n+1}\right)
$$

with suitable real-valued functions $\Phi^{i}$ defined on $W^{\prime}$.

Now, for the function $f: U \rightarrow R$, by Lemma 8.1.7, there exists an open subset $W_{1}$ of $U$ containing $0^{n+1}$ and a diffeomorphism $\Phi_{1} \in \Gamma^{s-1}\left(W_{1}, U\right)$ with $\Phi_{1}\left(0^{n+1}\right)=0^{n+1}$, such that the function $f_{1}=f \circ \Phi_{1}$ satisfies

(i) $\left\{x \in W_{1} \mid\left\|\partial f_{1}(x)\right\|=0^{n}\right\}=W_{1} \cap\left(0^{n} \times \boldsymbol{R}\right)$;

(ii) for any $x \in W_{1} \cap\left(0^{n} \times R\right)$, the matrix $\left\|\partial^{2} f_{1}(x)\right\|$ is nonsingular, with index $\lambda$.

By Lemma 8.1.5, we see that the function $F_{1}=\log f_{1}$ satisfies the same conditions (i), (ii) as $f_{1}$. Applying Lemma 8.1.9 to the $C^{s-1}$ function $F_{1}: W_{1} \rightarrow \boldsymbol{R}$ (it being understood that the sets $\overline{\left(W_{1}\right)_{b}}, \overline{\left(W_{1}\right)^{c}}$ are empty), we obtain an open subset $W_{2}$ of $W_{1}$ containing $0^{n+1}$ and a diffeomorphism $\Phi_{2} \in \Gamma^{s-3}\left(W_{2}, W_{1}\right)$ such that $\Phi_{2}(x)=x$ for $x \in W_{2} \cap\left(0^{n} \times \boldsymbol{R}\right)$, and such that the function $F_{2}=F_{1} \circ \Phi_{2}$ satisfies 


$$
\left\|\partial^{2} F_{2}(x)\right\|=\operatorname{diag}[-2, \cdots,-2,2, \cdots, 2] \quad \text { for } x \in W_{2} \cap\left(0^{n} \times R\right) .
$$

Using Lemma 8.1.5 again, and applying Lemma 8.1.12 to the $C^{s-3}$ function $F_{2}: W_{2} \rightarrow \boldsymbol{R}$, we see that for a suitable open subset $W_{3}$ of $W_{2}$ containing $0^{n+1}$, the function $F_{2}$ is written as

$$
F_{2}(x)-F_{2}\left(0, \cdots, 0, x^{n+1}\right)=\sum_{i, j=1}^{n} a_{i j}(x) x^{i} x^{j}, \quad x \in W_{3},
$$

where $a_{i j}$ are suitable functions of class $C^{s-5}$ satisfying

$$
\begin{array}{r}
a_{i j}=a_{j i},\left(a_{i j}(x) ; i, j=1, \cdots, n\right)=\operatorname{diag}[-1, \cdots,-1,1, \cdots, 1] \\
\text { for } x \in W_{3} \cap\left(0^{n} \times R\right) .
\end{array}
$$

Hence, the function $F_{2}: W_{3} \rightarrow \boldsymbol{R}$ satisfies the assumption of Lemma 8.1.14, with the $C^{s-5}$ functions $a_{i j}$ (it being understood that the sets $\overline{\left(W_{3}\right)_{b}}, \overline{\left(W_{3}\right)^{c}}$ are empty). Thus there exists an open set $W_{4}$ of $W_{3}$ containing $0^{n+1}$ and a diffeomorphism $\Phi_{4} \in \Gamma^{s-5}\left(W_{4}, W_{3}\right)$ such that

(i ) $\Phi_{4}(x)=x$ for $x \in W_{4} \cap\left(0^{n} \times R\right)$;

(ii ) $F_{2} \circ \Phi_{4}(x)-F_{2} \circ \Phi_{4}\left(0, \cdots, 0, x^{n+1}\right)=-\left(x^{1}\right)^{2}-\cdots-\left(x^{\lambda}\right)^{2}+\cdots+\left(x^{n}\right)^{2}$ for $x \in W_{4}$.

Consequently we olserve that the diffeomorphism $\Phi=\Phi_{1} \circ \Phi_{2} \circ \Phi_{4} \epsilon$ $\Gamma^{s-5}\left(W_{4}, U\right)$ satisfies the following properties:

(i ) $\Phi\left(0^{n+1}\right)=0^{n+1}$;

(ii) $\log f \circ \Phi(x)=F_{2} \circ \Phi_{4}\left(0, \cdots, 0, x^{n+1}\right)-\left(x^{1}\right)^{2}-\cdots+\left(x^{n}\right)^{2}$ for $x \in W_{4}$. This proves Lemma 8.1.1.

Proof of Lemma 8.1.2. Similarly to the proof of Lemma 8.1.1, using Lemmas 8.1.6, 8, 10, 13 and 15, one proves Lemma 8.1.2.

Proof of Lemma 8.1.3. We use the same notation $\Gamma^{s^{\prime}}\left(W^{\prime}, U^{\prime}\right)$ as in the proof of Lemma 8.1.1. For the diffeomorphisms $\Psi_{i}, i=1,2$, we consider diffeomorphisms $\Phi_{i}$ defined by

$$
\Phi_{i}\left(x^{1}, \cdots, x^{n+1}\right)=\left(\Psi_{i}^{1}(x), \cdots, \Psi_{i}^{n}(x), x^{n+1}\right), \quad x \in U_{i} .
$$

Let $U_{i}^{\prime}, i=1,2$, be open subsets of $U_{i}$ containing $U_{i} \cap\left(0^{n} \times R\right)$ such that $\Phi_{i}\left(U_{i}^{\prime}\right) \subset U$. Then $\Phi_{i} \in \Gamma^{s}\left(U_{i}^{\prime}, U\right)$. Let $\varepsilon$ be a sufficiently small positive number, and let $A^{\prime}$ be an open subset of $A$ containing $0^{n}$ such that $\left(A^{\prime}\right)_{a-\varepsilon}^{b} \subset U_{1}^{\prime},\left(A^{\prime}\right)_{c}^{d+\varepsilon} \subset U_{2}^{\prime}$. Applying Lemma 8.1.16 to $\Phi_{i}$, we obtain an open subset $C$ of $A^{\prime}$ containing $0^{n}$ and a diffeomorphism $\Theta \in \Gamma^{s}\left(C_{a-s}^{d+\varepsilon}\right)$ such that 
(i ) $\Theta(x)=x$ for $x \in C_{a-\varepsilon}^{d+s} \cap\left(0^{n} \times \boldsymbol{R}\right)$,

(ii) $\Theta(x)=\left(\Psi_{1}^{1}(x), \cdots, \Psi_{1}^{n}(x), x^{n+1}\right)$ for $x \in C_{a-s}^{b}$,

(iii) $\Theta(x)=\left(e^{1} \Psi_{2}^{1}(x), \cdots, e^{n} \Psi_{2}^{n}(x), x^{n+1}\right)$ for $x \in C_{c}^{d+\varepsilon}, e^{i}= \pm 1$.

Using the relations

$$
\begin{array}{ll}
\left(\Psi_{1} \circ \Theta^{-1}\right)(x)=\left(x^{1}, \cdots, x^{n}, \Psi_{1}^{n+1}\left(x^{n+1}\right)\right), & x \in \Theta\left(C_{a-\varepsilon}^{b}\right), \\
\left(\Psi_{2} \circ \Theta^{-1}\right)(x)=\left(e^{1} x^{1}, \cdots, e^{n} x^{n}, \Psi_{2}^{n+1}\left(x^{n+1}\right)\right), & x \in \Theta\left(C_{c}^{d+\varepsilon}\right),
\end{array}
$$

we observe that for $x \in \Theta\left(C_{a-\varepsilon}^{b}\right)$,

$$
\begin{aligned}
F \circ \Theta^{-1}(x) & =\left(F \circ\left(\Psi_{1}\right)^{-1} \circ \Psi_{1} \circ \Theta^{-1}\right)(x) \\
& =\left(F \circ\left(\Psi_{1}\right)^{-1}\right)\left(x^{1}, \cdots, x^{n}, \Psi_{1}^{n+1}\left(x^{n+1}\right)\right) \\
& =\tilde{g}_{1}\left(x^{n+1}\right)-\left(x^{1}\right)^{2}-\cdots+\left(x^{n}\right)^{2},
\end{aligned}
$$

where $\tilde{g}_{1}$ is a suitable function depending only on $x^{n+1}$, and that for $x \in \Theta\left(C_{c}^{d+\varepsilon}\right)$,

$$
\begin{aligned}
F \circ \Theta^{-1}(x) & =\left(F \circ\left(\Psi_{2}\right)^{-1} \circ \Psi_{2} \circ \Theta^{-1}\right)(x) \\
& =\left(F \circ\left(\Psi_{2}\right)^{-1}\right)\left(e^{1} x^{1}, \cdots, e^{n} x^{n}, \Psi_{2}^{n+1}\left(x^{n+1}\right)\right) \\
& =\tilde{g}_{2}\left(x^{n+1}\right)-\left(x^{1}\right)^{2}-\cdots+\left(x^{n}\right)^{2},
\end{aligned}
$$

where $\tilde{g}_{2}$ is a suitable function depending only on $x^{n+1}$.

Applying Lemma 8.1.9 to the $C^{s}$ function $F \circ \Theta^{-1}: \Phi\left(\operatorname{Int} C_{a-\varepsilon}^{d+\varepsilon}\right) \rightarrow \boldsymbol{R}$, we obtain a convex, open subset $D$ of $C$ containing $0^{n}$ and a diffeomorphism $\Lambda \in \Gamma^{s-2}\left(D_{\alpha}^{\delta}, \Theta\left(C_{a-\varepsilon}^{d+\varepsilon}\right)\right)$, where $\alpha, \beta$ are suitable numbers satisfying $a-\varepsilon<$ $\alpha<a, d<\delta<d+\varepsilon$, such that

(i ) $\Lambda(x)=x$ for $x \in D_{\alpha}^{\delta} \cap\left(0^{n} \times R\right)$,

(ii) $\Lambda(x)=x$ for $x \in D_{\alpha}^{b}$,

(iii) $\Lambda(x)=\left(\dot{e}^{1} x^{1}, \cdots, \dot{e}^{n} x^{n}, x^{n+1}\right)$ for $x \in D_{c}^{\delta}$,

where $\dot{e}^{i}$ are suitable integers satisfying $\left|\dot{e}^{i}\right|=1$,

(iv) the function $F^{*}=F \circ \Theta^{-1} \circ \Lambda$ satisfies

$$
\left\|\partial^{2} F^{*}(x)\right\|=\operatorname{diag}[-2, \cdots,-2,2, \cdots, 2] \quad \text { for } x \in D_{\alpha}^{\delta} \cap\left(0^{n} \times R\right) \text {. }
$$

From Lemma 8.1.12, it follows that the $C^{s-2}$ function $F^{*}=F \circ \Theta^{-1} \circ \Lambda$ is written as

$$
F^{*}\left(x^{1}, \cdots, x^{n+1}\right)-F^{*}\left(0, \cdots, 0, x^{n+1}\right)=\sum_{i, j=1}^{n} a_{i j}(x) x^{i} x^{j}, \quad x \in C_{\alpha}^{\delta},
$$

where $a_{i f}$ are suitable functions of class $C^{s-4}$ and have the following properties: 
(i ) $a_{i j}=a_{j i}$;

(ii) $\left(a_{i j}\left(0, \cdots, 0, x^{n+1}\right) ; i, j=1, \cdots, n\right)=\operatorname{diag}[-1, \cdots,-1,1, \cdots, 1]$;

(iii) $\left(a_{i j}(x) ; i, j=1, \cdots, n\right)=\operatorname{diag}[-1, \cdots,-1,1, \cdots, 1]$ for $x \in D_{\alpha}^{b}$ $\cup D_{c}^{\delta}$.

By Lemma 8.1.14, we find an open subset $B$ of $D$ containing $0^{n}$ and a diffeomorphism $\Xi \in \Gamma^{s-4}\left(B_{a}^{d}, D_{\alpha}^{\delta}\right)$ such that

(i ) $\Xi(x)=x$ for $x \in B_{a}^{d} \cap\left(0^{n} \times R\right)$,

(ii) $\Xi(x)=x$ for $x \in B_{a}^{b} \cup B_{c}^{d}$,

(iii) $F^{*} \circ \Xi(x)-F^{*} \circ \Xi\left(0, \cdots, 0, x^{n+1}\right)=-\left(x^{1}\right)^{2}-\cdots-\left(x^{\lambda}\right)^{2}+\cdots+\left(x^{n}\right)^{2}$

for $x \in B_{a}^{d}$.

Now, we put $\Phi=\Theta^{-1} \circ \Lambda \circ \Xi$. Then $\Phi \in \Gamma^{s-4}\left(B_{a}^{d}, U\right)$. It is obvious that $\Phi\left(B_{a}^{b}\right) \subset C_{a}^{b} \subset U_{1}, \Phi\left(B_{c}^{d}\right) \subset C_{c}^{d} \subset U_{2}$, and that

$$
F \circ \Phi(x)=F^{*} \circ \Xi(x)=F^{*} \circ \Xi\left(0, \cdots, 0, x^{n+1}\right)-\left(x^{1}\right)^{2}-\cdots+\left(x^{n}\right)^{2}
$$

for $x \in B_{a}^{d}$.

From the relations

$$
\Lambda \circ \Xi(x)=x \quad \text { on } B_{a}^{b}, \quad \Lambda \circ \Xi(x)=\left( \pm x^{1}, \cdots, \pm x^{n}, x^{n+1}\right) \quad \text { on } B_{c}^{d},
$$

and the properties

$$
\begin{aligned}
& \Theta(x)=\left(\Psi_{1}^{1}(x), \cdots, \Psi_{1}^{n}(x), x^{n+1}\right) \quad \text { for } x \in \Phi\left(B_{a}^{b}\right), \\
& \Theta(x)=\left( \pm \Psi_{2}^{1}(x), \cdots, \pm \Psi_{2}^{n}(x), x^{n+1}\right) \quad \text { for } x \in \Phi\left(B_{c}^{d}\right)
\end{aligned}
$$

of $\Theta$, we know that

$$
\begin{array}{ll}
\Phi^{-1}\left(x^{1}, \cdots, x^{n+1}\right)=\left(\Psi_{1}^{1}(x), \cdots, \Psi_{1}^{n}(x), x^{n+1}\right), & x \in \Phi\left(B_{a}^{b}\right), \\
\Phi^{-1}\left(x^{1}, \cdots, x^{n+1}\right)=\left( \pm \Psi_{2}^{1}(x), \cdots, \pm \Psi_{2}^{n}(x), x^{n+1}\right), & x \in \Phi\left(B_{c}^{d}\right) .
\end{array}
$$

Consequently, the diffeomorphism $\Phi$ satisfies the required properties. Lemma 8.1.3 is proved.

Proof of Lemma 8.1.4. It is easily verified.

8.2. Proof of Proposition 4.1.1. The proof will be preceded by some definitions and three lemmas. We shall say that an $\mathscr{F}$-chart $\left(U, f ; x^{1}, \cdots, x^{n+1}\right)$ of type $(\lambda), \lambda=0, \cdots, n$, is regular if there is an

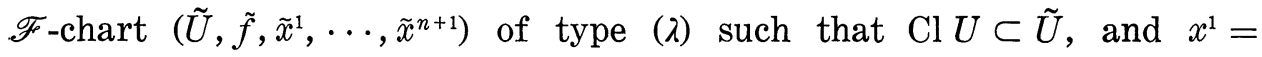
$\tilde{x}^{1}, \cdots, x^{n+1}=\tilde{x}^{n+1}$ on $U$, and such that $U$ is mapped by $\varphi=\left(\tilde{x}^{1}, \cdots, \tilde{x}^{n+1}\right)$ onto an open set of the form $B \times(a, b)$, of $\boldsymbol{R}^{n+1}$, where $B$ is an open subset of $\boldsymbol{R}^{n}$, and $a, b$ are real numbers. We shall say that an $\mathscr{F}$-chart 
$\left(U, f ; x^{1}, \cdots, x^{n+1}\right)$ of type $(*, \mu), \mu=0, \cdots, n-1$, is regular if there is an $\mathscr{F}$-chart $\left(\tilde{U}, \tilde{f} ; \tilde{x}^{1}, \cdots, \tilde{x}^{n+1}\right)$ of type $(*, \mu)$ which has the same properties as above and in addition, satisfies the following condition: $U$ is mapped by $\varphi$ onto an open set of the form $C \times(-2 c, 2 c) \times\left(-c^{2}, c^{2}\right)$ of $R^{n+1}$, where $C$ is an open subset of $\boldsymbol{R}^{n-1}$, and $c$ is a positive real number. Recall that an $\mathscr{F}$-chart $\left(U, f ; x^{1}, \cdots, x^{n+1}\right)$ is said to be of class $C^{s}(1 \leq s \leq r+1)$ if $\left(x^{1}, \cdots, x^{n+1}\right)$ is of class $C^{s}$ (and $f$ is of class $C^{s-1}$ ), and recall that $\omega$ is assumed to be of class $C^{r}, r \geq 21$.

Lemma 8.2.1. Assume that $\omega$ satisfies Condition (T). Then:

(i) For any point $p$ of $\Sigma_{\lambda}, 0 \leq \lambda \leq n$, there exists an $\mathscr{F}$-chart at $p$, of class $C^{r-6}$ and of type ( $\lambda$ ) and with regularity.

(ii) For any point $p$ of $\Sigma_{*}$, there exists an $\mathscr{F}$-chart at $p$, of class $C^{r-9}$ and of type $(*, \mu)$ for some $\mu=0,1, \cdots, n-1$.

Proof. (i) For $p \in \Sigma_{\lambda}$, there is an $\mathscr{F}$-chart $\left(U, f ; x^{1}, \cdots, x^{n+1}\right)$ at $p$, of class $C^{r}$ such that $x^{1}(p)=\cdots=x^{n+1}(p)=0$. Let $\varphi: U \rightarrow \varphi(U) \subset \boldsymbol{R}^{n+1}$ be a $C^{r}$ diffeomorphism defined by the local coordinate system $\left(x^{1}, \cdots, x^{n+1}\right)$. Applying Lemma 8.1.1 to a function $f \circ \varphi^{-1}$ defined on $\varphi(U)$, we obtain an open subset $W$ of $\varphi(U)$ containing the origin $0^{n+1}$ of $\boldsymbol{R}^{n+1}$ and a $C^{r-6}$ diffeomorphism $\Phi: W \rightarrow \Phi(W) \subset \varphi(U)$ such that $\Phi\left(0^{n+1}\right)=0^{n+1}, \Phi^{*}\left(d x^{n+1}\right)=$ $d x^{n+1}$ on $W$, and such that

$$
\log f \circ \varphi^{-1} \circ \Phi(x)=g\left(x^{n+1}\right)-\left(x^{1}\right)^{2}-\cdots+\left(x^{n}\right)^{2}, \quad x \in W,
$$

for a suitable function $g$ depending only on $x^{n+1}$. Then taking a suitable open subset of $\varphi^{-1}(\Phi(W))$, we observe that the restriction to it of the diffeomorphism $\Phi^{-1} \circ \varphi: \varphi^{-1}(\Phi(W)) \rightarrow W \subset \boldsymbol{R}^{n+1}$ defines an $\mathscr{F}$-chart at $p$, of class $C^{r-6}$ and of type ( $\lambda$ ), and with regularity. This proves the part (i). Using Lemma 8.1.2, one proves (ii) similarly. Lemma 8.2.1 is proved.

LEMMA 8.2.2. Let $\lambda$ be an integer on the range $0,1, \cdots, n$. Suppose that three $\mathscr{F}$-charts $\left(U, f ; x^{1}, \cdots, x^{n+1}\right),\left(U_{i}, f_{i} ; x_{i}^{1}, \cdots, x_{i}^{n+1}\right), i=1,2$, of

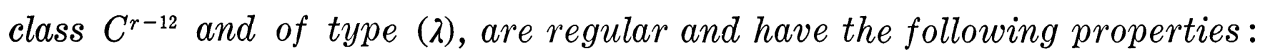

$$
U \cap U_{i} \cap \Sigma \neq \emptyset, \quad U_{i} \cap \Sigma \not \subset U, \quad \mathrm{Cl} U_{1} \cap \mathrm{Cl} U_{2}=\emptyset, \quad i=1,2 .
$$

Then there exists an $\mathscr{F}$-chart $\left(W, h ; y^{1}, \cdots, y^{n+1}\right)$ of class $C^{r-17}$ and of type (ג) and with regularity, such that $U \cap \Sigma \subset W$, and 


$$
\begin{array}{ll}
\left(y^{1}, \cdots, y^{n}\right)=\left(x_{1}^{1}, \cdots, x_{1}^{n}\right) & \text { on } W \cap U_{1}, \\
\left(y^{1}, \cdots, y^{n}\right)=\left(e^{1} x_{2}^{1}, \cdots, e^{n} x_{2}^{n}\right) & \text { on } W \cap U_{2}
\end{array}
$$

for suitable $e^{i}= \pm 1$.

Proof. Let $\left(\tilde{U}, \tilde{f} ; \tilde{x}^{1}, \cdots, \tilde{x}^{n+1}\right),\left(\tilde{U}_{i}, \tilde{f}_{i} ; \tilde{x}_{i}^{1}, \cdots, \tilde{x}_{i}^{n+1}\right)$ be the "extensions" of the regular $\mathscr{F}$-charts $\left(U, f ; x^{1}, \cdots, x^{n+1}\right),\left(U_{i}, f_{i} ; x_{i}^{1}, \cdots, x_{i}^{n+1}\right), i=1,2$, respectively. Let

$$
\varphi: \tilde{U} \rightarrow \varphi(\tilde{U}), \quad \varphi_{i}: \tilde{U}_{i} \rightarrow \varphi_{i}\left(\tilde{U}_{i}\right)
$$

be the $C^{r-12}$ diffeomorphisms defined by the local coordinate systems $\left(\tilde{x}^{1}, \cdots, \tilde{x}^{n+1}\right),\left(\tilde{x}_{i}^{1}, \cdots, \tilde{x}_{i}^{n+1}\right)$, respectively. Now, to apply Lemma 8.1 .3 , we consider two diffeomorphisms of class $C^{r-12}$

$$
\begin{aligned}
& \Psi_{1}=\varphi_{1} \circ \varphi^{-1}: \varphi\left(\tilde{U} \cap \tilde{U}_{1}\right) \rightarrow \varphi_{1}\left(\tilde{U} \cap \tilde{U}_{1}\right), \\
& \Psi_{2}=\varphi_{2} \circ \varphi^{-1}: \varphi\left(\tilde{U} \cap \tilde{U}_{2}\right) \rightarrow \varphi_{2}\left(\tilde{U} \cap \tilde{U}_{2}\right) .
\end{aligned}
$$

It is clear that $\Psi_{i}\left(\varphi\left(\tilde{U} \cap \tilde{U}_{i}\right) \cap\left(0^{n} \times \boldsymbol{R}\right)\right)=\left(\Psi_{i} \circ \varphi\right)\left(\tilde{U} \cap \tilde{U}_{i}\right) \cap\left(0^{n} \times \boldsymbol{R}\right)$, where $0^{n}$ denotes the origin of $\boldsymbol{R}^{n}$, and the product $0^{n} \times \boldsymbol{R}$ is to be understood as the subspace of $\boldsymbol{R}^{n+1}$. Moreover, it is obvious that $\Psi_{i}$ are written in the form

$$
\Psi_{i}(x)=\left(\Psi_{i}^{1}(x), \cdots, \Psi_{i}^{n}(x), \Psi_{i}^{n+1}\left(x^{n+1}\right)\right), \quad x=\left(x^{1}, \cdots, x^{n+1}\right) \in \varphi\left(\tilde{U} \cap \tilde{U}_{i}\right),
$$

where $\Psi_{i}^{j}$ are real-valued functions, and $\Psi_{i}^{n+1}$ depend only on $x^{n+1}$. Hence we have

$$
\left(\Psi_{i}^{-1}\right) * d x^{n+1}=z_{i}\left(x^{n+1}\right) d x^{n+1} \quad \text { on } \varphi_{i}\left(\tilde{U} \cap \tilde{U}_{i}\right)
$$

where $z_{i}$ are suitable functions depending only on $x^{n+1}$. From the relations

$$
\left(\tilde{f}_{i} \circ \varphi_{i}^{-1}\right) d x^{n+1}=\left(\tilde{f} \circ \varphi_{i}^{-1}\right)\left(\left(\Psi_{i}^{-1}\right)^{*} d x^{n+1}\right) \quad \text { on } \varphi_{i}\left(\tilde{U} \cap \tilde{U}_{i}\right),
$$

we see that the functions $\log \tilde{f}_{i} \circ \varphi_{i}^{-1}-\log \tilde{f} \circ \varphi_{i}^{-1}: \varphi_{i}\left(\tilde{U} \cap \tilde{U}_{i}\right) \rightarrow \boldsymbol{R}$ depend only on $x^{n+1}$. Hence the functions $\log \tilde{f} \circ \varphi^{-1} \circ \Psi_{i}^{-1}$ are written as

$$
\log \tilde{f} \circ \varphi^{-1} \circ \Psi_{i}^{-1}(x)=g_{i}\left(x^{n+1}\right)-\left(x^{1}\right)^{2}-\cdots+\left(x^{n}\right)^{2}, \quad x \in \varphi_{i}\left(\tilde{U} \cap \tilde{U}_{i}\right),
$$

with suitable functions $g_{i}$ depending only on $x^{n+1}$. On the other hand, by definition, the set $\varphi(U)$ is written in the form

$$
\varphi(U)=\tilde{A} \times(a, d) \subset \boldsymbol{R}^{n+1},
$$


where $\tilde{A}$ is an open subset of $\boldsymbol{R}^{n}$ containing the origin $0^{n}$, and $a, d$ are real numbers. It is clear that $\varphi(U \cap \Sigma)=0^{n} \times(a, d)$. It is easily verified that the point $0^{n} \times a$ of $\boldsymbol{R}^{n+1}$ is contained in either $\varphi\left(\tilde{U} \cap U_{1} \cap \Sigma\right)$ or $\varphi\left(\tilde{U} \cap U_{2} \cap \Sigma\right)$, say $0^{n} \times a \in \varphi\left(\tilde{U} \cap U_{1} \cap \Sigma\right)$. Then $0^{n} \times d$ is contained in $\varphi\left(\tilde{U} \cap U_{2} \cap \Sigma\right)$, and there are two real numbers $b, c$ satisfying $a<b<$ $c<d$, such that

$$
\varphi\left(U \cap U_{1} \cap \Sigma\right)=0^{n} \times(a, b), \quad \varphi\left(U \cap U_{2} \cap \Sigma\right)=0^{n} \times(c, d) .
$$

Then we can find a bounded, open subset $A$ of $\boldsymbol{R}^{n}$ such that

$$
0^{n} \in A \subset \mathrm{Cl} A \subset \tilde{A},
$$

and

$$
\text { Cl } A \times(a, b) \subset \varphi\left(U \cap U_{1}\right), \quad \text { Cl } A \times(c, d) \subset \varphi\left(U \cap U_{2}\right) .
$$

For this $A$, there is a positive number $\varepsilon$ such that

$$
A \times[a-\varepsilon, b] \subset \varphi\left(\tilde{U} \cap \tilde{U}_{1}\right), \quad A \times[c, d+\varepsilon] \subset \varphi\left(\tilde{U} \cap \tilde{U}_{2}\right) .
$$

Now, applying Lemma 8.1.3 to the $C^{r-13}$ function $\tilde{f} \circ \varphi^{-1}: \varphi(\tilde{U}) \rightarrow \boldsymbol{R}$ and the $C^{r-12}$ diffeomorphisms $\Psi_{i}: \varphi\left(\tilde{U} \cap \tilde{U}_{i}\right) \rightarrow \varphi_{i}\left(\tilde{U} \cap \tilde{U}_{i}\right)$, we obtain an open subset $B$ of $A$ containing $0^{n}$ and a $C^{r-17}$ diffeomorphism

$$
\Phi: B_{a-\varepsilon}^{d+\varepsilon} \rightarrow \Phi\left(B_{a-\varepsilon}^{d+\varepsilon}\right) \subset \boldsymbol{R}^{n+1}
$$

(with the notation of 8.1 ) such that

$$
\begin{array}{ll}
\Phi^{*}\left(d x^{n+1}\right)=d x^{n+1} \quad \text { on } B_{a-\varepsilon}^{d+\varepsilon}, & \\
\Phi^{-1}(x)=\left(\Psi_{1}^{1}(x), \cdots, \Psi_{1}^{n}(x), x^{n+1}\right) & \text { for } x \in B_{a-\varepsilon}^{b}, \\
\Phi^{-1}(x)=\left(e^{1} \Psi_{2}^{1}(x), \cdots, e^{n} \Psi_{2}^{n}(x), x^{n+1}\right) & \text { for } x \in B_{c}^{d+\varepsilon}, e^{j}= \pm 1,
\end{array}
$$

and such that

$$
\log \tilde{f} \circ \varphi^{-1} \circ \Phi(x)=h_{0}\left(x^{n+1}\right)-\left(x^{1}\right)^{2}-\cdots+\left(x^{n}\right)^{2} \quad \text { on } B_{a-\varepsilon}^{d+\varepsilon} .
$$

It is easily verified that the restriction of the diffeomorphism $\Phi^{-1} \circ \varphi: \varphi^{-1}\left(B_{a-\varepsilon}^{d+\varepsilon}\right) \rightarrow \Phi^{-1}\left(B_{a-\varepsilon}^{d+\varepsilon}\right)$ to a suitable open subset $W$ of $\tilde{U}$, gives us the required $\mathscr{F}$-chart. Lemma 8.2.2 is proved.

Using Lemma 8.1.4, one proves easily the following lemma.

LEMMA 8.2.3. Let $\mu$ be an integer on the range $0,1, \cdots, n-1$. Suppose that an $\mathscr{F}$-chart $\left(U, f ; x^{1}, \cdots, x^{n+1}\right)$ of class $C^{r-9}$ and of type $(*, \mu)$ and with regularity, is given. For any point $p$ of $U \cap\left(\Sigma-\Sigma_{*}\right)$, 
there exists an F -chart-at-p $\left(W, h ; y^{1}, \cdots, y^{n+1}\right)$ which is of class $C^{r-12}$ and of type $(\mu)$ or type $(\mu+1)$, and is regular, such that

$$
\left(x^{1}, \cdots, x^{n-1}\right)=\left(y^{1}, \cdots, y^{n-1}\right), \quad x^{n}=\Phi\left(y^{n}, y^{n+1}\right) \quad \text { on } U \cap W
$$

for a suitable function $\Phi$.

Proof of Proposition 4.1.1. Applying Lemmas 8.2.1-8.2.3, and using a partition of unity, one constructs easily an $\omega$-preferred Riemannian structure on $V$. Our proposition is proved.

8.3. Proof of Proposition 4.2.1. Suppose that an $\omega$-preferred Riemannian structure $g$ is given. In the case $\Sigma=\emptyset$, our proposition is trivial. Therefore we assume $\Sigma \neq \emptyset$. For a point $p$ of $\Sigma$ and two positive numbers $\varepsilon, \delta$, we define an open subset $U_{p}^{*}(\varepsilon, \delta)$ of $V$ containing $p$ as follows. By assumption, there exists an $\mathscr{F}$-chart $\left(U, f ; x^{1}, \cdots, x^{n+1}\right)$ at $p$ satisfying one of the following conditions:

(i) it is of type ( $\lambda$ ) for some $\lambda$, and

$$
\mathfrak{g}\left(\partial / \partial x^{i}, \partial / \partial x^{j}\right)=\delta_{i j} \quad \text { for } 1 \leq i, j \leq n ;
$$

(ii) it is of type $(*, \mu)$ for some $\mu$, and

$$
\mathfrak{g}\left(\partial / \partial x^{i}, \partial / \partial x^{j}\right)=\delta_{i j}, \quad \mathfrak{g}\left(\partial / \partial x^{i}, \partial / \partial x^{n}\right)=0 \quad \text { for } 1 \leq i, j \leq n-1 .
$$

In the case where the condition (i) is satisfied, we define $U_{p}^{*}(\varepsilon, \delta)$ to be the set of those points $z$ of $U$ satisfying the following inequalities:

(i)*

$$
\begin{gathered}
\left|-\left(x^{1}(z)\right)^{2}-\cdots-\left(x^{2}(z)\right)^{2}+\cdots+\left(x^{n}(z)\right)^{2}\right|<\varepsilon, \\
\left(\left(x^{1}(z)\right)^{2}+\cdots+\left(x^{2}(z)\right)^{2}\right)\left(\left(x^{\lambda+1}(z)\right)^{2}+\cdots+\left(x^{n}(z)\right)^{2}\right)<\varepsilon, \\
\left|x^{n+1}(z)-x^{n+1}(p)\right|<\delta .
\end{gathered}
$$

Next, in the case where the condition (ii) is satisfied, to define $U_{p}^{*}(\varepsilon, \delta)$, we consider a continuous function $G: U \rightarrow \boldsymbol{R}$ having the following properties :

(a) $G(x) \geq 0$ for $x \in U$,

(b) $G(x)=0$ for $x \in U \cap \Sigma$,

(c) $Y(G)=0$ on $U$,

(d) for any $x \in U \cap \Sigma$, the restriction $\bar{G}$ of $G$ to the subset $f^{-1}(f(x))$ $\cap S(x)$, where $S(x)$ is the connected component containing $x$, of the intersection of $U$ and the leaf through $x$, is of class $C^{1}$ except at points of $\Sigma$, and the set of critical values of $\bar{G}$ has measure zero in $\boldsymbol{R}$, 
(e) for any $x \in U \cap \Sigma$ and the map $\bar{G}$ as above, there exists a positive number $\varepsilon$ such that the set $\bar{G}^{-1}([0, \varepsilon])$ is compact.

By elementary, geometric arguments, one can verify that such a function $G$ exists. Now, in the case where (ii) is satisfied, we define $U_{p}^{*}(\varepsilon, \delta)$ to be the set of those points $z$ of $U$ satisfying the following inequalities :

$$
1-\left(x^{1}(z)\right)^{2}-\cdots-\left(x^{\mu}(z)\right)^{2}+\cdots+\left(x^{n-1}(z)\right)^{2}
$$

$$
\begin{aligned}
& \quad+g\left(x^{n}(z), x^{n+1}(z)\right)-g\left(x^{n}(p), x^{n+1}(z)\right) \mid<\varepsilon, \\
& G(z)<\varepsilon,\left|x^{n+1}(z)-x^{n+1}(p)\right|<\delta,
\end{aligned}
$$

where $g$ is the same function as in the definition of an $\mathscr{F}$-chart of "type $(*, \mu) "$ (Definition 4.1.1).

For each point $p$ of $\Sigma$, one can prove that for small $\varepsilon$, if $\delta$ is sufficiently small, the set $U_{p}^{*}(\varepsilon, \delta)$ above has the properties corresponding to the conditions (ii)-(ix) of Definition 4.2.1. Consequently, since $\Sigma$ is compact, we find a finite number of points $p_{1}, \cdots, p_{m}$ of $\Sigma$ and positive numbers $\varepsilon_{i}, \delta_{i}, i=1, \cdots, m$, such that the union

$$
T^{*}=\bigcup_{i=1}^{m} U_{p_{i}}^{*}\left(\varepsilon_{i}, \delta_{i}\right)
$$

has the required properties. Proposition 4.2.1 is proved.

\section{APPENDIX}

The generality of Condition $(\mathrm{T})$.

Our purpose is to prove:

Proposition A. For the given foliated structure $\omega$ on $V$, there exists a completely integrable one-form $\check{\omega}$ of class $C^{r}$ which satisfies (T) and is arbitrarily close to $\omega$ in the $C^{r}$ topology and is expressed as

$$
\tilde{\omega}=\tilde{a} \omega
$$

with a suitable positive-valued function a defined on $V$.

One can easily prove this proposition by the usual argument as in the proof of Milnor [4, p. 14, Theorem 2.7] if one verifies the following lemma. 
LEMMA A.1. Let $U$ be an open set in $\boldsymbol{R}^{n+1}$ with coordinates functions $\left(x^{1}, \cdots, x^{n+1}\right)$. Let $K$ be a compact subset of $U$, and $f: U \rightarrow \boldsymbol{R} a C^{s}$ function, $3 \leq s \leq \infty$. Then there exist $C^{s}$ functions $g: U \rightarrow \boldsymbol{R}$ which are arbitrarily close to $f$ in the coarse $C^{s}$ topology, such that the restriction to $K$ of $g$ satisfies the following condition:

If for some point $p$ of $K$,

$$
g_{1}(p)=\cdots=g_{n}(p)=0 \text {, and } \operatorname{det}\left(g_{i j}(p) ; i, j=1, \cdots, n\right)=0 \text {, }
$$

then

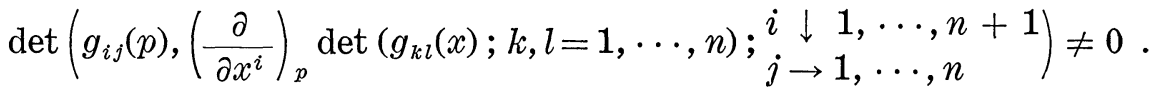

Here $g_{i}, g_{i j}$ denote $\partial g / \partial x^{i}, \partial^{2} g / \partial x^{i} \partial x^{j}$, respectively.

Proof. Consider a map $h: \boldsymbol{R}^{N} \rightarrow \boldsymbol{R}^{n+1}$ defined by

$$
h\left(y^{0}, y^{1}, \cdots, y^{n}, y^{11}, y^{12}, \cdots, y^{n n}\right)=\left(y^{1}, \cdots, y^{n}, \operatorname{det}\left(y^{i j}\right)\right) .
$$

Let $0^{n+1}$ denote the origin of $\boldsymbol{R}^{n+1}$. Then the set $P=h^{-1}\left(0^{n+1}\right)$ is a stratified set in the following way. For an integer $k, 0 \leq k \leq n$, we put

$$
\begin{aligned}
P_{k}=\left\{\left(y^{0}, y^{1}, \cdots, y^{n}, y^{11}, y^{12}, \cdots, y^{n n}\right) \in \boldsymbol{R}^{N} \mid y^{1}=\right. & \cdots=y^{n}=0, \\
& \left.\operatorname{rank}\left(y^{i j}\right) \leq n-k\right\} .
\end{aligned}
$$

Then it is easily verified that

(i ) $P=P_{1} \supset P_{2} \supset \cdots \supset P_{n}$;

(ii) for $k=1, \cdots, n-1$, the subset $P_{k}-P_{k+1}$ is a regular submanifold of $R^{N}$ with codimension $n+k^{2}$;

(iii) $P_{n}$ coincides with the submanifold $\boldsymbol{R} \times 0^{N-1}$ of $\boldsymbol{R}^{N}$. Furthermore, we see easily that $P_{2}$ coincides with the subset of $R^{N}$ where $h$ is not transversal to $0^{n+1}$, and hence that the restriction to $R^{N}-P_{2}$ of $h$, is transversal to $0^{n+1}$.

On the other hand, for a $C^{s}$ function $g: U \rightarrow R$, we define a function $J^{2}(g): U \rightarrow \boldsymbol{R}^{N}$ by the formula

$$
J^{2}(g)(x)=\left(g(x), g_{1}(x), \cdots, g_{n}(x), g_{11}(x), g_{12}(x), \cdots, g_{n n}(x)\right), \quad x \in U .
$$

By Thom's jet transversality lemma ([7], [3]), we know that the map $f$ can be approximated, in the coarse $C^{s}$ topology, as closely as desired, by a "good" map $g$ in the following sense: the restriction to $K$ of $J^{2}(g)$ is transversal to $P$. Since for $k \geq 2$, the codimension of $P_{k}-P_{k+1}$ in $\boldsymbol{R}^{N}$ is greater than $\operatorname{dim} U=n+1$, such "good" maps do not intersect 
$P_{2}$. Consequently, for the "good" map $g$, the composition $h \circ J^{2}(g)$ is transversal to the origin of $\boldsymbol{R}^{n+1}$. This fact implies that $g$ satisfies the condition $\left(\mathrm{T}^{\prime}\right)$. Lemma A.1 is proved.

\section{REFERENCES}

[1] P. Hartman, Ordinary Differential Equations, Wiley, New York, 1964.

[ 2 ] S. Lang, Introduction to Differentiable Manifolds, Interscience, New York, 1962.

[ 3 ] J. Martinet, Sur les singularités des formes différentielles, Ann. Inst. Fourier, Grenoble, 20 (1970), 95-178.

[4] J. Milnor, Lectures on the $h$-Cobordism Theorem, Princeton Univ. Press, Princeton, New Jersey, 1965.

[5] M. Morse and S. Cairns, Critical Point Theory in Global Analysis and Differential Topology, Academic Press, New York, 1969.

[6] G. Reeb, Sur certaines propriétés topologiques des variétés feuilletées, Actualités Sci. Ind. No. 1183, Hermann, Paris, 1952, 93-154.

[ 7 ] R. Thom, Un lemme sur les applications différentiables, Bol. Soc. Mat. Mexicana, 1956, 59-71.

[ 8 ] K. Yamato, Qualitative theory of codimension-one foliations, Proc. Japan Acad., 48 (1972), 356-359.

[9] K. Yamato, Codimension-one foliations with singularities (to appear).

Mathematical Institute

Nagoya University 